\title{
The Satisfiability Threshold for a Seemingly Intractable Random Constraint Satisfaction Problem
}

\author{
Harold Connamacher \\ Case Western Reserve University \\ Michael Molloy \\ University of Toronto
}

May 26, 2018

\begin{abstract}
We determine the exact threshold of satisfiability for random instances of a particular NP-complete constraint satisfaction problem (CSP). This is the first random CSP model for which we have determined a precise linear satisfiability threshold, and for which random instances with density near that threshold appear to be computationally difficult. More formally, it is the first random CSP model for which the satisfiability threshold is known and which shares the following characteristics with random $k$ SAT for $k \geq 3$. The problem is NP-complete, the satisfiability threshold occurs when there is a linear number of clauses, and a uniformly random instance with a linear number of clauses asymptotically almost surely has exponential resolution complexity.
\end{abstract}

\section{Introduction}

Determining the satisfiability threshold for random $k$-SAT is a fundamental problem that has received attention from several scientific communities. (See, eg., 1 for a survey of the area.) The basic question is this: does there exist a constant $c_{k}^{*}$ such that a uniformly random instance of $k$-SAT with $n$ variables and $c n$ clauses will be asymptotically almost surely (a.a.s.) 1] satisfiable if $c<c_{k}^{*}$ and a.a.s. unsatisfiable if $c>c_{k}^{*}$ ? Neither the existence nor the location of $c_{k}^{*}$ is known for any $k \geq 3$; Friedgut 32 proved that the threshold for $k$-SAT is sharp, although the location of the threshold might be not be at the same clause density for each value of $n$. We have a tight asymptotic bound on the conjectured $c_{k}^{*}$ : $2^{k} \log 2-\mathrm{O}(k)\left[\underline{6} \leq c_{k}^{*} \leq 2^{k} \log 2\right.$ 31. For 3-SAT, the current state of research has 3.52 [37, 45] $\leq c_{3}^{*} \leq 4.4898$ [25]. From experimental evidence, the threshold for 3 -SAT appears to be roughly 4.2 [47, 69, 20].

\footnotetext{
${ }^{1}$ A property holds asymptotically almost surely if its probability tends to 1 as the number of variables tends to $\infty$.
} 
Research on the satisfiability threshold has extended to generalizations of $k$-SAT such as the Schaefer [68] generalizations: 1-in- $k$ SAT [3] (only one true literal per clause), NAE-SAT [3, 5] (at least one true and one false literal per clause), and XOR-SAT [27, 52, 26] (each clause is an exclusive-or rather than a disjunction), and more generally, to random constraint satisfaction problems.

In a constraint satisfaction problem (CSP), we can allow variables to take on values from a domain of size larger than 2 , and we have more freedom as to the types of constraints to use. There have been various models proposed and studied for random constraint satisfaction problems, eg. 4, 21, 53, 56, 58]. For such models, a major goal has typically been to determine whether it has a sharp satisfiability threshold, and if so, to determine its location. In addition, there has been a large body of experimental studies 35 to find the approximate location of the satisfiability threshold and to study the difficulty of solving random instances of various CSP models.

The primary motivation for a large number of these studies was the discovery by Selman, et al 69. that random instances of 3-SAT with clause density near the conjectured threshold are very difficult to solve. Researchers who develop SAT-solvers study such problems to test and improve their solvers and to look for insights into what can make SAT instances computationally difficult. When these researchers expanded to CSP-solvers, they wanted to find thresholds for other random CSP models with the expectation that this should provide a rich source of difficult instances.

Most of this research has been on models where the constraint- and domainsizes are constant. There have been studies of models where one or both of these parameters grows with $n$ (eg. [29, 72, 73, 33, 30]). As discussed below, such models tend to have a very different nature; eg., the thresholds typically occur when the number of constraints is superlinear. So our focus in this discussion is on the case where these parameters are both constant.

Most random CSP models for which the satisfiability threshold is known are in P (eg. 2-SAT [15, 24, 36, 3-XOR-SAT 27, 26]), and hence do not provide difficult instances. We know the satisfiability threshold for a few NP-complete problems with constant sized domain and constraints, for example 1-in- $k$-SAT [3], and a mixture of 2-SAT and 3-SAT when the number of clauses of size 3 is kept small [60, and a model from [59. However, in each of these cases, a random instance whose number of clauses is just below the threshold, is in some sense equivalent to random 2-SAT. This allows us to prove that such problems are a.a.s. satisfiable by analyzing a polynomial time algorithm that solves them with uniformly positive probability (w.u.p.p.) 2. Furthermore, instances with density slightly above the threshold a.a.s. have short resolution proofs of unsatisfiability. (This is straightforward to show for 1 -in- $k$-SAT, and is proven in 2, 59 for the other two.) It is straightforward to design polynomial time algorithms that make use of such proofs to a.a.s. recognize that such instances are unsatisfiable. Thus, while these problems are NP-hard, they are easy to solve

\footnotetext{
${ }^{2} \mathrm{~A}$ property holds with uniformly positive probability if as the number of variables tends to $\infty$, the liminf of its probability is at least some positive constant.
} 
when their density is near the satisfiability threshold.

In this paper, we introduce a particular NP-hard problem and determine its satisfiability threshold. The problem is different from previous NP-hard problems whose thresholds are known in that we don't know of any algorithm that can solve random instances whose density is near the threshold. Furthermore, we can prove that such instances a.a.s. have exponential resolution complexity, which implies that resolution based algorithms will a.a.s. fail on unsatisfiable instances. (Virtually all complet 3 CSP-solvers that are commonly used in practice are resolution-based.)

This is the first random constraint satisfaction problem for which: (1) We have determined a constant $c^{*}$ such that if the number of constraints is at most $\left(c^{*}-\epsilon\right) n$ then it is a.a.s. satisfiable and if the number of constraints is at least $\left(c^{*}+\epsilon\right) n$ then it is a.a.s. unsatisfiable. (2) We don't know of any polynomial time algorithm that will a.a.s. succeed on a random instance when the number of clauses is $c n$ for $c$ arbitrarily close to $c^{*}$. In short, we know the location of a satisfiability threshold around which the instances actually appear to be difficult.

Our problem is called $(3,4)$-UE-CSP. We will define $(k, d)$-UE-CSP in the next section. We use $U_{n, m}^{(k, d)}$ to denote a random instance with $n$ variables and $m$ constraints. In Subsection 5.1 we will specify a precise constant $c^{*}=.917935 \ldots$ Our main theorem is:

Theorem 1 If $c<c^{*}$ then $U_{n, m=c n}^{(3,4)}$ is a.a.s. satisfiable. If $c>c^{*}$ then $U_{n, m=c n}^{(3,4)}$ is a.a.s. unsatisfiable.

In 19 the first author proves

Theorem 2 For every $d \geq 4,(3, d)$-UE-CSP is NP-complete. Furthermore, it is NP-complete under the restriction that no two constraints share more than one variable.

The latter restriction is relevant here because random instances with a linear number of constraints a.a.s. have that property.

In Section 4 we prove

Theorem 3 For any constant $c>0$, and any $k \geq 3, d \geq 2$, the resolution complexity of $U_{n, m=c n}^{(k, d)}$ is a.a.s. $2^{\Theta(n)}$.

The proof of Theorem 1 follows along the lines of that of Dubois and Mandler 27, 28, who determined the satisfiability threshold for $k$-XOR-SAT 4 That problem is in $\mathrm{P}$ - it can be solved using Gaussian elimination. However, they did not make use of the fact that it is in $\mathrm{P}$ in establishing the threshold. Rather, they applied the second moment method to prove that problems with density

\footnotetext{
${ }^{3}$ A solver is complete if it is able to recognize all satisfiable and all unsatisfiable instances.

${ }^{4}$ Unfortunately, a full version of their paper never appeared, and the short versions provide only a sketch of the argument for $k=3$ and no details for $k=4$. Ditzfelbinger et. al. 26] provide a full proof for all $k \geq 3$, using a somewhat different technique.
} 
below the threshold are a.a.s. satisfiable. The problem $(3,4)$-UE-CSP is somewhat contrived in that it is designed to have the properties of $k$-XOR-SAT that allow the approach of Dubois and Mandler to work, while at the same time being NP-complete. The analysis is much more complicated than that of [27, 28. In fact, we have to resort to a computer-aided proof which makes use of interval analysis to rigorously determine the global maximum of a particular function.

The discussion above has focused on random CSP models in which the constraint size and domain size are both constant. Researchers have studied models where the domain size grows with $n[29,34,70,72,173]$ or where the constraint size grows with $n$ [33, 30. Exact thresholds are known for some such models [72, 73, 33, 30]. In [73] it is proven that the models of [72] and [73] contain many problems that a.a.s. have exponential tree resolution complexity, and it is noted that the model of 33 contains problems that a.a.s. have exponential resolution complexity. But in all of these models, the satisfiability threshold occurs when the number of clauses is superlinear in $n$, and the structure of a random constraint satisfaction problem with a superlinear number of clauses is very different from one with a linear number of clauses. Thus, these problems are of a very different nature than, say, the random 3-SAT problem.

In summary, random $(3,4)$-UE-CSP is the first model of random constraint satisfaction problems for which we know the exact satisfiability threshold and which shares the following characteristics with random 3-SAT: (a) it is NPcomplete; (b) there are a linear number of constraints at the threshold; (c) random instances near the threshold a.a.s. have exponential resolution complexity; (d) the domain and constraint sizes are constant. Random 3-XOR-SAT was close - it met all of these conditions except for (a).

Ours is also the first random CSP model with a known linear satisfiability threshold for which it is seems that random instances with density near that threshold are difficult to solve. There is computational evidence that such problems really are difficult. Since the appearance of a short preliminary version of this paper [18, random instances of $(3,4)$-UE-CSP with densities close to the satisfiability threshold have been chosen as challenging problems in experimental studies [49, 40, 41, 42, 43. In fact, the study of [7] indicated that the problems become difficult when the density exceeds the threshold for the appearance of a 2-core in the underlying hypergraph (see Section 2). Random instances of $(3,4)$-UE-CSP have also been used as test cases for the SAT solver competitions of the SAT 2004, 2005, 2007, and 2009 conferences [11, 65, 66, 67. None of the solvers in the competition were able to solve a test instance on 1200 variables whose density was at the satisfiability threshold.

Remark: A short preliminary version of this paper appeared in a conference proceedings [18. There is an error in that version; specifically in the proof of Lemma 16, which corresponds to Lemma 18 in this paper. We replaced that proof with the much longer computer-aided proof found here. 


\section{Uniquely Extendible CSPs}

We define a clause to be an ordered subset of variables and a constraint to be a list of tuples of values that we may assign to the variables of the clause. A constraint satisfaction problem consists of a set of $n$ variables where each variable has a non-empty domain of possible values, a set of $m$ clauses, and a constraint applied to each clause. The goal is to find an assignment to the variables such that the constraint on each clause is satisfied. One common restriction is that every variable must have the same domain of values; that will be the case throughout this paper. In keeping with SAT notation, we will denote an instance of a constraint satisfaction problem as a formula. The underlying hypergraph of a CSP is the hypergraph whose vertices are the variables and whose hyperedges are the clauses.

The inspiration for uniquely extendible CSPs comes from the proof of the satisfiability threshold for 3-XOR-SAT [27, 52, 26]. In XOR-SAT, each clause is an exclusive-or of the literals, rather than a disjunction. Thus each clause can be interpreted as a linear equation mod 2, which is why it can be solved using Gaussian elimination.

As we mentioned above, their proof of the threshold does not rely in any way on the Gaussian elimination algorithm. Instead, they reduce the random formula to its 2-core, the unique maximal subformula where each variable occurs in at least two clauses. Then, the first and second moment methods are used to give coinciding upper and lower bounds on the satisfiability threshold for the 2-core. Standard calculations translate the satisfiability threshold for the 2-core into a satisfiability threshold for 3-XOR-SAT.

The key property that makes this proof work is that every constraint in XOR-SAT is uniquely extendible:

Definition 4 A constraint on $k$-variables is uniquely extendible if for every truth assignment to any $k-1$ of its variables there is exactly one value that can be assigned to the remaining variable so that the constraint will be satisfied. It is at-most-one-extendible if there is always at most one such value and at-leastone-extendible if there is always at least one such value.

It is the property that the constraints of $k$-XOR-SAT are at-least-one-extendible that permits us to consider only the 2-core. Consider the following procedure to find the 2-core:

CORE: While the formula has any variable that occurs in at most one clause, choose an arbitrary such variable and delete it along with any clause that contains it.

The order in which variables are chosen to be deleted is easily seen to be irrelevant in that it does not affect the final output of the procedure. This proves that the 2-core is unique.

Lemma 5 Let $F$ be an instance of a CSP such that every constraint is at-leastone-extendible. Then $F$ is satisfiable iff the 2-core of $F$ is satisfiable. 
Proof Clearly, if the 2-core of $F$ is unsatisfiable then so is $F$. Assume that the 2-core of $F$ is satisfiable. Consider running CORE on $F$, and suppose that the deleted variables are $x_{1}, x_{2}, \ldots, x_{t}$ in that order. Start with any satisfying assignment of the 2-core. Now restore the deleted variables in reverse order, i.e. $x_{t}, x_{t-1}, \ldots, x_{1}$, each time adding the variable along with the at most one clause that was deleted when the variable was deleted. Because the constraint applied to the clause is at-least-one-extendible, there is a value that can be assigned to the variable that does not violate the constraint. This will result in a satisfying assignment for $F$.

The fact that the constraints are all at-most-one-extendible is crucial to the fact that the first moment bound yields the exact satisfiability threshold for the 2-core of a random $k$-XOR-SAT formula. To be more specific: let $X$ denote the number of satisfying assignments. A straightforward calculation shows that if the number of clauses is $c n$ then $\mathbf{E}(X)=2^{f(c) n+o(n)}$, where $f(c)$ is a continuous monotonically decreasing function; in fact, this is true for virtually all models of random CSP's where the domain size is constant (of course, $f(c)$ depends on the model). We define $c^{+}$to be the solution of $f(c)=0$ and note that if $c>c^{+}$then $\mathbf{E}(X)=2^{-\Theta(n)}$ and so the problem is a.a.s. not satisfiable. For most problems, $c^{+}$is strictly greater than the actual satisfiability threshold. Usually this can be seen by observing a "jackpot phenomena" where the presence of at least one satisfying assignment a.a.s. implies the presence of an exponential number of them.

This is most easily seen in the random instance before it is stripped to the 2-core. Standard arguments show that such an instance will a.a.s. have at least $\epsilon(c) n$ variables of degree zero, for some $\epsilon(c)>0$. Clearly, any satisfying assignment remains satisfying after changing the values of any of those variables. So a.a.s. $X>0$ implies $X \geq 2^{\epsilon(c) n}$. By continuity, we can choose some $c<c^{+}$ such that $\epsilon(c)>2 f(c)>0$. Markov's Inequality implies that $\operatorname{Pr}\left(X \geq 2^{\epsilon(c) n}\right)<$ $\mathbf{E}(X) / 2^{\epsilon(c) n}=2^{f(c) n+o(n)-\epsilon(c) n}<2^{-f(c) n}$. Therefore a.a.s. $X<2^{\epsilon(c) n}$, and so a.a.s. $X=0$; i.e. the formula is a.a.s. unsatisfiable. This implies that the satisfiability threshold (if it exists) is at most $c<c^{+}$.

Stripping to the 1-core will eliminate this particular jackpot, but a similar jackpot will remain. There will a.a.s. be a linear number of clauses with two variables that don't appear in any other clauses. For any satisfying assignment, there will always be a way to jointly change the values of those two variables so that the assignment is still satisfiable. So once again we a.a.s. have $X>0$ implies $X \geq 2^{\Theta(n)}$.

But if we strip to the 2-core then these, and all similar jackpots, are eliminated. To see this, consider any satisfying assignment. If we change the assignment of any one variable, then the constraint on every clause it is in will become violated because they are all at-most-one-extendible. So we must change the values of at least one other variable in each of those clauses. Because every variable lies in at least 2 clauses (as we are in the 2-core) each such change will cause the constraint on another clause to be violated. Expansion properties of random formulas ensure that this pattern spreads until many variables must be changed - far too many to create a jackpot of the type described above. Of 
course, this doesn't imply that $c^{+}$will be the actual satisfiability threshold, but it shows that a common simple argument fails to prove that it is not.

Observing that unique satisfiability played a crucial role in this proof, we are inspired to define a more general class of problems which may also be amenable to similar analysis.

Definition 6 (UE-CSP) A UE-CSP instance is a constraint satisfaction problem where every constraint is uniquely extendible.

Definition $7((k, d)$-UE-CSP) An instance of $(k, d)-U E-C S P$ is an instance of UE-CSP where we restrict every clause to have size $k$ and every variable to have domain $\{1, \ldots, d\}$.

A straightforward induction on $k$ yields that there are only two different uniquely extendible constraints of size $k$ with $d=2$, and that they are equivalent to the two different possible parities for a $k$-XOR-SAT constraint. In other words, $(k, 2)$-UE-CSP is exactly $k$-XOR-SAT, and thus is in P.

In addition, both $(k, 3)$-UE-CSP and $(2, d)$-UE-CSP are in $\mathrm{P}$ [19. On the other hand, Theorem 2 states that $(3, d)$-UE-CSP is NP-complete for any $d \geq$ 4 [19]. In this paper, we focus on the simplest NP-complete case: $(3,4)$-UE-CSP.

\section{The Random Model}

For each appropriate $n, m$, we define $\Omega_{n, m}^{(k, d)}$ to be the set of $(k, d)$-UE-CSP instances with $m$ clauses on variables $\left\{v_{1}, \ldots, v_{n}\right\}$ and a uniquely extendible constraint on each clause. We define $U_{n, m}^{(k, d)}$ to be a uniformly random member of $\Omega_{n, m}^{(k, d)}$. When $m$ is defined to be some function $g(n)$, we often write $U_{n, m=g(n)}^{(k, d)}$. As is common in the study of random problems of this sort, we will be most interested in the case where $m=c n$ for some constant $c$. This model is equivalent to first choosing a uniformly random hypergraph on $n$ vertices and $m$ hyperedges to be the underlying hypergraph of the $(k, d)$-UE-CSP instance, and then for each hyperedge, arbitrarily ordering the vertices of the hyperedge and choosing a uniformly random uniquely extendible constraint of size $k$ and domain size $d$.

Alternatively, we can consider a second random model. Define $U_{n, p}^{(k, d)}$ to be an instance of $(k, d)$-UE-CSP on $n$ variables where each of the $\left(\begin{array}{l}n \\ k\end{array}\right)$ clauses occurs in $U_{n, p}^{(k, d)}$ with probability $p$ and a uniformly random constraint is applied to each clause.

From results of [12, 50, on random structures, the two models are asymptotically equivalent in the sense that

$$
\lim _{n \rightarrow \infty} \operatorname{Pr}\left(U_{n, m}^{(k, d)} \text { has property } \mathcal{A}\right)=\lim _{n \rightarrow \infty} \operatorname{Pr}\left(U_{n, p}^{(k, d)} \text { has property } \mathcal{A}\right)
$$

if $\mathcal{A}$ is a monotone (increasing or decreasing) property and $m$ and $\left(\begin{array}{l}n \\ k\end{array}\right) p$ are 
"close" to each other. Formally, $m$ is "close" to $\left(\begin{array}{l}n \\ k\end{array}\right) p$ if

$$
m=\left(\begin{array}{l}
n \\
k
\end{array}\right) p+\mathrm{O}\left(\sqrt{\left(\begin{array}{l}
n \\
k
\end{array}\right) p(1-p)}\right),
$$

and $p$ is "close" to $\frac{m}{\left(\begin{array}{l}n \\ k\end{array}\right)}$ if

$$
p=\frac{m}{\left(\begin{array}{l}
n \\
k
\end{array}\right)}+\mathrm{O}\left(\sqrt{\frac{m\left(\left(\begin{array}{l}
n \\
k
\end{array}\right)-m\right)}{\left(\begin{array}{l}
n \\
k
\end{array}\right)^{3}}}\right) .
$$

So in particular, Theorem 1 implies that $U_{n, p=d / n^{2}}^{(3,4)}$ has a sharp threshold of satisfiability at $d^{*}=6 c^{*}$.

We need another random model with which to analyze the 2-core of $U_{n, m}^{(3,4)}$. Let $\Psi_{n, m}$ denote the subset of $\Omega_{n, m}^{(3,4)}$ in which every variable lies in at least 2 clauses, and let $U_{n, m}^{*}$ denote a uniformly random member of $\Psi_{n, m}$. The following lemma allows us to work in the $U_{n, m}^{*}$ model.

Lemma 8 For any $n, m, n^{\prime}, m^{\prime}$, if we condition on the event that the 2-core of $U_{n, m=c n}^{(3,4)}$ has $n^{\prime}$ variables and $m^{\prime}$ clauses, then that 2-core is a uniformly random member of $\Psi_{n^{\prime}, m^{\prime}}$.

Proof This is a straightforward variation of the proof of Claim 1 in the proof of Lemma 4(b) from [57, which is itself a very standard argument. Consider a formula $F$ and its 2-core $F_{c}$. Assume $F_{c}$ has $n^{\prime}$ variables and $m^{\prime}$ clauses. Replace $F_{c}$ in $F$ by a arbitrary member of $\Psi_{n^{\prime}, m^{\prime}}, F_{c}^{\prime}$. Call this new formula $F^{\prime}$. Note that $F_{c}^{\prime}$ is the 2-core of $F^{\prime}$ and that $F^{\prime}$ and $F$ have the same number of clauses. Thus, the probability a random formula is equal to $F$ is the same as the probability it is equal to $F^{\prime}$, and this implies the probability that the 2-core of a random formula is $F_{c}$ is equal to the probability that the 2-core is $F_{c}^{\prime}$.

\section{Resolution Complexity}

While the uncertainty of the $\mathrm{P}$ versus NP question means that we cannot state definitively whether an efficient algorithm exists to either solve an instance of $(k, d)$-UE-CSP or prove no solution to the instance exists, we can state that there is no efficient resolution based algorithm, such as DPLL, that will correctly handle unsatisfiable instances of $(k, d)$-UE-CSP for $k \geq 3$.

The vast majority of SAT- and CSP-solving algorithms that are commonly used in practice will recognize an unsatisfiable problem by implicitly producing a resolution proof that it is unsatisfiable (see [53] for the definition of resolution proof). The resolution complexity of a boolean formula is the length of the shortest resolution proof that it is unsatisfiable. (If the formula is satisfiable then the resolution complexity is $\infty$.) Thus, the resolution complexity of an unsatisfiable 
formula is a lower bound on the time that it will take an algorithm to solve the problem. In a seminal paper, Chvátal and Szemerédi[16] proved that a random instance of 3-SAT with a linear number of clauses a.a.s. has exponentially high resolution complexity. This partially explains the aforementioned observation of Selman et al[69 that SAT-solving algorithms take a very long time on random instances whose density is near the satisfiability threshold.

Mitchell[54 discusses two natural ways to extend the notion of resolution complexity to the setting of a CSP. These two measures of resolution complexity are denoted $\mathbf{C}-\mathbf{R E S}$ and $\mathbf{N G}$ - RES. The latter appears on the surface to be the most natural extension in that it extends resolution rules to the setting of a CSP and then carries them out. C - RES, on the other hand, converts a CSP to a boolean CNF-formula and then carries out CNFresolution on that formula. Mitchell shows that for every CSP instance $\mathcal{I}$, $\mathbf{C}-\mathbf{R E S}(\mathcal{I}) \leq \operatorname{poly}(\mathbf{N G}-\mathbf{R E S}(\mathcal{I}))$ whereas there are many choices for $\mathcal{I}$ for which the converse it not true. Furthermore, all commonly used resolution-type CSP algorithms correspond nicely to the $\mathbf{C}-\mathbf{R E S}$ complexity of the input, but there are some that do not correspond to the NG-RES. For that reason, we focus in this paper on the $\mathbf{C}-\mathbf{R E S}$ complexity, as did Mitchell in [53. But note that the above inequality implies that Theorem 3, which we restate below, also holds for the NG - RES complexity.

Theorem 3 For any constant $c>0$, and any $k \geq 3, d \geq 2$, the $\mathbf{C}-\mathbf{R E S}$ resolution complexity of a uniformly random instance of $(k, d)$-UE-CSP with $n$ variables and cn clauses is a.a.s. $2^{\Theta(n)}$.

Proof From techniques developed in 9, 53, 59, 53, a.a.s. the shortest C - RES resolution proof of unsatisfiability of a constraint satisfaction problem on $n$ variables has exponential size if there exists constants $\alpha, \zeta>0$ such that a.a.s. the following three conditions hold.

1. Every subformula on at most $\alpha n$ variables is satisfiable.

2. Every subproblem on $v$ variables, where $\frac{1}{2} \alpha n \leq v \leq \alpha n$, has at least $\zeta n$ variables of degree at most 1 , where the degree of a variable is the number of clauses containing that variable.

3. If $x$ is a variable of degree at most 1 in a CSP $F$ then, letting $F^{\prime}$ be the subproblem obtained by removing $x$ and its clause, every satisfying assignment of $F^{\prime}$ can be extended to a satisfying assignment of $F$ by assigning some value to $x$.

Because our random model for UE-CSP applies one uniquely extendible constraint to each clause, the third condition is trivially true. The following lemma from [59] states a useful property of random formulae with a linear number of clauses: a.a.s. every subproblem on at most $\alpha n$ variables has a low clause density. A similar lemma is proven in 53 and several other papers.

Lemma 9 ([59]) Let $c>0$ and $k \geq 2$ and let $H$ be a random $k$-uniform hypergraph with $n$ vertices and $m=$ cn edges. Then for any $\delta>0$, there exists 
$\alpha=\alpha(c, k, \delta)>0$ such that a.a.s. $H$ has no subgraph with $0<h \leq\lfloor\alpha n\rfloor$ vertices and at least $\left(\frac{1+\delta}{k-1}\right) h$ edges.

Let $F^{\prime}$ be a minimally unsatisfiable subformula of $F$ with at most $h \leq\lfloor\alpha n\rfloor$ variables. Then $F^{\prime}$ cannot have a variable of degree less than 2 because condition 3 above is trivially true for UE-CSP. Thus, $F^{\prime}$ must have at least $\left(\frac{2}{k}\right) h$ edges. However, if $k>2$, there exists $\delta=\delta(k)>0$ such that $\frac{2}{k}>\frac{1+\delta}{k-1}$. As a result, a.a.s. every subformula on $h$ variables, and in particular $F^{\prime}$, will be satisfiable. By the same argument, let $\zeta=1-\frac{(1+\delta) k}{2(k-1)}$, and $F^{\prime}$ must have at least $\zeta n$ variables of degree at most 1 .

\section{The Satisfiability Threshold for $(3,4)$-UE-CSP}

In this section we formally define $c^{*}$ and prove Theorem 1, our main theorem.

The proof will proceed as follows: first the variables of degree 0 and 1 are stripped away to produce the 2-core. Then the satisfiability threshold on the 2 -core is proven using the first and second moment methods, and this threshold on the 2-core yields the threshold for the original problem. The upper bound for satisfiability is a simple use of the first moment method, but the lower bound is more challenging. Applying the second moment method produces a complicated summation. Following the example of [27, the summation is approximated by a multiple integral, and then the Laplace Method is used to approximate the integral. In order to apply the Laplace Method, we must determine the global maximum of a certain function (see Lemma 18). We do so with a computer aided proof using interval analysis, which we present in the appendix.

\subsection{Determining $c^{*}$}

Since we will be working with the 2-core, we need to determine how its density relates to that of the original formula.

Lemma 10 Let $U_{n, m=c n}^{(3,4)}$ be a uniformly random instance of $(3,4)-U E$-CSP with $n$ variables and $m=c n$ clauses. If $c<\min _{x>0} \frac{x}{3\left(1-\mathrm{e}^{-x}\right)^{2}}=0.818469 \ldots$, a.a.s. $U_{n, m=c n}^{(3,4)}$ has no non-empty 2-core. Otherwise, a.a.s. the 2-core of $U_{n, m=c n}^{(3,4)}$ has $\Theta(n)$ variables and $\gamma(c)+\mathrm{o}(1)$ times as many clauses as variables with

$$
\gamma(c)=\frac{x\left(1-\mathrm{e}^{-x}\right)}{3\left(1-\mathrm{e}^{-x}-x \mathrm{e}^{-x}\right)},
$$

where $x$ is the largest solution to

$$
x=3 c\left(1-\mathrm{e}^{-x}\right)^{2} .
$$

Proof Recall the CORE procedure from Section 2 Note that it essentially works on the underlying hypergraph of the random instance. Cores 
of random uniform hypergraphs are well-studied (see, for example, 63, 51, 57, 13, 46, 64, 22). From [13, the 2-core of a $k$-uniform random hypergraph on $n$ vertices and $c n$ edges has a.a.s. $\left(1-\mathrm{e}^{-x}-x \mathrm{e}^{-x}\right) n+\mathrm{o}(n)$ vertices

and a.a.s. $\left(1-\mathrm{e}^{-x}\right)^{k} c n+\mathrm{o}(n)$ hyperedges where $x$ is the largest solution to $x=c k\left(1-\mathrm{e}^{-x}\right)^{k-1}$, and if there is no positive solution to $x=c k\left(1-\mathrm{e}^{-x}\right)^{k-1}$ then the 2 -core is a.a.s. empty. Setting $k=3$ and rearranging completes the proof.

Below, we will prove that the satisfiability threshold for the 2-core of random $(3,4)$-UE-CSP is at clause density 1 . Thus we define:

Definition $11 c^{*}=.917935 \ldots$ is defined to be the unique solution to $\gamma(c)=1$.

\subsection{The First and Second Moment Arguments}

Here we prove that $c=1$ is the satisfiability threshold for $U_{n, m=c n}^{*}$; i.e. random $(3,4)$-UE-CSP with minimum degree at least 2 . Lemmas 8 and 10 then imply Theorem 1. We need to prove two sides of the threshold:

Lemma 12 For every $c>1, U_{n, m=c n}^{*}$ is a.a.s. unsatisfiable.

Lemma 13 For every $c<1, U_{n, m=c n}^{*}$ is a.a.s. satisfiable.

Theorem 1 then follows:

Proof of Theorem 1 Let $F$ be a random instance of $U_{n, m=c n}^{(3,4)}$. Expose $n^{\prime}, m^{\prime}$, the number of variables and constraints in the 2-core of $F$. By Lemma 10, a.a.s. $m^{\prime}=(\gamma(c)+o(1)) n^{\prime}$. By Lemma 8 , the 2-core of $F$, after conditioning on the values of $n^{\prime}, m^{\prime}$, has the same distribution as $U_{n^{\prime}, m^{\prime}}^{*}$. Therefore, the theorem follows from Lemmas 12/13 and the definition of $c^{*}$.

The proof of the first lemma is straightforward:

Proof of Lemma 12 We apply what is, in this field, a very standard first moment argument. Consider a random instance $F$ chosen from $\Psi_{n, m=c n}$, and let $N$ denote the number of satisfying assignments of $F$. We will show that $\mathbf{E}(N)=\mathrm{o}(1)$; this implies that a.a.s. $N=0$; i.e., that a.a.s. $F$ is unsatisfiable.

Consider any assignment $\sigma$ of values to the variables of $F$. Since each constraint is uniquely extendible, for each possible setting of two variables in a clause, there is exactly one possible value for the third variable. Because the random model considered includes all possible uniquely extendible constraints and because there are 4 possible values for the third variable, the probability that a particular constraint is satisfied by $\sigma$ is $\frac{1}{4}$. As there are $4^{n}$ choices for $\sigma$, we have

$$
\mathbf{E}(N)=4^{n} 4^{-m}=4^{(1-c) n}=\mathrm{o}(1)
$$

since $c>1$.

The next proof is much more complicated, and takes up most of the work in this paper. As mentioned above, we follow the proof of the corresponding theorem in 27, applying a second moment argument. Unfortunately, our larger 
domain size yields a larger set of constraints to choose from and much more complicated calculations than those in [27.

Proof of Lemma 13 As in the proof of Lemma 12, consider a random instance $F$ chosen from $\Psi_{n, m=c n}$, and let $N$ denote the number of satisfying assignments of $F$. Again, we have $\mathbf{E}(N)=4^{(1-c) n}$. The main step of this proof is to compute the second moment of $N$ obtaining:

$$
\mathbf{E}\left(N^{2}\right)=\mathbf{E}(N)^{2}(1+\mathrm{o}(1)) .
$$

Because $N$ is non-negative, a well known application of the Cauchy-Swartz inequality implies

$$
\operatorname{Pr}(N>0) \geq \frac{\mathbf{E}(N)^{2}}{\mathbf{E}\left(N^{2}\right)},
$$

and so (11) implies Lemma 13

Following the technique of [27, the proof will compute $\mathbf{E}\left(N^{2}\right)$ by putting $\frac{\mathbf{E}\left(N^{2}\right)}{\mathbf{E}(N)^{2}}$ into the form

$$
\int_{a_{1}}^{b_{1}} \int_{a_{2}}^{b_{2}} \int_{a_{3}}^{b_{3}} g\left(x_{1}, x_{2}, x_{3}\right) e^{n h\left(x_{1}, x_{2}, x_{3}\right)} d x_{1} d x_{2} d x_{3}
$$

where $g$ is polynomial in $n$ and $h$ has a unique maximum in the range of the integrals. Then we use the Laplace Method to approximate the triple integral.

Let the $4^{n}$ possible assignments be $\sigma_{1}, \ldots, \sigma_{4^{n}}$, and let $N_{i}$ be the indicator variable that $\sigma_{i}$ is a satisfying assignment. Then $N=N_{1}+\cdots+N_{4^{n}}$ and $N^{2}=\sum_{i, j} N_{i} N_{j}$. Since $N_{i} N_{j}=1$ if and only if $F$ is satisfied by both $\sigma_{i}$ and $\sigma_{j}$, this indicates that we must focus on counting the number of instances satisfied by two assignments to the variables.

Similarly to 27, let $\sigma$ and $\tau$ be arbitrary assignments to the variables, let $\# \mathcal{C}$ be the total number of instances in $\Psi_{n, m}$, and let $\# \mathcal{C}_{\sigma, \tau}$ be the total number of instances in $\Psi_{n, m}$ that are satisfied by both $\sigma$ and $\tau$. Then,

$$
\mathbf{E}\left(N^{2}\right)=\frac{1}{\# \mathcal{C}} \sum_{\sigma, \tau} \# \mathcal{C}_{\sigma, \tau} .
$$

Let $q$ be the number of different uniquely extendible constraints on three ordered variables. So for each clause, we will have $q$ choices for the constraint that we assign to that clause. As is done in [27, we can think of the $m$ clauses as inducing a distribution of $3 \mathrm{~m}$ "places" to the $n$ variables such that each variable receives at least 2 "places". In addition, we add the restriction that for each triple of "places" corresponding to a clause, no two "places" are assigned to the same variable. We define:

- $S(i, j, 2)$, known as a generalized Stirling number of the second kind, is the number of ways to partition $i$ elements into $j$ sets such that each set has at least 2 elements. 
- $\Lambda_{3 m, n}$ is the probability that, in the above partition, no two elements from any one triple appear in the same set.

So, $\# \mathcal{C}=q^{m} S(3 m, n, 2) n ! \Lambda_{3 m, n}$.

Consider a clause and a random constraint on that clause. We need to determine the probability that both assignments $\sigma$ and $\tau$ satisfy the constraint. Place an arbitrary ordering on the variables of the clause, and let $\alpha, \beta$, and $\gamma$ be the values assigned to those variables by $\sigma$, and let $\alpha^{\prime}, \beta^{\prime}$, and $\gamma^{\prime}$ be the values assigned to those variables by $\tau$. In addition, let $z_{\alpha \beta}$ be the unique value that the constraint forces the third variable to be if the first variable is assigned $\alpha$ and the second variable is assigned $\beta$.

If the values of the three variables are unchanged between $\sigma$ and $\tau$, i.e. if $\alpha=\alpha^{\prime}, \beta=\beta^{\prime}$, and $\gamma=\gamma^{\prime}$, then the probability that both assignments satisfy a random constraint is the same as the probability that a random constraint assigns the third variable $\gamma$ if the first two are assigned $\alpha$ and $\beta$ respectively. Every constraint of size 3 will permit a tuple of the form $\left(\alpha, \beta, z_{\alpha \beta}\right)$, and there are 4 possible choices for $z_{\alpha \beta}$. Exactly $\frac{1}{4}$ of the constraints will have $z_{\alpha \beta}=\gamma$. As a result,

$$
\operatorname{Pr}\left(z_{\alpha \beta}=\gamma\right)=\frac{1}{4}
$$

Thus, both assignments will satisfy a proportion of $\frac{1}{4}$ of the possible constraints.

Note that the uniquely extendible property means that if exactly one of the clause's variables changes value between $\sigma$ and $\tau$, for example if $\alpha=\alpha^{\prime}, \beta=\beta^{\prime}$, and $\gamma \neq \gamma^{\prime}$, then the constraint cannot be satisfied by both $\sigma$ and $\tau$.

Suppose one variable, assume w.l.o.g. the first variable, is assigned the same value by $\sigma$ and $\tau$ and each of the other two variables is assigned a different value in $\tau$ from what it is assigned in $\sigma$, i.e. $\alpha=\alpha^{\prime}, \beta \neq \beta^{\prime}$, and $\gamma \neq \gamma^{\prime}$. In this case, we need to determine

$$
\operatorname{Pr}\left(z_{\alpha \beta}=\gamma \wedge z_{\alpha \beta^{\prime}}=\gamma^{\prime}\right)=\operatorname{Pr}\left(z_{\alpha \beta^{\prime}}=\gamma^{\prime} \mid z_{\alpha \beta}=\gamma\right) \operatorname{Pr}\left(z_{\alpha \beta}=\gamma\right) .
$$

Every constraint of size 3 that permits the tuple $(\alpha, \beta, \gamma)$ will also permit a tuple of the form $\left(\alpha, \beta^{\prime}, z_{\alpha \beta^{\prime}}\right)$ with $z_{\alpha \beta^{\prime}} \neq \gamma$. There are 3 choices for $z_{\alpha \beta^{\prime}}$, and by symmetry, each is equally likely. So exactly $\frac{1}{3}$ of the constraints will have $z_{\alpha \beta^{\prime}}=\gamma^{\prime}$. Thus,

$$
\operatorname{Pr}\left(z_{\alpha \beta^{\prime}}=\gamma^{\prime} \mid z_{\alpha \beta}=\gamma\right) \operatorname{Pr}\left(z_{\alpha \beta}=\gamma\right)=\frac{1}{3} \cdot \frac{1}{4} .
$$

As a result, both assignments will satisfy a proportion of $\frac{1}{12}$ of the possible constraints.

Finally, suppose none of the variables of the clause receives the same value in $\tau$ as it does in $\sigma$. If $\alpha \neq \alpha^{\prime}, \beta \neq \beta^{\prime}$ and $\gamma \neq \gamma^{\prime}$, then

$$
\begin{aligned}
\operatorname{Pr}\left(z_{\alpha \beta}=\gamma \wedge z_{\alpha^{\prime} \beta^{\prime}}=\gamma^{\prime}\right)= & \operatorname{Pr}\left(z_{\alpha \beta}=\gamma \wedge z_{\alpha \beta^{\prime}} \neq \gamma^{\prime} \wedge z_{\alpha^{\prime} \beta^{\prime}}=\gamma^{\prime}\right) \\
= & \operatorname{Pr}\left(z_{\alpha^{\prime} \beta^{\prime}}=\gamma^{\prime} \mid z_{\alpha \beta}=\gamma \wedge z_{\alpha \beta^{\prime}} \neq \gamma^{\prime}\right) \\
& \times \operatorname{Pr}\left(z_{\alpha \beta^{\prime}} \neq \gamma^{\prime} \mid z_{\alpha \beta}=\gamma\right) \operatorname{Pr}\left(z_{\alpha \beta}=\gamma\right)
\end{aligned}
$$


Consider only the tuples $\left(\alpha, \beta, z_{\alpha \beta}\right),\left(\alpha, \beta^{\prime}, z_{\alpha \beta^{\prime}}\right)$, and $\left(\alpha^{\prime}, \beta^{\prime}, z_{\alpha^{\prime} \beta^{\prime}}\right)$ permitted by the constraint. There are 4 choices for $z_{\alpha \beta}$, and exactly $\frac{1}{4}$ of the constraints will have $z_{\alpha \beta}=\gamma$. For each constraint that contains the tuple $(\alpha, \beta, \gamma)$, there are exactly 3 choices for $z_{\alpha \beta^{\prime}}$, and 2 of these choices are not $\gamma^{\prime}$. By a straightforward symmetry argument, each is equally likely. As a result, $\frac{2}{3}$ of the constraints that contain the tuple $(\alpha, \beta, \gamma)$ will not contain the tuple $\left(\alpha, \beta^{\prime}, \gamma^{\prime}\right)$. Finally, for every choice of $z_{\alpha \beta}$ and $z_{\alpha \beta^{\prime}}$ there are 3 equally likely choices for $z_{\alpha^{\prime} \beta^{\prime}}$. The reason is that we fix the set of 3 -tuples that start with $\alpha$, and then the number of ways we can choose the set of tuples that starts with $\alpha^{\prime}$ is equal to the number of derangements on 4 elements, and by symmetry each derangement is equally likely.

As a result, the expression above yields:

$$
\operatorname{Pr}\left(z_{\alpha \beta}=\gamma \wedge z_{\alpha^{\prime} \beta^{\prime}}=\gamma^{\prime}\right)=\frac{1}{3} \cdot \frac{2}{3} \cdot \frac{1}{4} .
$$

So both assignments will simultaneously satisfy a proportion of $\frac{1}{18}$ of the possible constraints.

Using the same notation as [27, let $I_{k}=\left\{0, \frac{1}{k}, \frac{2}{k}, \ldots, \frac{k-1}{k}, 1\right\}$, and let $\alpha \in I_{n}$ be the proportion of variables having the same value in both assignments. To enumerate all pairs of assignments, we must count the number of choices for the $\alpha n$ variables and count the possible assignments to the variables. This gives $\sum_{\alpha \in I_{n}}\left(\begin{array}{c}n \\ \alpha n\end{array}\right) 4^{n} 3^{(1-\alpha) n}$ pairs of assignments.

To enumerate all satisfied instances for one pair of assignments, let $r \in I_{3 m}$ be the proportion of $3 \mathrm{~m}$ "places" in the second assignment that receive one of the $\alpha n$ variables. Note that if $\alpha=0$ then $r=0$, and if $\alpha=1$ then $r=1$. Otherwise, because each variable occurs in at least two places, we must have $r \geq \frac{2 \alpha}{3 c}$ and $1-r \geq \frac{2(1-\alpha)}{3 c}$.

Let $T_{k}$ be the number of clauses with $3-k$ of these $\alpha n$ variables. Recall that $T_{1}=0$. For each choice of $T_{0}, T_{2}, T_{3}$, we need to

(a) count the ways to choose the clauses for $T_{0}, T_{2}, T_{3}$ :

$$
\left(\begin{array}{c}
m \\
T_{0}
\end{array}\right)\left(\begin{array}{c}
m-T_{0} \\
T_{2}
\end{array}\right)
$$

(b) for each clause, count the number of ways we can choose a constraint for the clause given the number of variables in the clause that receive the same value in $\tau$ as in $\sigma$ :

$$
\left(\frac{q}{18}\right)^{T_{3}}\left(\frac{q}{12}\right)^{T_{2}}\left(\frac{q}{4}\right)^{T_{0}}
$$

(c) for each of the $T_{2}$ clauses that have exactly one of the $\alpha n$ variables, count the 3 positions for the $\alpha n$ variables:

$$
3^{T_{2}}
$$

(d) finally, distribute the variables amongst the "places". Recall that $S(i, j, 2)$ counts the number of ways to partition $i$ elements into $j$ sets such that each set 
has at least 2 elements. Also, we let $\Lambda_{3 m, n}\left(\alpha, r, T_{0}, T_{2}, T_{3}\right)$ denote the probability that this distribution of "places" is such that each clause contains three distinct variables. So the total number of choices for this step is:

$$
S(r 3 m, \alpha n, 2)(\alpha n) ! S((1-r) 3 m,(1-\alpha) n, 2)((1-\alpha) n) ! \Lambda_{3 m, n}\left(\alpha, r, T_{0}, T_{2}, T_{3}\right) .
$$

As a result, for a given $\alpha, r, T_{0}, T_{2}$, and $T_{3}$, the number of instances of $\Psi_{n, m}$ satisfied by a pair of assignments that fit the given parameters is

$$
\begin{aligned}
& \left(\begin{array}{c}
n \\
\alpha n
\end{array}\right) 4^{n} 3^{(1-\alpha) n}\left(\begin{array}{c}
m \\
T_{0}
\end{array}\right)\left(\begin{array}{c}
m-T_{0} \\
T_{2}
\end{array}\right)\left(\frac{q}{18}\right)^{T_{3}}\left(\frac{q}{12}\right)^{T_{2}}\left(\frac{q}{4}\right)^{T_{0}} 3^{T_{2}} \\
& \quad \times S(r 3 m, \alpha n, 2)(\alpha n) ! S((1-r) 3 m,(1-\alpha) n, 2)((1-\alpha) n) ! \Lambda_{3 m, n}\left(\alpha, r, T_{0}, T_{2}, T_{3}\right) .
\end{aligned}
$$

Let $t \in I_{m}=\left\{0, \frac{1}{m}, \frac{2}{m}, \ldots, \frac{m-1}{m}, 1\right\}$ be the proportion of $m$ clauses in which all 3 variables have the same assignment. Thus,

$$
\begin{aligned}
& T_{0}=t m \\
& T_{2}=3 r m-3 T_{0}=3 r m-3 t m \\
& T_{3}=m-T_{0}-T_{2}=m-3 r m+2 t m .
\end{aligned}
$$

Note that $T_{2} \geq 0$ implies $r \geq t$ and $T_{3} \geq 0$ implies $t \geq \frac{3 r-1}{2}$. Note also that if $\alpha=0$ then $r=0, T_{0}=T_{2}=0$ and $T_{3}=m$. Likewise, if $\alpha=1$ then $r=1$, $T_{0}=m$ and $T_{2}=T_{3}=0$.

Definition 14 (a) We say that $(\alpha, r, t) \in I_{n} \times I_{3 m} \times I_{m}$ is feasible if it satisfies $0 \leq \alpha \leq 1, \frac{2 \alpha}{3 c} \leq r \leq 1-\frac{2(1-\alpha)}{3 c}, \max \left(0, \frac{3 r-1}{2}\right) \leq t \leq r$ where if $\alpha=0$ then $r=t=0$, and if $\alpha=1$ then $r=t=1$.

(b) We say that $(\alpha, r, t)$ is extremal if it is feasible and either $\alpha=0, \alpha=$ $1, r=\frac{2 \alpha}{3 c}, r=1-\frac{2(1-\alpha)}{3 c}, t=\max \left(0, \frac{3 r-1}{2}\right)$ or $t=r$.

Setting $\Lambda_{3 m, n}^{\alpha, r, t}=\Lambda_{3 m, n}\left(\alpha, r, T_{0}, T_{2}, T_{3}\right)$, substituting and factoring out common terms yields that $\mathbf{E}\left(N^{2}\right)$ is the sum over all feasible $(\alpha, r, t)$ of:

$$
\begin{aligned}
F(\alpha, r, t)= & \frac{1}{S(3 m, n, 2) n ! \Lambda_{3 m, n}} \times\left(\begin{array}{c}
n \\
\alpha n
\end{array}\right) 4^{n} 3^{(1-\alpha) n} \\
& \times\left(\frac{m !}{(m-3 r m+2 t m) !(3 r m-3 t m) !(t m) !}\right) 3^{3 r m-3 t m} \\
& \times\left(\frac{1}{4}\right)^{m}\left(\frac{1}{3}\right)^{2 m+t m-3 r m} 2^{m-3 r m+2 t m} \\
& \times S(r 3 m, \alpha n, 2)(\alpha n) ! S((1-r) 3 m,(1-\alpha) n, 2)((1-\alpha) n) ! \Lambda_{3 m, n}^{\alpha, r, t} .
\end{aligned}
$$

We will treat the cases where $\alpha, r, t$ are close to the endpoints of their ranges separately. So we will take a constant $\zeta>0$ (to be specified in Section 6.4) and we define:

$$
\begin{aligned}
& I_{n}^{+}=I_{n} \cap[\zeta, 1-\zeta], \quad I_{3 m}^{+}=I_{3 m} \cap\left[\frac{2 \alpha+\zeta}{3 c}, 1-\frac{2(1-\alpha)+\zeta}{3 c}\right], \\
& I_{m}^{+}=I_{m} \cap\left[\max \left(0, \frac{3 r-1}{2}\right)+\zeta, r-\zeta\right] .
\end{aligned}
$$


In Section 6.4 we will prove:

Lemma 15 Let $c>\frac{2}{3}(1+2 \zeta)$. The sum of $F(\alpha, r, t)$ over all feasible $\alpha, r, t$ not satisfying $\alpha \in I_{n}^{+}, r \in I_{3 m}^{+}, t \in I_{m}^{+}$, is o $\left(4^{2 n-2 m}\right)$.

In Section 6.4 we choose a $\zeta$ small enough that $\frac{2}{3}(1+2 \zeta)<0.818469 \ldots$, the threshold for a non-empty 2 -core. Thus, we can assume $c>\frac{2}{3}(1+2 \zeta)$ and so Lemma 15 yields

$$
\mathbf{E}\left(N^{2}\right)=o\left(\mathbf{E}(N)^{2}\right)+\sum_{\alpha \in I_{n}^{+}} \sum_{r \in I_{3 m}^{+}} \sum_{t \in I_{m}^{+}} F(\alpha, r, t) .
$$

Notation: We use $f \sim g$ to mean that $f=g+o(g)$.

In the next few steps, we will use similar techniques as 27 to approximate $\mathbf{E}\left(N^{2}\right)$. First, we will apply approximations for the $S$ terms of $F$. Given Lemma 15, we can restrict the analysis to $c>\frac{2}{3}(1+2 \zeta)$ and points satisfying $\alpha \in I_{n}^{+}, r \in I_{3 m}^{+}, t \in I_{m}^{+}$. In this domain, each of the $S$ terms is of the form $S(i, j, 2)$ where $j>\zeta n>\frac{\zeta}{3 c} i$ and $j<\frac{i}{2}-\frac{\zeta}{2} n<\frac{i}{2}\left(1-\frac{\zeta}{2}\right)$. To approximate the $S$ terms, we use Lemma 24 which will be presented in Section 6.5. This lemma appears in [27] but with a typographical error. The lemma is also a specific case of the general results in 39. It gives the following approximation that holds uniformly as $i \rightarrow \infty$ for all $\delta_{1} i<j \leq \frac{i}{2}\left(1-\delta_{2}\right)$ for any positive constants $\delta_{1}$ and $\delta_{2}$ :

$$
S(i, j, 2) \sim \frac{1}{j !}\left(\frac{i}{z_{0} \mathrm{e}}\right)^{i}\left(\mathrm{e}^{z_{0}}-1-z_{0}\right)^{j} \Phi(i, j)
$$

where $z_{0}$ is the positive real solution of the equation

$$
\frac{j}{i} z_{0}=\frac{\mathrm{e}^{z_{0}}-1-z_{0}}{\mathrm{e}^{z_{0}}-1}
$$

and where

$$
\Phi(i, j)=\sqrt{\frac{i j}{z_{0} j(i-j)-i(i-2 j)}} .
$$

If we rewrite (33) as $\frac{i}{j}=\frac{z_{0}\left(\mathrm{e}^{z_{0}}-1\right)}{\mathrm{e}^{z_{0}}-1-z_{0}}$, note that the right hand side is a monotonically increasing function of $z_{0}$. Also, $\lim _{z_{0} \rightarrow 0} \frac{z_{0}\left(\mathrm{e}^{z_{0}}-1\right)}{\mathrm{e}^{z_{0}}-1-z_{0}}=2$ and $\lim _{z_{0} \rightarrow \infty} \frac{z_{0}\left(\mathrm{e}^{z_{0}}-1\right)}{\mathrm{e}^{z_{0}}-1-z_{0}}=$ $\infty$. Given these facts, the following observation is straightforward.

Observation 16 The function $z_{0}=z_{0}(i, j)$, defined by (3), is a continuous and differentiable function of $i$ and $j$ in the domain $i>2 j>0$. In addition, the limit of $z_{0}(i, j)$ as $(i, j)$ approaches $\left(2 j^{\prime}, j^{\prime}\right)$, for any $j^{\prime}>0$, exists and is 0 . 
For $\alpha \in I_{n}^{+}, r \in I_{3 m}^{+}, t \in I_{m}^{+}$, we have $j, i-2 j>\zeta n$ in each of the following three approximations, and so they hold uniformly as $n \rightarrow \infty$ for all such $\alpha, r, t$.

$$
\begin{aligned}
& S(3 m, n, 2) n ! \sim\left(\mathrm{e}^{x}-1-x\right)^{n} x^{-3 m} \mathrm{e}^{-3 m}(3 m)^{3 m} \Phi(3 m, n) \\
& S(r 3 m, \alpha n, 2)(\alpha n) ! \sim\left(\mathrm{e}^{z}-1-z\right)^{\alpha n} z^{-r 3 m} \mathrm{e}^{-r 3 m}(r 3 m)^{r 3 m} \Phi(r 3 m, \alpha n) \\
& S((1-r) 3 m,(1-\alpha) n, 2)((1-\alpha) n) ! \sim\left(\mathrm{e}^{y}-1-y\right)^{(1-\alpha) n} y^{-(1-r) 3 m} \\
& \mathrm{e}^{-(1-r) 3 m}((1-r) 3 m)^{(1-r) 3 m} \\
& \Phi((1-r) 3 m,(1-\alpha) n)
\end{aligned}
$$

for some $x, y, z>0$ such that

$$
\frac{\mathrm{e}^{x}-1-x}{\mathrm{e}^{x}-1}-\frac{x}{3 c}=\frac{\mathrm{e}^{y}-1-y}{\mathrm{e}^{y}-1}-\frac{y(1-\alpha)}{3 c(1-r)}=\frac{\mathrm{e}^{z}-1-z}{\mathrm{e}^{z}-1}-\frac{z \alpha}{3 c r}=0 .
$$

To complete our approximation of $F(\alpha, r, t)$, we apply Stirling's formula, $i ! \sim i^{i} \mathrm{e}^{-i} \sqrt{2 \pi i}$, to $m !,(m-3 \mathrm{rm}+2 \mathrm{tm}) !,(3 \mathrm{rm}-3 \mathrm{tm}) !,(t m) !$. For $t \in I_{m}^{+}$, we have $m-3 \mathrm{rm}+2 \mathrm{tm}, 3 \mathrm{rm}-3 \mathrm{tm}, \mathrm{tm} \geq \zeta \mathrm{m}$ and so this is an asymptotically tight approximation as $n \rightarrow \infty$. We then group the non-exponential and exponential terms and simplify. Lemma 15] along with the uniformity of our approximations for the $S(i, j, 2)$ terms and of Stirling's approximation, allow us to write $\mathbf{E}\left(N^{2}\right)$ in the form:

$$
\mathbf{E}\left(N^{2}\right) \sim o\left(E(N)^{2}\right)+\sum_{\alpha \in I_{n}^{+}} \sum_{r \in I_{3 m}^{+}} \sum_{t \in I_{m}^{+}} g(\alpha, r, t) \mathrm{e}^{n f(\alpha, r, t)}
$$

where

$$
\begin{aligned}
g(\alpha, r, t)= & \left(\Phi(3 m, n) \Lambda_{3 m, n}\right)^{-1} \Phi(r 3 m, \alpha n) \Phi((1-r) 3 m,(1-\alpha) n) \Lambda_{3 m, n}^{\alpha, r, t} \\
& \times \frac{1}{\sqrt{(2 \pi)^{3} n m^{2} \alpha(1-\alpha)(1-3 r+2 t)(3 r-3 t) t}} \\
f(\alpha, r, t)= & \frac{1}{n}[n \ln 4+(1-\alpha) n \ln 3-m \ln 4-m(2+t-3 r) \ln 3 \\
& +m(1-3 r+2 t) \ln 2+m(3 r-3 t) \ln 3-n \alpha \ln \alpha \\
& -n(1-\alpha) \ln (1-\alpha)-m(1-3 r+2 t) \ln (1-3 r+2 t) \\
& -m(3 r-3 t) \ln (3 r-3 t)-m t \ln t+\alpha n \ln \left(\mathrm{e}^{z}-1-z\right) \\
& -r 3 m \ln z-r 3 m+r 3 m \ln (r 3 m)+(1-\alpha) n \ln \left(\mathrm{e}^{y}-1-y\right) \\
& -(1-r) 3 m \ln y-(1-r) 3 m+(1-r) 3 m \ln ((1-r) 3 m) \\
& \left.-n \ln \left(\mathrm{e}^{x}-1-x\right)+3 m \ln x+3 m-3 m \ln (3 m)\right] .
\end{aligned}
$$

By considering each term of $f$ separately, and from Observation 16, we have:

Observation 17 For all $c>\frac{2}{3}, f(\alpha, r, t)$ is continuous and differentiable in the domain $0<\alpha<1, \frac{2 \alpha}{3 c}<r<1-\frac{2(1-\alpha)}{3 c}$, and $\max \left\{0, \frac{3 r-1}{2}\right\}<t<$ $r$. Furthermore, for every point $\left(\alpha_{1}, r_{1}, t_{1}\right)$ on the boundary of this domain $\lim _{(\alpha, r, t) \rightarrow\left(\alpha_{1}, r_{1}, t_{1}\right)} f(\alpha, r, t)$ exists. 
We fix $c$ and replace $m$ with $\mathrm{cn}$. Combining common terms, and dividing $f$ through by $n$ gives

$$
\begin{aligned}
g(\alpha, r, t)= & \left(\Phi(3 c n, n) \Lambda_{3 m, n}\right)^{-1} \Phi(r 3 c n, \alpha n) \Phi((1-r) 3 c n,(1-\alpha) n) \Lambda_{3 m, n}^{\alpha, r, t} \\
& \times \frac{1}{(2 \pi n)^{3 / 2} c \sqrt{\alpha(1-\alpha)(1-3 r+2 t)(3 r-3 t) t}} \\
f(\alpha, r, t)= & \ln 4-c \ln 4+(1-\alpha) \ln 3-c(2+t-3 r) \ln 3 \\
& +c(1-3 r+2 t) \ln 2-\alpha \ln \alpha-(1-\alpha) \ln (1-\alpha) \\
& -c(1-3 r+2 t) \ln (1-3 r+2 t)-c(3 r-3 t) \ln (r-t)-c t \ln t \\
& +r 3 c \ln r+(1-r) 3 c \ln (1-r)+\alpha \ln \left(\mathrm{e}^{z}-1-z\right)-r 3 c \ln z \\
& +(1-\alpha) \ln \left(\mathrm{e}^{y}-1-y\right)-(1-r) 3 c \ln y-\ln \left(\mathrm{e}^{x}-1-x\right) \\
& +3 c \ln x,
\end{aligned}
$$

and thus,

$$
\frac{E\left(N^{2}\right)}{E(N)^{2}} \sim \sum_{\alpha \in I_{n}^{+}} \sum_{r \in I_{3 c n}^{+}} \sum_{t \in I_{c n}^{+}} g(\alpha, r, t) e^{n(f(\alpha, r, t)-2(1-c) \ln 4)} .
$$

To analyze this sum, we will need a few technical lemmas about $f$ and $g$. We begin with the key - determining a global maximum for $f$ :

Lemma 18 For all $\frac{2}{3} \leq c<1$, the unique global maximum of $f(\alpha, r, t)$ in the domain $0<\alpha<1, \frac{2 \alpha}{3 c}<r<1-\frac{2(1-\alpha)}{3 c}$, and $\max \left\{0, \frac{3 r-1}{2}\right\}<t<r$ occurs at $f\left(\frac{1}{4}, \frac{1}{4}, \frac{1}{16}\right)=2(1-c) \ln 4$. Furthermore, for all .67 $\leq c \leq 1$, there exists a constant $b=b(c)$ such that at every extremal $(\alpha, r, t), f$ approaches a limit that is less than $2(1-c) \ln 4-b$.

The proof of this lemma is computer-aided. We provide an outline in Section [6 and then more details in the appendix.

The analysis of $g$ is not as delicate. The proofs of the following lemmas appear in Section [6. First, we show that $g$ is bounded:

Lemma 19 There exists a constant $\nu$, independent of $n$, such that for all $\alpha \in$ $I_{n}^{+}, r \in I_{3 m}^{+}$, and $t \in I_{m}^{+}$we have $g(\alpha, r, t) \leq \nu$.

Next we provide an approximation of $g(\alpha, r, t)$ when $\alpha, r, t$ are near the critical point from Lemma 18.

Lemma 20 If $\alpha=\frac{1}{4}+o(1), r=\frac{1}{4}+o(1), t=\frac{1}{16}+o(1)$ then

$$
g(\alpha, r, t)=\Phi(3 c n, n) \times(\pi n)^{-\frac{3}{2}} \frac{2^{6}}{9 c}(1+o(1)) .
$$


By these lemmas, it is straightforward (see Section 6) to show that the mass of the sum will occur when $\alpha, r, t$ are near $\frac{1}{4}, \frac{1}{4}, \frac{1}{16}$ and so we can replace $g(\alpha, r, t)$ by its approximation from Lemma 20, obtaining:

$$
\frac{E\left(N^{2}\right)}{E(N)^{2}} \sim \sum_{\alpha \in I_{n}^{+}} \sum_{r \in I_{3 c n}^{+}} \sum_{t \in I_{c n}^{+}} \Phi(3 c n, n) \times(\pi n)^{-\frac{3}{2}} \frac{2^{6}}{9 c} \mathrm{e}^{n(f(\alpha, r, t)-2(1-c) \ln 4)} .
$$

Next, we replace the summation with an integral. The summation is essentially a Riemann sum, and as $n$ tends to infinity, the error term from approximating the summation with an integral tends to 0.

$$
\begin{aligned}
\frac{\mathbf{E}\left(N^{2}\right)}{\mathbf{E}(N)^{2}} \sim & \Phi(3 c n, n) \times(\pi n)^{-\frac{3}{2}} \frac{2^{6}}{9 c} \sum_{\alpha \in I_{n}^{+}} \sum_{r \in I_{3 c n}^{+}} \sum_{t \in I_{c n}^{+}} \mathrm{e}^{n(f(\alpha, r, t)-2(1-c) \ln 4)} \\
\sim & \Phi(3 c n, n) \times(\pi n)^{-\frac{3}{2}} \frac{2^{6}}{9 c} \times n \times 3 c n \times c n \\
& \times \int_{\zeta}^{1-\zeta} \int_{\frac{2 \alpha+\zeta}{3 c}}^{1-\frac{2(1-\alpha)+\zeta}{3 c}} \int_{\max \left\{0, \frac{3 r-1}{2}\right\}+\zeta}^{r-\zeta} \mathrm{e}^{n(f(\alpha, r, t)-2(1-c) \ln 4)} d t d r d \alpha \\
< & \Phi(3 c n, n) \times n^{3 / 2} \frac{2^{6} c}{3 \pi^{3 / 2}} \\
& \times \int_{0}^{1} \int_{\frac{2 \alpha}{3 c}}^{1-\frac{2(1-\alpha)}{3 c}} \int_{\max \left\{0, \frac{3 r-1}{2}\right\}}^{r} \mathrm{e}^{n(f(\alpha, r, t)-2(1-c) \ln 4)} d t d r d \alpha .
\end{aligned}
$$

Continuing with the technique of 27, we approximate this sum by using the Laplace Method, as given in eg. 10] and 23. The Laplace Method for a triple integral can be stated as follows.

Lemma 21 ([23]) Let

$$
F(n)=\int_{a_{1}}^{b_{1}} \int_{a_{2}}^{b_{2}} \int_{a_{3}}^{b_{3}} \mathrm{e}^{n h\left(x_{1}, x_{2}, x_{3}\right)} d x_{1} d x_{2} d x_{3}
$$

where

(a) $h$ is continuous in $a_{i} \leq x_{i} \leq b_{i}$,

(b) $h\left(c_{1}, c_{2}, c_{3}\right)=0$ for some point $\left(c_{1}, c_{2}, c_{3}\right)$ with $a_{i}<c_{i}<b_{i}$ and $h\left(x_{1}, x_{2}, x_{3}\right)<0$ for all other points in the range,

(c) $h\left(x_{1}, x_{2}, x_{3}\right)=-\frac{1}{2} \sum_{i=1}^{3} \sum_{j=1}^{3} a_{i j} x_{i} x_{j}+\mathrm{o}\left(x_{1}^{2}+x_{2}^{2}+x_{3}^{2}\right)$ with $\left(x_{1}^{2}+x_{2}^{2}+x_{3}^{2} \rightarrow 0\right)$, and

(d) the quadratic form $\sum \sum a_{i j} x_{i} x_{j}$ is positive definite. 
Then,

$$
F(n) \sim(2 \pi)^{\frac{3}{2}} D^{-\frac{1}{2}} n^{-\frac{3}{2}}
$$

where $D$ is the determinant of the matrix $\left(a_{i j}\right)$.

Just as is done in 27, we apply Lemma 21 by letting $h\left(x_{1}, x_{2}, x_{3}\right)=$ $f(\alpha, r, t)-2(1-c) \ln 4$. By Observation 17, this satisfies point (a). Point (b) is satisfied by Lemma 18. Point (c) is satisfied if we approximate $h$ by the Taylor expansion about the point $\alpha=\frac{1}{4}, r=\frac{1}{4}, t=\frac{1}{16}$ and take the $a_{i j}$ 's from the second partial derivatives of $h$. Part (d) is satisfied by the following lemma which is proved in Section 6 .

Lemma 22 The quadratic form $\sum \sum a_{i j} x_{i} x_{j}$ is positive definite, and the determinant of the matrix $\left(a_{i j}\right)$ is $D=\frac{2^{15}}{9} c^{2}(\Phi(3 c n, n))^{2}$.

Now, we can apply the Laplace Method to obtain,

$$
\begin{aligned}
\frac{\mathbf{E}\left(N^{2}\right)}{\mathbf{E}(N)^{2}} & \sim \Phi(3 c n, n) \times n^{\frac{3}{2}} \frac{2^{6} c}{3 \pi^{3 / 2}} \times(2 \pi)^{\frac{3}{2}}\left(\frac{2^{15}}{9} c^{2}(\Phi(3 c n, n))^{2}\right)^{-\frac{1}{2}} n^{-\frac{3}{2}} \\
& \sim 1
\end{aligned}
$$

proving (11) and thus completing the proof of Lemma 13 .

\section{$6 \quad$ Further details}

Here we present most of the details that were postponed from the analysis in the previous section. We'll start with the straightforward derivation of (8). By Lemma 18, $f\left(\frac{1}{4}, \frac{1}{4}, \frac{1}{16}\right)$ is a global maximum. Therefore, there exists a function $\rho(n)=o(1)$ such that, defining $\Psi=\left\{(\alpha, r, t):\left|\alpha-\frac{1}{4}\right|,\left|r-\frac{1}{4}\right|,\left|t-\frac{1}{16}\right|>\rho(n)\right\}$, we have $f(\alpha, r, t)<f\left(\frac{1}{4}, \frac{1}{4}, \frac{1}{16}\right)-10 \log n / n$ for all $(\alpha, r, t) \in \Psi$. This and Lemma 19 imply that there exists a constant $\nu$ independent of $n$ such that

$$
\begin{aligned}
& \sum_{\alpha, r, t \in \Psi} g(\alpha, r, t) e^{n(f(\alpha, r, t)-2(1-c) \ln 4)} \\
& \quad<\sum_{\alpha, r, t \in \Psi} \nu \mathrm{e}^{n\left(f\left(\frac{1}{4}, \frac{1}{4}, \frac{1}{16}\right)-2(1-c) \ln 4-10 \log n / n\right)} \\
& \leq\left|I_{n}^{+}\right|\left|I_{3 c n}^{+}\right|\left|I_{c n}^{+}\right| \times \nu \times n^{-10} \mathrm{e}^{n\left(f\left(\frac{1}{4}, \frac{1}{4}, \frac{1}{16}\right)-2(1-c)\right)} \\
& =o\left(e^{n\left(f\left(\frac{1}{4}, \frac{1}{4}, \frac{1}{16}\right)-2(1-c)\right)}\right) .
\end{aligned}
$$

Thus, if for $\alpha, r, t \in \Psi$, we replace $g(\alpha, r, t)$ by any constant, specifically $g\left(\frac{1}{4}, \frac{1}{4}, \frac{1}{16}\right)$, then it will have a negligible effect on the sum. For $\alpha, r, t \notin \Psi$, Lemma 20 implies that we can also replace $g(\alpha, r, t)$ by $g\left(\frac{1}{4}, \frac{1}{4}, \frac{1}{16}\right) \sim \Phi(3 c n, n) \times(\pi n)^{-\frac{3}{2}} \frac{2^{6}}{9 c}$ and so obtain

$$
\frac{\mathbf{E}\left(N^{2}\right)}{\mathbf{E}(N)^{2}} \sim \sum_{\alpha \in I_{n}^{+}} \sum_{r \in I_{3 c n}^{+}} \sum_{t \in I_{c n}^{+}} \Phi(3 c n, n) \times(\pi n)^{-\frac{3}{2}} \frac{2^{6}}{9 c} \mathrm{e}^{n(f(\alpha, r, t)-2(1-c) \ln 4)},
$$

which is (8). 


\subsection{The global maximum}

We start with our proof that $f\left(\frac{1}{4}, \frac{1}{4}, \frac{1}{16}\right)$ is a global maximum. Recall our statement:

Lemma 18 For all $\frac{2}{3} \leq c<1$, the unique global maximum of $f(\alpha, r, t)$ in the domain $0<\alpha<1, \frac{2 \alpha}{3 c}<r<1-\frac{2(1-\alpha)}{3 c}$, and $\max \left\{0, \frac{3 r-1}{2}\right\}<t<r$ occurs at $f\left(\frac{1}{4}, \frac{1}{4}, \frac{1}{16}\right)=2(1-c) \ln 4$. Furthermore, for all $.67 \leq c \leq 1$, there exists a constant $b=b(c)$ such that at every extremal $(\alpha, r, t), f$ approaches a limit that is less than $2(1-c) \ln 4-b$.

Most of the computer-aided proof of this lemma is deferred to the appendix. We lay down some of the initial steps here.

If we differentiate $f$ with respect to $\alpha, r, t$, we get

$$
\begin{aligned}
& \frac{\partial f}{\partial \alpha}=D_{\alpha}\left[(1-\alpha) \ln 3-\alpha \ln \alpha-(1-\alpha) \ln (1-\alpha)+\alpha \ln \left(\mathrm{e}^{z}-1-z\right)\right. \\
&\left.-r 3 c \ln z+(1-\alpha) \ln \left(\mathrm{e}^{y}-1-y\right)-(1-r) 3 c \ln y\right] \\
&=- \ln 3-\ln \alpha+\ln (1-\alpha)+\ln \left(\mathrm{e}^{z}-1-z\right)-\ln \left(\mathrm{e}^{y}-1-y\right) \\
&+\left[\frac{\alpha\left(\mathrm{e}^{z}-1\right)}{\mathrm{e}^{z}-1-z}-\frac{3 r c}{z}\right] \frac{\partial z}{\partial \alpha}+\left[\frac{(1-\alpha)\left(\mathrm{e}^{y}-1\right)}{\mathrm{e}^{y}-1-y}-\frac{(1-r) 3 c}{y}\right] \frac{\partial y}{\partial \alpha} \\
&=- \ln 3-\ln \alpha+\ln (1-\alpha)+\ln \left(\mathrm{e}^{z}-1-z\right)-\ln \left(\mathrm{e}^{y}-1-y\right) \quad \text { by (4) }
\end{aligned}
$$

$$
\begin{aligned}
& \frac{\partial f}{\partial r}=D_{r} {[3 r c \ln 3-3 r c \ln 2-c(1-3 r+2 t) \ln (1-3 r+2 t)} \\
&-c(3 r-3 t) \ln (r-t)+r 3 c \ln r+(1-r) 3 c \ln (1-r)+\alpha \ln \left(\mathrm{e}^{z}-1-z\right) \\
&\left.-r 3 c \ln z+(1-\alpha) \ln \left(\mathrm{e}^{y}-1-y\right)-(1-r) 3 c \ln y\right] \\
&=3 c \ln 3-3 c \ln 2+3 c \ln (1-3 r+2 t)-3 c \ln (r-t)+3 c \ln r \\
&- 3 c \ln (1-r)-3 c \ln z+3 c \ln y+\left[\frac{\alpha\left(\mathrm{e}^{z}-1\right)}{\mathrm{e}^{z}-1-z}-\frac{r 3 c}{z}\right] \frac{\partial z}{\partial r} \\
&+ {\left[\frac{(1-\alpha)\left(\mathrm{e}^{y}-1\right)}{\mathrm{e}^{y}-1-y}-\frac{(1-r) 3 c}{y}\right] \frac{\partial y}{\partial r} } \\
&=3 c \ln 3-3 c \ln 2+3 c \ln (1-3 r+2 t)-3 c \ln (r-t)+3 c \ln r \quad \text { by (44) } \\
&- 3 c \ln (1-r)-3 c \ln z+3 c \ln y \\
& \frac{\partial f}{\partial t} \quad=\quad D_{t}[-c t \ln 3+c 2 t \ln 2-c(1-3 r+2 t) \ln (1-3 r+2 t) \\
&\quad-c(3 r-3 t) \ln (r-t)-c t \ln t] \\
& \quad-c \ln 3+2 c \ln 2-2 c \ln (1-3 r+2 t)+3 c \ln (r-t)-c \ln t .
\end{aligned}
$$


Setting $\frac{\partial f}{\partial \alpha}=\frac{\partial f}{\partial r}=\frac{\partial f}{\partial t}=0$ implies

$$
\begin{aligned}
\frac{1-\alpha}{\alpha} & =3 \frac{\mathrm{e}^{y}-1-y}{\mathrm{e}^{z}-1-z} \\
\frac{1-r}{r} & =\frac{3}{2} \cdot \frac{y}{z} \cdot \frac{(1-3 r+2 t)}{(r-t)} \\
\frac{(r-t)^{3}}{(1-3 r+2 t)^{2}} & =\frac{3 t}{4} .
\end{aligned}
$$

We will show that the maximum for $f$ is obtained by setting $x=y=z$. The intuition for this is that $x, y$, and $z$ correspond to the parameters for the truncated Poisson random variables used to model the degrees of all the variables in the 2-core, the variables in the 2-core that are not in the set of $\alpha n$ variables, and the variables in the 2-core that are the set of $\alpha n$ variables, respectively. Since $f$ is considering all possible sets of size $\alpha n$, it is reasonable to guess that the expected degrees for the variables in each set are equal when $f$ is maximized.

Plugging $x=y=z$ into (9), (10) and (11) gives $\alpha=\frac{1}{4}, r=\frac{1}{4}, t=\frac{1}{16}$ as a point where each of the partial derivatives are 0 . To confirm that this point is in the domain of $f$, we note that setting $\alpha=r$ in (44) yields $x=y=z$. Thus $\alpha=\frac{1}{4}, r=\frac{1}{4}, t=\frac{1}{16}$ is a stationary point of $f$ with

$$
f\left(\frac{1}{4}, \frac{1}{4}, \frac{1}{16}\right)=2(1-c) \ln 4 .
$$

Lemma 22, proved below, establishes that this is a local maximum. In the appendix, we will present a computer-aided proof that it is a global maximum. As part of the proof, the computer program gives a rigorous upper bound of $f^{+}(\alpha, r, t) \geq \lim _{\left(\alpha^{\prime}, r^{\prime}, t^{\prime}\right) \rightarrow(\alpha, r, t)} f\left(\alpha^{\prime}, r^{\prime}, t^{\prime}\right)$ for each triple $\alpha, r, t$ on the boundary, and the program proves that $2(1-c) \ln 4-f^{+}(\alpha, r, t) \geq b$ where $b>0$ is a constant independent of $\alpha, r$, and $t$, but it may be dependent on $c$. 


\subsection{Proof of Lemma 22}

Recall the statement:

Lemma 22 The quadratic form $\sum \sum a_{i j} x_{i} x_{j}$ is positive definite, and the determinant of the matrix $\left(a_{i j}\right)$ is $D=\frac{2^{15}}{9} c^{2}(\Phi(3 c n, n))^{2}$.

Proof The second partial derivatives of $f$ are:

$$
\begin{aligned}
f_{\alpha \alpha} & =-\frac{1}{\alpha}-\frac{1}{1-\alpha}+\frac{\mathrm{e}^{z}-1}{\mathrm{e}^{z}-1-z} \frac{\partial z}{\partial \alpha}-\frac{\mathrm{e}^{y}-1}{\mathrm{e}^{y}-1-y} \frac{\partial y}{\partial \alpha} \\
f_{\alpha r} & =\frac{\mathrm{e}^{z}-1}{\mathrm{e}^{z}-1-z} \frac{\partial z}{\partial r}-\frac{\mathrm{e}^{y}-1}{\mathrm{e}^{y}-1-y} \frac{\partial y}{\partial r} \\
f_{\alpha t} & =0 \\
f_{r \alpha} & =\frac{-3 c}{z} \frac{\partial z}{\partial \alpha}+\frac{3 c}{y} \frac{\partial y}{\partial \alpha} \\
f_{r r} & =-\frac{3 c}{r-t}+\frac{3 c}{r}+\frac{3 c}{1-r}-\frac{9 c}{1-3 r+2 t}-\frac{3 c}{z} \frac{\partial z}{\partial r}+\frac{3 c}{y} \frac{\partial y}{\partial r} \\
f_{r t} & =\frac{3 c}{r-t}+\frac{6 c}{1-3 r+2 t} \\
f_{t \alpha} & =0 \\
f_{t r} & =\frac{3 c}{r-t}+\frac{6 c}{1-3 r+2 t} \\
f_{t t} & =-\frac{3 c}{r-t}-\frac{c}{t}-\frac{4 c}{1-3 r+2 t}
\end{aligned}
$$

where, from (4),

$$
\begin{aligned}
\frac{\partial z}{\partial \alpha} & =\frac{-z\left(\mathrm{e}^{z}-1\right)^{2}}{\alpha\left(\mathrm{e}^{z}-1\right)^{2}+3 r c\left(\mathrm{e}^{z}\left(\mathrm{e}^{z}-1-z\right)-\left(\mathrm{e}^{z}-1\right)^{2}\right)} \\
\frac{\partial z}{\partial r} & =\frac{\alpha z\left(\mathrm{e}^{z}-1\right)^{2}}{r\left[\alpha\left(\mathrm{e}^{z}-1\right)^{2}+3 r c\left(\mathrm{e}^{z}\left(\mathrm{e}^{z}-1-z\right)-\left(\mathrm{e}^{z}-1\right)^{2}\right)\right]} \\
\frac{\partial y}{\partial \alpha} & =\frac{y\left(\mathrm{e}^{y}-1\right)^{2}}{(1-\alpha)\left(\mathrm{e}^{y}-1\right)^{2}+3(1-r) c\left(\mathrm{e}^{y}\left(\mathrm{e}^{y}-1-y\right)-\left(\mathrm{e}^{y}-1\right)^{2}\right)} \\
\frac{\partial y}{\partial r} & =\frac{-(1-\alpha) y\left(\mathrm{e}^{y}-1\right)^{2}}{(1-r)\left[(1-\alpha)\left(\mathrm{e}^{y}-1\right)^{2}+3(1-r) c\left(\mathrm{e}^{y}\left(\mathrm{e}^{y}-1-y\right)-\left(\mathrm{e}^{y}-1\right)^{2}\right)\right]} .
\end{aligned}
$$

Since $x=y=z$ at the maximum and setting $K=\frac{c\left(\mathrm{e}^{x}-1\right)^{2}}{\left(\mathrm{e}^{x}-1\right)^{2}+3 c\left(\mathrm{e}^{x}-x \mathrm{e}^{x}-1\right)}$, we 
have

$$
\begin{aligned}
f_{\alpha \alpha}\left(\frac{1}{4}, \frac{1}{4}, \frac{1}{16}\right) & =-\frac{16}{3}-16 K \\
f_{\alpha r}\left(\frac{1}{4}, \frac{1}{4}, \frac{1}{16}\right) & =16 K \\
f_{\alpha t}\left(\frac{1}{4}, \frac{1}{4}, \frac{1}{16}\right) & =0 \\
f_{r \alpha}\left(\frac{1}{4}, \frac{1}{4}, \frac{1}{16}\right) & =16 K \\
f_{r r}\left(\frac{1}{4}, \frac{1}{4}, \frac{1}{16}\right) & =-24 c-16 K \\
f_{r t}\left(\frac{1}{4}, \frac{1}{4}, \frac{1}{16}\right) & =32 c \\
f_{t \alpha}\left(\frac{1}{4}, \frac{1}{4}, \frac{1}{16}\right) & =0 \\
f_{t r}\left(\frac{1}{4}, \frac{1}{4}, \frac{1}{16}\right) & =32 c \\
f_{t t}\left(\frac{1}{4}, \frac{1}{4}, \frac{1}{16}\right) & =-\frac{128}{3} c .
\end{aligned}
$$

Thus,

$$
\left(a_{i j}\right)=\left[\begin{array}{ccc}
\frac{16}{3}+16 K & -16 K & 0 \\
-16 K & 24 c+16 K & -32 c \\
0 & -32 c & \frac{128}{3} c
\end{array}\right] .
$$

The quadratic form is positive definite if the following determinants are all 
positive (see, e.g., [8] p. 152).

$$
\begin{aligned}
\left|a_{11}\right| & =\frac{16}{3}+16 K \\
\left|\begin{array}{ll}
a_{11} & a_{12} \\
a_{21} & a_{22}
\end{array}\right| & =\left(\frac{16}{3}+16 K\right)(24 c+16 K)-(16 K)^{2} \\
& =16^{2}\left(\left(\frac{1}{3}+K\right)\left(\frac{3}{2} c+K\right)-K^{2}\right) \\
& =2^{8}\left(\frac{c}{2}+\frac{K}{3}+\frac{3 c K}{2}\right) \\
\left|\begin{array}{lll}
a_{11} & a_{12} & a_{13} \\
a_{21} & a_{22} & a_{23} \\
a_{31} & a_{32} & a_{33}
\end{array}\right| & =\left(\frac{16}{3}+16 K\right)\left|\begin{array}{cc}
a_{22} & a_{23} \\
a_{32} & a_{33}
\end{array}\right|-(-16 K)\left|\begin{array}{ll}
a_{21} & a_{23} \\
a_{31} & a_{33}
\end{array}\right| \\
& =16\left(\left(\frac{1}{3}+K\right)\left[2^{10} c^{2}+\frac{2^{11} K c}{3}-2^{10} c^{2}\right]\right. \\
& =2^{15}\left(\frac{K c}{9}+\frac{K^{2} c}{3}-\frac{K^{2} c}{3}\right) \\
& =\frac{2^{15}}{9} K c .
\end{aligned}
$$

From (44), $\mathrm{e}^{x}=1+\frac{3 c x}{3 c-x}$. Using this, we can simplify $K$.

$$
\begin{aligned}
K & =\frac{c\left(\mathrm{e}^{x}-1\right)^{2}}{\left(\mathrm{e}^{x}-1\right)^{2}+3 c\left(\mathrm{e}^{x}-1-x \mathrm{e}^{x}\right)} \\
& =\frac{c(3 c x)^{2}}{(3 c x)^{2}+3 c(3 c x)(3 c-x)-3 c x(3 c-x)^{2}-(3 c x)^{2}(3 c-x)} \\
& =\frac{3 c^{2}}{x(3 c-1)-3 c(3 c-2)} \\
& =c \times \frac{3 c n^{2}}{x n(3 c n-n)-3 c n(3 c n-2 n)} \\
& =c(\Phi(3 c n, n))^{2} .
\end{aligned}
$$

Thus, $K \geq 0$, which yields that the determinants above are all positive and so the quadratic form is positive definite. This establishes that the determinant $D$ of $\left(a_{i j}\right)$ is $\frac{2^{15}}{9} K c$, as required. 


\subsection{Approximating $g$}

First we prove our bound on $g$. Recall that

$$
\begin{aligned}
g(\alpha, r, t) & =\left(\Phi(3 c n, n) \Lambda_{3 m, n}\right)^{-1} \Phi(r 3 c n, \alpha n) \Phi((1-r) 3 c n,(1-\alpha) n) \Lambda_{3 m, n}^{\alpha, r, t} \\
& \times \frac{1}{(2 \pi n)^{3 / 2} c \sqrt{\alpha(1-\alpha)(1-3 r+2 t)(3 r-3 t) t}}
\end{aligned}
$$

where

$$
\Phi(i, j)=\sqrt{\frac{i j}{z_{0} j(i-j)-i(i-2 j)}},
$$

and where $\Lambda_{3 m, n}$ is the probability that if we distribute $3 m$ objects from $m$ triples into $n$ sets such that set receives at least 2 objects, then we do not have two objects from the same triple in the same set. $\Lambda_{3 m, n}^{\alpha, r, t}$ is a similar probability but with the distribution further restricted by $\alpha, r$, and $t$. Our main job is to approximate the $\Lambda$ terms:

Lemma 23 Let $\alpha=\frac{1}{4}+o(1), r=\frac{1}{4}+o(1)$, and $t=\frac{1}{16}+o(1)$, then

$$
\Lambda_{3 m, n}=\Lambda_{3 m, n}^{\alpha, r, t}+o(1) .
$$

Proof We recast the setting slightly, focusing only on what is relevant to this lemma: we consider a random partition of $3 m$ elements into $n$ buckets, where the elements come in $m$ triples, and the partition is uniform conditional on each bucket containing at least two elements. $\Lambda_{3 m, n}$ is the probability that no bucket contains two elements from one triple.

Now suppose that we split the buckets into two groups $A, B$ with $|A|=$ $\alpha n,|B|=(1-\alpha) n$, and fix

- the total number of elements that land in the buckets of $A$ is $3 \mathrm{rm}$;

- the number of triples, all of whose elements lie in $A$, is $t m$.

$\Lambda_{3 m, n}^{\alpha, r, t}$ is the probability, under this conditioning, that no bucket contains two elements from one triple.

To approximate $\Lambda_{3 m, n}$, we will first expose the number of elements in each bucket, and then we will expose which elements go into the buckets. Let $B_{i}$ denote the number of elements in bucket $i$, and let

$$
\Upsilon=\sum_{i=1}^{n} B_{i}\left(B_{i}-1\right)
$$

Having exposed the bucket sizes, we take a uniformly random partition of the elements into parts matching the bucket sizes. Let $Y$ denote the number of 
pairs of elements that lie in the same triple and in the same bucket. There are $3 m$ pairs that lie in the same triple, and so

$$
\begin{aligned}
\mathbf{E}(Y)=\mu_{Y} & =3 m \times \sum_{i \geq 1} B_{i}\left(B_{i}-1\right) \times \frac{1}{(3 m)(3 m-1)} \\
& \sim \frac{1}{3 m} \times \Upsilon \\
& =\frac{1}{3 m} \Upsilon .
\end{aligned}
$$

For each $j \geq 2$ a straightforward calculation shows that the expected number of $j$-tuples of pairs that are counted by $Y$ is $\mathbf{E}(Y)^{j}+o(1)$; the $o(1)$ term comes from the small probability that all three elements of a triple lie in the same bucket, and from the fact that the $\frac{1}{(3 m)(3 m-1)}$ term becomes $\frac{1}{(3 m)(3 m-1) \ldots(3 m-2 j+1)}$ rather than $\left(\frac{1}{(3 m)(3 m-1)}\right)^{j}$. Thus, the "method of moments" (see, eg. Section 6.1 of [44) implies that $Y$ is asymptotically distributed like a Poisson and so

$$
\Lambda_{3 m, n}=\operatorname{Pr}(Y=0)=\mathrm{e}^{-\mu_{Y}}+o(1)
$$

For $\Lambda_{3 m, n}^{\alpha, r, t}$, we take the same approach, first dealing with the buckets in $A$ and then with those in $B$. Let $B_{i}^{\prime}$ denote the the number of elements in bucket $i$ (and note that since this is a different experiment, we do not necessarily have $\left.B_{i}=B_{i}^{\prime}\right)$. Then let

$$
\Upsilon_{A}=\sum_{i \in A} B_{i}^{\prime}\left(B_{i}^{\prime}-1\right) ; \quad \Upsilon_{B}=\sum_{i \in B} B_{i}^{\prime}\left(B_{i}^{\prime}-1\right) .
$$

The number of triples with all three elements in $A$ is $t m$, and as discussed in Section 5 no triples have two elements in $A$. So letting $Y_{A}$ denote the number of pairs of elements that lie in the same triple and in the same bucket of $A$, we have:

$$
\begin{aligned}
\mathbf{E}\left(Y_{A}\right)=\mu_{A} & =3 t m \times \sum_{i \in A} B_{i}^{\prime}\left(B_{i}^{\prime}-1\right) \times \frac{1}{(3 r m)(3 r m-1)} \\
& \sim \frac{t}{3 r^{2} m} \times \Upsilon_{A} \\
& \sim \frac{1}{3 m} \Upsilon_{A} .
\end{aligned}
$$

From Section [5] the number of triples with all three elements in $B$ is $T_{3}=$ $m-3 \mathrm{rm}+2 \mathrm{tm}$ and the number with two elements in $B$ is $T_{2}=3 \mathrm{rm}-3 \mathrm{tm}$. 
So, defining $Y_{B}$ analogously to $Y_{A}$, we have:

$$
\begin{aligned}
\mathbf{E}\left(Y_{B}\right)=\mu_{B}= & (3(m-3 r m+2 t m)+(3 r m-3 t m)) \times \sum_{i \in B} B_{i}^{\prime}\left(B_{i}^{\prime}-1\right) \\
& \times \frac{1}{(3(1-r) m)(3(1-r) m-1)} \\
\sim & \frac{1-2 r+t}{3(1-r)^{2} m} \times \Upsilon_{B} \\
\sim & \frac{1}{3 m} \Upsilon_{B} .
\end{aligned}
$$

Again, the method of moments yields:

$$
\Lambda_{3 m, n}^{\alpha, r, t}=\operatorname{Pr}\left(Y_{A}=0\right) \operatorname{Pr}\left(Y_{B}=0\right)=\mathrm{e}^{-\mu_{A}-\mu_{B}}+o(1) .
$$

We will show below that a.a.s. $\Upsilon=\Upsilon_{A}+\Upsilon_{B}+o(1)$ and so $\mu_{Y}=\mu_{A}+\mu_{B}+o(1)$, thus yielding the lemma.

To study $\Upsilon, \Upsilon_{A}, \Upsilon_{B}$, we will apply Poissonization to allow us to treat $B_{i}, B_{i}^{\prime}$ as independent variables. (See Section 5.4 of [55] for a discussion of this technique.) Let $Z(\mu)$ denote a Poisson variable with mean $\mu$ and let $Z_{\geq_{2}}(\mu)$ denote the same variable but truncated as being at least two; i.e., $\operatorname{Pr}\left(Z_{\geq 2}(\mu)=i\right)$ is 0 for $i<2$ and is $\operatorname{Pr}(Z(\mu)=i) / \operatorname{Pr}(Z(\mu) \geq 2)$ otherwise. Let $b_{1}, \ldots, b_{n}$ be independent variables distributed like $Z_{\geq_{2}}(3 \mathrm{~m} / n)$ and let $b$ be the random vector $\left(b_{1}, \ldots, b_{n}\right)$ conditional on the event $E$ that $b_{1}+\ldots+b_{n}=3 m$. It is straightforward to show that this vector has the same distribution as the vector $\left(B_{1}, \ldots, B_{n}\right)$. Indeed, for any $x_{1}, \ldots, x_{n} \geq 2$ that sum to $3 m$, the probability that $\left(B_{1}, \ldots, B_{n}\right)=\left(x_{1}, \ldots, x_{n}\right)$ is

$$
\left(\begin{array}{c}
3 m \\
x_{1}, \ldots, x_{n}
\end{array}\right) \frac{1}{S(3 m, n, 2)}
$$

and the probability that $\left(b_{1}, \ldots, b_{n}\right)=\left(x_{1}, \ldots, x_{n}\right)$ is

$$
\begin{aligned}
\frac{1}{\operatorname{Pr}(E)} \prod_{i=1}^{n} \frac{\operatorname{Pr}\left(Z(3 m / n)=x_{i}\right)}{\operatorname{Pr}(Z(3 m / n) \geq 2)} & =\frac{1}{\operatorname{Pr}(E) \operatorname{Pr}(Z(3 m / n) \geq 2)^{n}} \prod_{i=1}^{n} \mathrm{e}^{-3 m / n} \frac{(3 m / n)_{i}^{x}}{x_{i} !} \\
& =\frac{\mathrm{e}^{-3 m}(3 m / n)^{3 m}}{\operatorname{Pr}(E) \operatorname{Pr}(Z(3 m / n) \geq 2)^{n}} \times \frac{1}{\prod_{i=1}^{n} x_{i} !} .
\end{aligned}
$$

These probabilities are both proportional to $1 / \prod_{i=1}^{n} x_{i}$ ! and hence are both equal.

Let $x_{1}, \ldots, x_{n}$ be a sequence of $n$ independent variables distributed like $Z_{\geq 2}(3 m / n)$. Note that $\operatorname{Pr}(E)=\Theta\left(n^{-1 / 2}\right)$. Therefore, if a property fails with probability $o\left(n^{-1 / 2}\right)$ for $x_{1}, \ldots, x_{n}$ then it holds a.a.s. for $B_{1}, \ldots, B_{n}$. (In fact, for monotone properties, one can show that if it holds a.a.s. for $x_{1}, \ldots, x_{n}$ then it holds a.a.s. for $B_{1}, \ldots, B_{n}$, but we won't need this here.) Let $\Upsilon^{*}=\sum_{i=1}^{n} x_{i}\left(x_{i}-1\right)$. It is straightforward to show, eg. by Azuma's Inequality, that $\Upsilon^{*}$ is highly concentrated around it's mean; eg. that $\operatorname{Pr}\left(\left|\Upsilon^{*}-\mathbf{E}\left(\Upsilon^{*}\right)\right| \geq n^{2 / 3}\right) \ll n^{-1 / 2}$. Therefore, a.a.s. $\left|\Upsilon-\mathbf{E}\left(\Upsilon^{*}\right)\right|<n^{2 / 3}$. 
The same analysis shows that a.a.s. $\Upsilon_{A}, \Upsilon_{B}$ are concentrated around $\mathbf{E}\left(\Upsilon_{A}^{*}\right)$, $\mathbf{E}\left(\Upsilon_{B}^{*}\right)$, where $\Upsilon_{A}^{*}, \Upsilon_{B}^{*}$ are defined in the same manner except that the means of the truncated Poisson are $3 r m / \alpha n, 3(1-r) m /(1-\alpha) n$ rather than $3 m / n$. Since $\alpha=r+o(1)$, we have $\mathbf{E}\left(\Upsilon_{A}^{*}\right)=\frac{|A|}{n} \mathbf{E}\left(\Upsilon^{*}\right)+o(1), \mathbf{E}\left(\Upsilon_{B}^{*}\right)=\frac{|B|}{n} \mathbf{E}\left(\Upsilon^{*}\right)+o(1)$ and so a.a.s. $\Upsilon=\Upsilon_{A}+\Upsilon_{B}+o(1)$ as required.

Remark: It seems fortuitous that we obtained $\mu_{A}+\mu_{B} \sim \mu_{Y}$ here. Had this not occurred, we still would have had $\Lambda_{3 m, n}=L \times \Lambda_{3 m, n}^{\alpha, r, t}+o(1)$, for some constant $L$ which would have yielded $\frac{\mathbf{E}\left(N^{2}\right)}{\mathbf{E}(N)^{2}} \sim L$. This, in turn would have showed that the probability of satisfiability is at least a constant. Then, a standard application of Friedgut's Theorem 32. would have shown that the probability of satisfiability is indeed $1-o(1)$. (See, eg. [1] for a similar argument.)

This now easily yields Lemma 20.

Lemma 20] If $\alpha=\frac{1}{4}+o(1), r=\frac{1}{4}+o(1), t=\frac{1}{16}+o(1)$ then

$$
g(\alpha, r, t)=\Phi(3 c n, n) \times(\pi n)^{-\frac{3}{2}} \frac{2^{6}}{9 c}+o(1) .
$$

Proof This follows immediately from Lemma 23 and a straightforward simplification.

We close this section with the proof of Lemma 19 .

Lemma 19 There exists a constant $\nu$, independent of $n$, such that for all $\alpha \in I_{n}^{+}, r \in I_{3 m}^{+}$, and $t \in I_{m}^{+}$we have $g(\alpha, r, t) \leq \nu$.

Proof We will consider the terms of $g(\alpha, r, t)$ separately. Recall that $I_{n}^{+}=[\zeta, 1-\zeta]$ for some constant $\zeta$. As a result, $\frac{1}{\sqrt{2 \pi n \alpha(1-\alpha)}}<1$ when $\alpha \in I_{n}^{+}$. Similarly, $\frac{1}{\sqrt{4 \pi^{2} m^{2}(1-3 r+2 t)(3 r-3 t) t}}<1$ when $t \in I_{m}^{+}$.

Next we consider the $\Phi$ terms of $g$. Let $\chi(i, j)=\sqrt{\frac{j(i-2 j)}{z_{0} j(i-j)-i(i-2 j)}}$ where $\frac{i}{j}=\frac{z_{0}\left(\mathrm{e}^{z_{0}}-1\right)}{\mathrm{e}^{z_{0}-1-z_{0}}}$, then $\Phi(i, j)=\chi(i, j) \times \frac{\sqrt{2 \pi i}}{\sqrt{2 \pi(i-2 j)}}$. We define $\chi\left(z_{0}\right)=\chi(i, j)$ 
where

$$
\begin{aligned}
& \chi(i, j)=\sqrt{\frac{j(i-2 j)}{z_{0} j(i-j)-i(i-2 j)}} \\
& =\sqrt{\frac{\frac{z_{0}\left(\mathrm{e}^{z_{0}}-1\right)}{\mathrm{e}^{z_{0}-1-z_{0}}-2}}{z_{0}\left(\frac{z_{0}\left(\mathrm{e}^{\left.z_{0}-1\right)}\right.}{\mathrm{e}^{z} 0-1-z_{0}}-1\right)-\frac{z_{0}\left(\mathrm{e}^{z_{0}}-1\right)}{\mathrm{e}^{z} 0-1-z_{0}}\left(\frac{z_{0}\left(\mathrm{e}^{\left.z_{0}-1\right)}\right.}{\left.\mathrm{e}^{z_{0}-1-z_{0}}-2\right)}\right.}} \\
& =\sqrt{\frac{z_{0}\left(\mathrm{e}^{z_{0}}-1\right)-2\left(\mathrm{e}^{z_{0}}-1\right)+2 z_{0}}{z_{0}\left(\mathrm{e}^{z_{0}}-1\right)+z_{0}^{2}\left(\frac{\mathrm{e}^{z_{0}-z_{0} \mathrm{e}^{z_{0}}-1}}{\mathrm{e}^{z_{0}}-1-z_{0}}\right)}} \\
& =\sqrt{\frac{z_{0}\left(\mathrm{e}^{z_{0}}-1\right)-2\left(\mathrm{e}^{z_{0}}-1-z_{0}\right)}{z_{0}\left(\mathrm{e}^{z_{0}}-1\right)-z_{0}^{2}\left(\frac{z_{0}\left(\mathrm{e}^{z_{0}}-1\right)}{\mathrm{e}^{z_{0}-1-z_{0}}}-1\right)}} \\
& =\chi\left(z_{0}\right) \text {. }
\end{aligned}
$$

As a result, we have

$$
\begin{gathered}
\Phi(r 3 c n, \alpha n)=\chi(z) \times \frac{\sqrt{2 \pi(3 r c n)}}{\sqrt{2 \pi(3 r c n-2 \alpha n)}} \\
\Phi((1-r) 3 c n,(1-\alpha) n)=\chi(y) \times \frac{\sqrt{2 \pi(3(1-r) c n)}}{\sqrt{2 \pi(3(1-r) c n-2(1-\alpha) n)}} \\
\Phi(3 c n, n)=\chi(x) \times \frac{\sqrt{2 \pi(3 c n)}}{\sqrt{2 \pi(3 c n-2 n)}} .
\end{gathered}
$$

It is straightforward to confirm that $\lim _{z_{0} \rightarrow 0} \chi\left(z_{0}\right)=1, \lim _{z_{0} \rightarrow \infty} \chi\left(z_{0}\right)=1$, $\chi\left(z_{0}\right)$ has a minimum at $z_{0} \approx 4.2$, and $0.84<\chi\left(z_{0}\right)<1$ for all positive $z_{0}$. In addition, each of the square root terms in (13) and (14) is $\Theta(\sqrt{n})$ because $r \in I_{3 m}^{+}$. Also, $\Phi(3 c n, n)^{-1}$ is $\mathrm{O}(1)$. (It is $\Theta(1)$ if $c$ is $\frac{2}{3}+\Theta(1)$, and it is $\Theta\left(n^{-1 / 2}\right)$ if $c$ is $\frac{2}{3}+O\left(n^{-1}\right)$. However, $r \in I_{3 m}^{+}$implies $c$ is $\frac{2}{3}+\Theta(1)$.) As a result, we have $\frac{\Phi(r 3 m, \alpha n) \Phi((1-r) 3 m,(1-\alpha n))}{\Phi(3 m, n)}=\mathrm{O}(1)$.

Finally, we consider the $\Lambda$ terms of $g$. Clearly, $\Lambda_{3 m, n}>0$, and in the proof of Lemma 23 it is implied that $\Lambda_{3 m, n}$ equals a constant independent of $n$ (because $\Upsilon$ is a.a.s. $\Theta(n), \mathbf{E}(Y)=\Theta(1)$, and thus $\operatorname{Pr}(Y=0)=\Theta(1))$. This proves that $\frac{\Lambda_{3 m, n}^{\alpha, r, t}}{\Lambda_{3 m, n}}=\mathrm{O}(1)$, and that completes the proof. 


\subsection{The border regions}

In this subsection, we handle the values of $\alpha, r, t$ that are close to the borders of the feasible region; i.e. we prove Lemma 15 .

Lemma 15 Let $c>\frac{2}{3}(1+2 \zeta)$. The sum of $F(\alpha, r, t)$ over all feasible $\alpha, r, t$ not satisfying $\alpha \in I_{n}^{+}, r \in I_{3 m}^{+}, t \in I_{m}^{+}$, is o( $\left.4^{2 n-2 m}\right)$.

Proof Recall that $m=c n$ for a fixed constant $c$, and so we can choose $\zeta$ to be arbitrarily small in terms of $c$. Note that if $c<\frac{2}{3}(1+2 \zeta)$ then the lemma does not hold because the global maximum at $\alpha=\frac{1}{4}, r=\frac{1}{4}, t=\frac{1}{16}$ has an $r$ coordinate smaller than $\frac{2 \alpha+\zeta}{3 c}$.

In this proof, we will approximate $F(\alpha, r, t)$ as in (5), except that we must be careful about certain terms. In particular, when we are too close to the border, some of the terms can be so small that our asymptotic approximations are not sufficiently close; so instead we will use the following absolute bounds.

The first are:

$$
\begin{aligned}
& i ! \geq\left(\frac{i}{\mathrm{e}}\right)^{i}, \\
& i !<\left(\frac{i}{\mathrm{e}}\right)^{i} \sqrt{2 \pi i+2},
\end{aligned}
$$

and

$$
\left(\begin{array}{l}
n \\
k
\end{array}\right) \leq\left(\frac{n \mathrm{e}}{k}\right)^{k}
$$

The next two bounds are on $S(i, j, 2) j !$. Recall that this is the number of ways to partition $i$ labeled elements into $j$ labeled buckets such that each bucket has at least 2 elements. The first bound is easily obtained by omitting the restriction that each bucket contain at least two elements:

$$
S(i, j, 2) j ! \leq j^{i} .
$$

The second is obtained by first choosing 2 elements for each bucket, and then distributing the remaining elements. Clearly, different choices can yield the same partition, and so this is an overcount:

$$
S(i, j, 2) j ! \leq\left(\begin{array}{c}
i \\
2 j
\end{array}\right) \frac{(2 j) !}{2^{j}} \times j^{i-2 j} .
$$

Now we turn to the border regions. We consider 8 cases, and note that some $(\alpha, r, t)$ will lie in more than one case. In the first two cases, we prove that the sum of the triples that satisfy those cases is o $\left(4^{2}(n-m)\right)$, and this proves the lemma for these cases.

Case 1: $\alpha=0$ or $\alpha=1$.

If $\alpha=0$, we must have $r=t=0$, and $F(0,0,0)=2^{(2-c) n} 3^{(1-2 c) n}$. If $\alpha=1$, we must have $r=t=1$, and $F(1,1,1)=4^{n-m}$. 
In the cases that follow, we will assume $0<\alpha<1$.

Case 2: $\alpha>(1-\zeta)$.

This case is easily dealt with, since the number of pairs of assignments which agree on exactly $\alpha n$ variables is $4^{n}\left(\begin{array}{c}n \\ n-\alpha n\end{array}\right) 3^{n-\alpha n}$. So $F(\alpha, r, t)$ is bounded by the number of such pairs, times the probability that the first one is satisfied, which, from bound (18), is at most:

$$
4^{n}\left(\frac{\mathrm{e} n}{\zeta n}\right)^{\zeta n} 3^{\zeta n} 4^{-m}=4^{n-m}\left(\frac{3 \mathrm{e}}{\zeta}\right)^{\zeta n}<o\left(4^{2(n-m)} / n^{3}\right),
$$

for $\zeta$ sufficiently small. So the sum of $F(\alpha, r, t)$ over the $O\left(n^{3}\right)$ triples $(\alpha, r, t)$ satisfying Case 2 is $o\left(4^{2(n-m)}\right)$.

For the remaining cases, we will approximate $F(\alpha, r, t)$ as in (5), except that we must be careful about certain terms. In particular, when we are too close to the border, some of the terms can be so small that our asymptotic approximations are not sufficiently close; so instead we will use the absolute bounds from above. In each case below, we will note how much this increases the bound over that obtained in (5).

Case 3: $\alpha<\zeta$.

Instead of using the approximation $\left(\begin{array}{c}n \\ \alpha n\end{array}\right) \sim \alpha^{-\alpha n}(1-\alpha)^{-(1-\alpha) n} \frac{1}{\sqrt{2 \pi n \alpha(1-\alpha)}}$, we use (18) to get the bound $\left(\begin{array}{c}n \\ \alpha n\end{array}\right)<\left(\frac{\mathrm{e}}{\alpha}\right)^{\alpha n}$. If we first consider the exponential terms, this replacement results in an increase over the bound in (5) by a factor of

$$
\begin{aligned}
\frac{\mathrm{e}^{\alpha n} \alpha^{-\alpha n}}{\alpha^{-\alpha n}(1-\alpha)^{-(1-\alpha) n}} & =\mathrm{e}^{\alpha n}(1-\alpha)^{(1-\alpha) n} \\
& <\mathrm{e}^{\gamma n}
\end{aligned}
$$

So the bound on the exponential terms in (7) is increased by at most a constant $\gamma>0$ which can be made arbitrarily small by taking $\zeta$ to be sufficiently small. The replacement also removes the term $(2 \pi n \alpha(1-\alpha))^{-1 / 2}$ from (6). In the proof of Lemma 19 this term is bounded by 1, so this change does not alter the bound of Lemma 19,

Next we replace the term $S(r 3 m, \alpha n, 2)(\alpha n)$ !. If $r$ is small enough that Case 4 below applies, we make the replacement described in that case. Otherwise, we replace the approximation $S(r 3 m, \alpha n, 2)(\alpha n) ! \sim\left(\mathrm{e}^{z}-1-z\right)^{\alpha n}\left(\frac{3 r m}{\mathrm{e} z}\right)^{3 r m} \Phi(r 3 m, \alpha n)$ with the bound obtained from (19): $S(r 3 m, \alpha n, 2)(\alpha n) !<(\alpha n)^{3 r m}$. We will consider the exponential terms first. Note that this replacement results in an 
increase over the bound in (5) by a factor of

$$
\begin{aligned}
& \frac{(\alpha n)^{3 r m}}{\left(\mathrm{e}^{z}-1-z\right)^{\alpha n}\left(\frac{3 r m}{\mathrm{e} z}\right)^{3 r m}} \\
& =\mathrm{e}^{3 r c n}\left(\frac{z \alpha}{3 r c}\right)^{3 r c n}\left(\mathrm{e}^{z}-1-z\right)^{-\alpha n} \\
& =\mathrm{e}^{3 r c n}\left(\frac{\mathrm{e}^{z}-1-z}{\mathrm{e}^{z}-1}\right)^{3 r c n}\left(\mathrm{e}^{z}-1-z\right)^{-\alpha n} \\
& =\frac{\mathrm{e}^{3 r c n}}{\left(\mathrm{e}^{z}-1\right)^{\alpha n}}\left(\frac{\mathrm{e}^{z}-1-z}{\mathrm{e}^{z}-1}\right)^{3 r c n-\alpha n} \\
& =\left(\frac{\mathrm{e}}{\left(\mathrm{e}^{z}-1\right)^{\frac{\mathrm{e}^{z}-1-z}{z\left(\mathrm{e}^{z}-1\right)}}}\right)^{3 r c n}\left(\frac{\mathrm{e}^{z}-1-z}{\mathrm{e}^{z}-1}\right)^{(3 r c-\alpha) n} \\
& <\mathrm{e}^{\gamma n} .
\end{aligned}
$$

So the bound on the exponential terms in (7) is increased by at most a constant $\gamma>0$ which can be made arbitrarily small by taking $\zeta$ to be sufficiently small. This follows from the fact that if $r$ is reasonably large so that Case 4 below does not apply, then as $\zeta$ and therefore $\alpha$ tends to $0, z$ tends to $\infty$, and $\lim _{z \rightarrow \infty}\left(\mathrm{e}^{z}-\right.$ $1)^{\frac{\mathrm{e}^{z}-1-z}{z\left(\mathrm{e}^{z}-1\right)}}=\mathrm{e}$.

This change also removes the term $\Phi(3 r m, \alpha n)$ from (6) , and from the proof of Lemma 19 this change increases the bound of Lemma 19 by at most a constant factor.

Case 4: $r<\frac{2 \alpha+\zeta}{3 c}$.

Rather than using the approximation

$$
S(r 3 m, \alpha n, 2)(\alpha n) ! \sim\left(\mathrm{e}^{z}-1-z\right)^{\alpha n}\left(\frac{3 r m}{\mathrm{e} z}\right)^{3 r m} \Phi(r 3 m, \alpha n),
$$

we will use the bound obtained from (20):

$$
\begin{aligned}
S(r 3 m, \alpha n, 2)(\alpha n) ! & <\left(\begin{array}{c}
r 3 m \\
2 \alpha n
\end{array}\right) \frac{(2 \alpha n) !}{2^{\alpha n}} \times(\alpha n)^{r 3 m-2 \alpha n} \\
& <\frac{(3 r m / \mathrm{e})^{3 r m}}{((3 r m-2 \alpha n) / \mathrm{e})^{3 r m-2 \alpha n}} 2^{-\alpha n}(\alpha n)^{3 r m-2 \alpha n} \sqrt{2 \pi 3 r m+2}
\end{aligned}
$$

with the last inequality following from bounds (17) and (16). Note that this replacement increases the bound in (5) by a factor of

$$
\begin{aligned}
& \frac{\frac{(3 r m / \mathrm{e})^{3 r m}}{((3 r m-2 \alpha n) / \mathrm{e})^{3 r m-2 \alpha n}} 2^{-\alpha n}(\alpha n)^{3 r m-2 \alpha n}}{\left(\mathrm{e}^{z}-1-z\right)^{\alpha n}\left(\frac{3 r m}{\mathrm{e} z}\right)^{3 r m}} \\
& =\left(\frac{\alpha n \mathrm{e}}{3 r m-2 \alpha n}\right)^{3 r m-2 \alpha n} \frac{z^{3 r m}}{\left(2\left(\mathrm{e}^{z}-1-z\right)\right)^{\alpha n}} \\
& <\mathrm{e}^{\gamma n} .
\end{aligned}
$$


So the bound on the exponential terms in (7) is increased by at most a constant $\gamma>0$ which can be made arbitrarily small by taking $\zeta$ to be sufficiently small. The last inequality holds because as $\zeta$ tends to $0,3 r c$ tends to $2 \alpha$ and $z$ tends to 0 , and $\lim _{z \rightarrow 0} \frac{z^{2}}{2\left(\mathrm{e}^{z}-1-z\right)}=1$.

This change also replaces the term $\Phi(3 r m, \alpha n)$ in (6) with $\sqrt{2 \pi 3 r m+2}$, and from the proof of Lemma 19 this change increases the bound of Lemma 19 by a factor of $\mathrm{O}(\sqrt{n})$.

Case 5: $r>1-\frac{2(1-\alpha)+\zeta}{3 c}$.

Rather than using the approximation

$$
\begin{gathered}
S((1-r) 3 m,(1-\alpha) n, 2)((1-\alpha) n) ! \sim\left(\mathrm{e}^{y}-1-y\right)^{(1-\alpha) n}\left(\frac{3(1-r) m}{\mathrm{e} y}\right)^{3(1-r) m} \\
\times \Phi((1-r) 3 m,(1-\alpha) n)
\end{gathered}
$$

we will use the bound obtained from (20):

$$
\begin{aligned}
S( & (1-r) 3 m, 1-\alpha n, 2)((1-\alpha) n) ! \\
< & \left(\begin{array}{c}
(1-r) 3 m \\
2(1-\alpha) n
\end{array}\right) \frac{(2(1-\alpha) n) !}{2^{(1-\alpha) n}} \times((1-\alpha) n)^{(1-r) 3 m-2(1-\alpha) n} \\
< & \frac{(3(1-r) m / \mathrm{e})^{3(1-r) m}}{((3(1-r) m-2(1-\alpha) n) / \mathrm{e})^{3(1-r) m-2(1-\alpha) n}} 2^{-(1-\alpha) n} \\
& \times((1-\alpha) n)^{3(1-r) m-2(1-\alpha) n} \sqrt{2 \pi 3(1-r) m+2}
\end{aligned}
$$

with the last inequality following from bounds (17) and (16). Note that, from the same argument as in Case 4, this replacement increases the bound in (5) by a factor of

$$
\begin{aligned}
& \frac{\frac{(3(1-r) m / \mathrm{e})^{3(1-r) m}}{((3(1-r) m-2(1-\alpha) n) / \mathrm{e})^{3(1-r) m-2(1-\alpha) n}} 2^{-(1-\alpha) n}((1-\alpha) n)^{3(1-r) m-2(1-\alpha) n}}{\left(\mathrm{e}^{y}-1-y\right)^{(1-\alpha) n}\left(\frac{3(1-r) m}{\mathrm{e} y}\right)^{3(1-r) m}} \\
& \quad=\left(\frac{(1-\alpha) n \mathrm{e}}{3(1-r) m-2(1-\alpha) n}\right)^{3(1-r) m-2(1-\alpha) n} \frac{y^{3(1-r) m}}{\left(2\left(\mathrm{e}^{y}-1-y\right)\right)^{(1-\alpha) n}} \\
& <\mathrm{e}^{\gamma n}
\end{aligned}
$$

So the bound on the exponential terms in (7) is increased by at most a constant $\gamma>0$ which can be made arbitrarily small by taking $\zeta$ to be sufficiently small.

This change also replaces the term $\Phi(3(1-r) m,(1-\alpha) n)$ in (6) with $\sqrt{2 \pi 3(1-r) m+2}$, and from the proof of Lemma 19 this change increases the bound of Lemma 19 by a factor of $\mathrm{O}(\sqrt{n})$.

Case 6: $t<\zeta$.

To obtain (5), we applied Stirling's formula and approximated $(\mathrm{tm})$ ! by $\left(\frac{t m}{\mathrm{e}}\right)^{t m} \sqrt{2 \pi t m}$. Instead, we will apply (16) and replace it with $\left(\frac{t m}{\mathrm{e}}\right)^{t m}$. This yields an upper bound on $F(\alpha, r, t)$ as $(t m)$ ! appears in the denominator. This 
replacement does not change the exponential terms of $F(\alpha, r, t)$, but it does remove the term $\sqrt{2 \pi t m}$ from (6), and from the proof of Lemma 19] this change increases the bound of Lemma 19 by at most a constant factor.

Case $7: t<\frac{3 r-1}{2}+\zeta$.

As in Case 6 , we will replace the $(m-3 r m+2 t m)$ ! term with $\left(\frac{m-3 r m+2 t m}{\mathrm{e}}\right)^{m-3 r m+2 t m}$. As in Case 6, this replacement does not change the exponential terms of $F(\alpha, r, t)$, but it does remove the term $\sqrt{2 \pi m(1-3 r+2 t)}$ from (6), and from the proof of Lemma 19 this change increases the bound of Lemma 19 by at most a constant factor.

Case 8: $t>r-\zeta$.

As in Cases 6 and 7 , we will replace the $(3 \mathrm{rm}-3 \mathrm{tm})$ ! term with $\left(\frac{3 \mathrm{rm}-3 \mathrm{tm}}{\mathrm{e}}\right)^{3 \mathrm{rm}-3 \mathrm{tm}}$. This replacement does not change the exponential terms of $F(\alpha, r, t)$, but it does remove the term $\sqrt{2 \pi m(3 r-3 t)}$ from (6), and from the proof of Lemma 19 this change increases the bound of Lemma 19 by at most a constant factor.

If $(\alpha, r, t)$ satisfy Cases 1 or 2 , then the lemma holds. If they satisfy any of Cases 3-8, then we obtain the upperbound

$$
F(\alpha, r, t) \leq(1+o(1)) g^{*}(\alpha, r, t) \mathrm{e}^{f^{*}(\alpha, r, t) n},
$$

where $g^{*}(\alpha, r, t)$ and $f^{*}(\alpha, r, t)$ are the result of applying the changes of Cases $3-8$ to (6) and (7).

Note that $g^{*}(\alpha, r, t) \leq \nu \times \mathrm{O}(n)<\nu \times \mathrm{e}^{\gamma n}$ where $\nu$ is the constant from Lemma 19, and note that $f^{*}(\alpha, r, t) \leq f(\alpha, r, t)+3 \gamma$ (to achieve this extreme bound, $(\alpha, r, t)$ would have to satisfy all Cases $3-5)$. Thus we have

$$
F(\alpha, r, t) \leq(1+o(1)) \nu \times \mathrm{e}^{\gamma n} \mathrm{e}^{(f(\alpha, r, t)+3 \gamma) n}=(1+o(1)) \mathrm{e}^{(f(\alpha, r, t)+4 \gamma) n} .
$$

Lemma 18 implies that if $\zeta$ is small enough to bound the borders of $I_{n}^{+} \times$ $I_{3 m}^{+} \times I_{m}^{+}$away from the unique global maximum point $\left(\frac{1}{4}, \frac{1}{4}, \frac{1}{16}\right)$ then there is some $x>0$, independent of $\zeta$, such that $f(\alpha, r, t)<2(1-c) \ln 4-x$ for any feasible $\alpha, r, t$ not satisfying $\alpha \in I_{n}^{+}, r \in I_{3 m}^{+}, t \in I_{m}^{+}$. We take $\zeta$ small enough to yield $\gamma=\frac{1}{8} x$, thus obtaining

$$
F(\alpha, r, t)<(1+o(1)) \mathrm{e}^{\left(2(1-c) \ln 4-\frac{1}{2} x\right) n},
$$

and we insure that $\zeta$ small enough so that $\frac{2}{3}(1+2 \zeta)<0.818469 \ldots$, the threshold for a non-empty 2-core (see Lemma 10), and thus we have $c>\frac{2}{3}(1+2 \zeta)$.

We need to be careful about one thing: if $(\alpha, r, t)$ is extremal - i.e. if $\alpha=0$, $\alpha=1, r=\frac{2 \alpha}{3 c}, r=1-\frac{2(1-\alpha)}{3 c}, t=0, t=\frac{3 r-1}{2}$ or $t=r$ - then $f(\alpha, r, t)$ and $g(\alpha, r, t)$ are undefined. In any such case, we obtain $F(\alpha, r, t) \leq(1+$ $o(1)) g^{*}(\alpha, r, t) \mathrm{e}^{f^{*}(\alpha, r, t) n}$ where $g^{*}(\alpha, r, t)<\lim _{\left(\alpha^{\prime}, r^{\prime}, t^{\prime}\right) \rightarrow(\alpha, r, t)} g\left(\alpha^{\prime}, r^{\prime}, t^{\prime}\right) \times \mathrm{e}^{\gamma n}$ and $f^{*}(\alpha, r, t) \leq \lim _{\left(\alpha^{\prime}, r^{\prime}, t^{\prime}\right) \rightarrow(\alpha, r, t)} f\left(\alpha^{\prime}, r^{\prime}, t^{\prime}\right)+3 \gamma$. So again, Lemmas 18 and 19 imply that

$$
F(\alpha, r, t)<(1+o(1)) \mathrm{e}^{\left(2(1-c) \ln 4-\frac{1}{2} x\right) n}<4^{2 n-2 m-\frac{1}{4} x n} .
$$


The asymptotics in this bound come from our approximations of factorial terms and of $S(i, j, 2)$ terms. In all cases, we either use approximations that hold uniformly over the given domain or we replace the approximations by bounds that hold over the domain, and so the asymptotic approximations and upper bounds hold uniformly. Thus we can sum them over the $O\left(n^{3}\right)$ triples and obtain the lemma.

\subsection{An Approximation for Generalized Stirling Numbers of the Second Kind}

Lemma 24 Let

$$
\Gamma(i, j)=\frac{1}{j !}\left(\frac{i}{z_{0} \mathrm{e}}\right)^{i}\left(\mathrm{e}^{z_{0}}-1-z_{0}\right)^{j} \sqrt{\frac{i j}{z_{0} j(i-j)-i(i-2 j)}}
$$

where $z_{0}$ is the positive real solution of the equation

$$
\frac{j}{i} z_{0}=\frac{\mathrm{e}^{z_{0}}-1-z_{0}}{\mathrm{e}^{z_{0}}-1} .
$$

Then

$$
S(i, j, 2) \sim \Gamma(i, j),
$$

and the approximation holds uniformly as $i \rightarrow \infty$ for all $\delta_{1} i<j<\frac{i}{2}\left(1-\delta_{2}\right)$ where $\delta_{1}$ and $\delta_{2}$ are any positive constants.

Proof Hennecart, in [39, extends the approximation by Temme [71] for Stirling numbers of the second kind, $S(i, j, 1)$ in the notation of this paper, to give the following approximation for the generalized Stirling number of the second kind $S(i, j, r)$.

$$
S(i, j, r) \sim \frac{i !}{j !(i-j r) !}\left(\frac{i-j r}{\mathrm{e}}\right)^{i-j r} \frac{B^{j}\left(z_{0}, r\right)}{z_{0}^{i+1}} \sqrt{\frac{j t_{0}}{\phi^{\prime \prime}\left(z_{0}\right)}}
$$

where $B(z, r)=e^{z}-\sum_{l=0}^{r-1} \frac{z^{l}}{l !}, \phi(z)=-i \ln z+j \ln B(z, r), t_{0}=\frac{i-j r}{j}$, and $z_{0}$ is the positive real solution of the equation $z_{0} \frac{B^{\prime}\left(z_{0}, r\right)}{B\left(z_{0}, r\right)}=\frac{i}{j}$.

If we let $r=2$, then $B(z, 2)=\mathrm{e}^{z}-1-z$, and

$$
\phi^{\prime \prime}(z)=\frac{i}{z^{2}}+j \frac{\mathrm{e}^{z}\left(\mathrm{e}^{z}-1-z\right)-\left(\mathrm{e}^{z}-1\right)^{2}}{\left(\mathrm{e}^{z}-1-z\right)^{2}} .
$$

Since, $z_{0} \frac{\mathrm{e}^{z_{0}}-1}{\mathrm{e}^{z_{0}}-1-z_{0}}=\frac{i}{j}$, we have

$$
\begin{aligned}
\mathrm{e}^{z_{0}} & =\frac{i+i z_{0}-j z_{0}}{i-j z_{0}} \\
\mathrm{e}^{z_{0}}-1 & =\frac{i z_{0}}{i-j z_{0}} \\
\mathrm{e}^{z_{0}}-1-z_{0} & =\frac{j z_{0}^{2}}{i-j z_{0}},
\end{aligned}
$$


and we can simplify $\phi^{\prime \prime}\left(z_{0}\right)$.

$$
\begin{aligned}
\phi^{\prime \prime}\left(z_{0}\right) & =\frac{i}{z_{0}^{2}}+j \frac{\left(i+i z_{0}-j z_{0}\right) j z_{0}^{2}-\left(i z_{0}\right)^{2}}{\left(j z_{0}^{2}\right)^{2}} \\
& =\frac{1}{j z_{0}^{2}}\left(z_{0} j(i-j)-i(i-2 j)\right) .
\end{aligned}
$$

This yields

$$
\begin{gathered}
S(i, j, 2) \sim \frac{i !}{j !(i-2 j) !}\left(\frac{i-2 j}{\mathrm{e}}\right)^{i-2 j} \frac{\left(\mathrm{e}^{z_{0}}-1-z_{0}\right)^{j}}{z_{0}^{i+1}} \\
\times \sqrt{\frac{j z_{0}^{2}(i-2 j)}{z_{0} j(i-j)-i(i-2 j)}} .
\end{gathered}
$$

Unfortunately, 39] states but does not prove that this approximation is uniform for the desired parameter values. As noted above, the [39] approximation for $S(i, j, r)$ was based on the [71] approximation for $S(i, j, 1)$, and the uniformity of the latter approximation was proven in [14. The 14 proof takes the error bounds for integer $i$ and $j$ of a slightly different approximation of $S(i, j, 1)$ by Moser and Wyman 62 and proves that these bounds also apply to the approximation of [71. In addition, [14] extend the proof of 62 to prove that the [71 approximation is also uniform for real and complex parameters. For the results of this paper, we require (21) to be uniform for integer $i$ and $j$ as $i \rightarrow \infty$ and for all $\delta_{1} i<j \leq \frac{i}{2}\left(1-\delta_{2}\right)$ where $\delta_{1}$ and $d_{2}$ are any positive constants. Sections 3 and 4 of [62] prove the error bounds for their approximation of $S(i, j, 1)$ for integer parameters with $0<j<i$ and $\lim _{i \rightarrow \infty}(i-j)=\infty$. In [17, we provide a modification of these error bounds to $S(i, j, 2)$, and this proves the desired uniformity of (21).

Applying Stirling's approximation to the $i$ ! and $(i-2 j)$ ! terms in (21), we obtain

$$
\begin{aligned}
S(i, j, 2) \sim \frac{1}{j !}\left[\left(\frac{i}{\mathrm{e}}\right)^{i}\left(\frac{\mathrm{e}}{i-2 j}\right)^{i-2 j} \frac{\sqrt{2 \pi i}}{\sqrt{2 \pi(i-2 j)}}\right]\left(\frac{i-2 j}{\mathrm{e}}\right)^{i-2 j} \\
\quad \times \frac{\left(\mathrm{e}^{z_{0}}-1-z_{0}\right)^{j}}{z_{0}^{i}} \sqrt{\frac{j(i-2 j)}{z_{0} j(i-j)-i(i-2 j)}} \\
=\frac{1}{j !}\left(\frac{i}{z_{0} \mathrm{e}}\right)^{i}\left(\mathrm{e}^{z_{0}}-1-z_{0}\right)^{j} \sqrt{\frac{i j}{z_{0} j(i-j)-i(i-2 j)}} \\
=\Gamma(i, j) .
\end{aligned}
$$

The error terms from applying Stirling's approximation to $i$ ! and $(i-2 j)$ ! are $\mathrm{O}\left(i^{-1}\right)$ and $\mathrm{O}\left((i-2 j)^{-1}\right)$, respectively, and these approximations are asymptotically tight as $i \rightarrow \infty$ with $i-2 j>\delta_{2} i$ for $\delta_{2}$ a positive constant. Therefore, we can conclude that the approximation of Lemma 24 is uniform for all $\delta_{1} i<j<\frac{i}{2}\left(1-\delta_{2}\right)$ as $i \rightarrow \infty$ with $\delta_{1}$ and $\delta_{2}$ any positive constants. 


\section{Conclusion}

In this paper, we present $(3,4)$-UE-CSP, the first known constraint satisfaction problem that has a random model with an exact satisfiability threshold as well as all of the following properties of random $k$-SAT, $k \geq 3$ : The problem is NPcomplete; the problem has constant size clauses and domain; the satisfiability threshold for the random model occurs when there are a linear number of clauses; a random instance a.a.s. has exponential resolution complexity; and there is no known polynomial time algorithm that, w.u.p.p., will find a solution to an instance drawn from close to the satisfiability threshold. In forming this CSP, we define UE-CSP as a general model of universal uniquely extendible CSPs that naturally generalize XOR-SAT.

Interestingly, the satisfiability threshold for random $(3,4)$-UE-CSP is exactly the same as for random 3-XOR-SAT. As noted above, 3-XOR-SAT is the same problem as $(3,2)$-UE-CSP. This observation leads to the question as to whether the location of the satisfiability threshold for random $(k, d)$-UE-CSP depends on the domain size $d$. We conjecture that it does not; i.e. that for every $k, d \geq 2$, the satisfiability threshold for random $(k, d)$-CSP is the same as for $k$-XORSAT, a threshold that was determined in 27]. [19] presents a proof for this conjecture, subject to a hypothesis that is analogous to Lemma 18 I.e., for any $k, d$, the conjecture holds so long as a natural local maximum of a particular function is the unique global maximum. With sufficient labor, this hypothesis could probably be proven for specific values of $k, d$ using computer-aided interval arithmetic analysis along the lines of that in our appendix. But proving that it holds for all $k, d$ would likely require a different approach.

\section{Acknowledgment}

We would like to thank two anonymous referees for their helpful suggestions.

\section{References}

[1] D. Achlioptas. A survey of lower bounds for random 3-SAT via differential equations. Theoretical Computer Science, 256(1-2):159-185, 2001.

[2] D. Achlioptas, P. Beame, and M. Molloy. A sharp threshold in proof complexity yields lower bounds for satisfiability search. Journal of Computer and System Sciences, 68:238-268, 2004.

[3] D. Achlioptas, A. Chtcherba, G. Istrate, and C. Moore. The phase transition in 1-in- $k$ SAT and NAE 3-SAT. In Proceedings of the Twelfth Annual ACM-SIAM Symposium on Discrete Algorithms, pages 721-722, 2001.

[4] D. Achlioptas, M. S. O. Molloy, L. M. Kirousis, Y. C. Stamatious, E. Kranakis, and D. Krizanc. Random constraint satisfaction: A more accurate picture. Constraints, 6(4):329-344, 2001. 
[5] D. Achlioptas and C. Moore. Random $k$-SAT: Two moments suffice to cross a sharp threshold. SIAM Journal of Computing, 36(3):740-762, 2006.

[6] D. Achlioptas and Y. Peres. The threshold for random $k$-SAT is $2^{k} \log 2-$ $\mathrm{O}(k)$. Journal of the American Mathematical Society, 17(4):947-973, 2004.

[7] F. Altarelli, R. Monasson, and F. Zamponi. Relationship between clustering and algorithmic phase transitions in the random k-XORSAT model and its NP-complete extensions. In Proceedings of the International Workshop on Statistical-Mechanical Informatics, 2007. arXiv:cs.CC:0709.0367v2 [cs.CC].

[8] T. M. Apostol. Mathematical Analysis: A Modern Approach to Advanced Calculus. Addison-Wesley, 1957 and 1974.

[9] E. Ben-Sasson and A. Wigderson. Short proofs are narrow - resolution made simple. Journal of the ACM, 48(2):149-169, 2001.

[10] C. Bender and S. Orsag. Advanced Mathematical Methods for Scientists and Engineers. McGraw-Hill, 1978.

[11] D. Le Berre and L. Simon. Fifty-five solvers in vancouver: The SAT 2004 competition. In Proceedings of the Seventh International Conference on Theory and Applications of Satisfiability Testing, pages 231-344, 2004.

[12] B. Bollobás. Graph Theory: An Introductory Course. Springer-Verlag, 1979.

[13] J. Cain and N. Wormald. Encores on cores. The Electronic Journal of Combinatorics, 13(1), 2006. R81.

[14] R. Chelluri, L. B. Richmond, and N. M. Temme. Asymptotic estimates for generalized stirling numbers. International Mathematical Journal of Analysis and its Applications, 20:1-13, 2000.

[15] V. Chvátal and B. Reed. Mick gets some (the odds are on his side). In Proceedings of the 33rd Annual IEEE Symposium on Foundations of Computer Science, pages 620-627. IEEE, 1992.

[16] V. Chvátal and E. Szemerédi. Many hard examples for resolution. Journal of the ACM, 35(4):759-768, 1988.

[17] H. Connamacher. On the uniformity of the approximation for generalized stirling numbers of the second kind. In preparation.

[18] H. Connamacher and M. Molloy. The exact satisfiability threshold for a potentially intractable random constraint satisfaction problem. In Proceedings of the 45th Annual IEEE Symposium on Foundations of Computer Science, pages 590-599, 2004.

[19] H. S. Connamacher. Threshold Phenomena in Random Constraint Satisfaction Problems. PhD thesis, University of Toronto, 2008. 
[20] J. M. Crawford and L. D. Auton. Experimental results on the crossover point in random 3-SAT. Artificial Intelligence, 81(1-2):31-57, 1996.

[21] N. Creignou and H. Daudé. Generalized satisfiability problems: Minimal elements and phase transitions. Theoretical Computer Science, 302(1-3):417430, June 2003.

[22] R. Darling and J. R. Norris. Cores and cycles in random hypergraphs. In preparation.

[23] N. G. de Bruijn. Asymptotic Methods in Analysis. North-Holland, 1958 and 1970. Dover reprint, 1981.

[24] W. Fernandez de la Vega. On random 2-SAT. Manuscript, 1992.

[25] J. Díaz, L. Kirousis, D. Mitsche, and X. Pérez-Giménez. A new upper bound for 3-SAT. In IARCS Annual Conference on Foundations of Software Technology and Theoretical Computer Science (FSTTCS 2008), volume 2 of Leibniz International Proceedings in Informatics, pages 163-174, 2008.

[26] M. Dietzfelbinger, A. Goerdt, M. Mitzenmacher, A. Montanari, R. Pagh, and M'Rink. Tight thresholds for cuckoo hashing via XORSAT. arXiv:0912.0287v3 [cs.DS], 2010.

[27] O. Dubois and J. Mandler. The 3-XORSAT threshold. In Proceedings of the 43rd Annual IEEE Symposium on Foundations of Computer Science, pages 769-778, 2002.

[28] O. Dubois and J. Mandler. The 3-xorsat threshold. C.R. Acad. Sci. Paris, Ser I, 335(11):963-966, 2002.

[29] M. Dyer, A. Frieze, and M. Molloy. A probabilistic analysis of randomly generated binary constraint satisfaction problems. Theoretical Computer Science, 290(3):1815-1828, 2003.

[30] A. D. Flaxman. A sharp threshold for a random constraint satisfaction problem. Discrete Mathematics, 285(1-3):301-305, 2003.

[31] J. Franco and M. Paull. Probabilistic analysis of the davis putnam procedure for solving the satisfiability problem. Discrete Applied Mathematics, $5(1): 77-87,1983$.

[32] E. Friedgut. Sharp thresholds of graph properties, and the $k$-SAT problem. Journal of the American Mathematical Society, 12(4):1017-1054, 1999.

[33] A. Frieze and N. C. Wormald. Random $k$-SAT: A tight threshold for moderately growing $k$. Combinatorica, 25:297-305, 2005.

[34] A. M. Frieze and M. Molloy. The satisfiability threshold for randomly generated binary constraint satisfaction problems. Rand. Struc. $\&$ Alg., 28:323-339, 2006. 
[35] I. P. Gent, E. Macintyre, P. Prosser, B. M. Smith, and T. Walsh. Random constraint satisfaction: Flaws and structure. Constraints, 6(4):345-372, 2001.

[36] A. Goerdt. A threshold for unsatisfiability. Journal of Computer and System Sciences, 53(3):469-486, 1996.

[37] M. T. Hajiaghayi and G. B. Sorkin. The satisfiability threshold of random 3-SAT is at least 3.52. arXiv:math/0310193v2 [math.CO], 2003.

[38] E. Hansen and G. W. Walster. Global Optimization Using Interval Analysis. Pure and Applied Mathematics. Marcel Dekker, second edition, 2004.

[39] F. Hennecart. Stirling distributions and stirling numbers of the second kind. computational problems and statistics. Kybernetika, 30(3):279-288, 1994 .

[40] M. J. H. Heule and H. van Maaren. March_dl: Adding adaptive heuristics and a new branching strategy. Journal on Satisfiability, Boolean Modeling, and Computation, 2:47-59, 2006.

[41] M. J. H. Heule and H. van Maaren. Effective incorporation of double lookahead procedures. In Theory and Applications of Satisfiability Testing SAT 200\%, Lecture Notes in Computer Science, pages 258-271. Springer, 2007.

[42] M. J. H. Heule and H. van Maaren. Whose side are you on? finding solutions in a biased search-tree. Journal on Satisfiablity, Boolean Modeling and Computation, 4:117-148, 2008.

[43] A. E. J. Hyvärinen, T. Junttila, and I. Niemelä. Incorporating learning in grid-based randomized SAT solving. In Artificial Intelligence: Methodology, Systems, and Applications, Lecture Notes in Computer Science, pages 247261. Springer, 2008.

[44] S. Janson, T. Łuczak, and A. Ruciński. Random Graphs. Wiley-Interscience Series in Discrete Mathematics and Optimization. John Wiley \& Sons, 2000.

[45] A. C. Kaporis, L. M. Kirousis, and E. G. Lalas. Selecting complementary pairs of literals. In Electronic Notes in Discrete Mathematics, volume 16. Elsevier, 2003.

[46] J. H. Kim. The poisson cloning model for random graphs. Located at URI research.microsoft.com/theory/jehkim/papers/PCMRG.pdf, 2006.

[47] S. Kirkpatrick and B. Selman. Critical behavior in the satisfiability of random boolean expressions. Science, 264(5163):1297-1232, 1994.

[48] Olaf Knüppel. PROFIL/BIAS V 2.0. Technical report, Technische Universität Hamburg - Harburg, 1999. 
[49] M. D. T. Lewis, T. Schubert, and B. W. Becker. Speedup techniques utilized in modern SAT solvers: An analysis in the MIRA environment. In Proceedings of the Eighth International Conference on Theory and Applications of Satisfiability Testing, pages 437-443, 2005.

[50] T. Euczak. On the equivalence of two basic models of random graphs. In Michal Karonski, Jerzy Jaworski, and Andrzej Rucinski, editors, Proceedings of Random Graphs '87, pages 151-158. John Wiley \& Sons, 1990.

[51] B. S. Majewski, N. C. Wormald, G. Havas, and Z. J. Czech. A family of perfect hashing methods. The Computer Journal, 39(6):547-554, 1996.

[52] M. Mézard, F. Ricci-Tersenghi, and R. Zecchina. Two solutions to diluted pspin models and XORSAT problems. Journal of Statistical Physics, 111(34):505-533, 2003.

[53] D. G. Mitchell. Resolution complexity of random constraints. In Principles and Practices of Constraint Programming - CP 2002, pages 295-309, 2002.

[54] D. G. Mitchell. Resolution and constraint satisfaction. In Principles and Practices of Constraint Programming - CP 2003, pages 555-569, 2003.

[55] M. Mitzenmacher and E. Upfal. Probability and Computing. Cambridge University Press, 2005.

[56] M. Molloy. Models and thresholds for random constraint satisfaction problems. SIAM Journal of Computing, 32:935-949, 2003.

[57] M. Molloy. Cores in random hypergraphs and boolean formulas. Random Structures and Algorithms, 27(1):124-135, 2005.

[58] M. Molloy. When does the giant component bring unsatisfiability? Combinatorica, 28:693-674, 2008.

[59] M. Molloy and M. Salavatipour. The resolution complexity of random constraint satisfaction problems. SIAM Journal of Computing, 37(3):895922, 2007.

[60] R. Monasson, R. Zecchina, S. Kirkpatrick, B. Selman, and L. Troyansky. $2+p$-SAT: Relation of typical-case complexity to the nature of the phase transition. Random Structures and Algorithms, 15(3-4):414-435, OctoberDecember 1999.

[61] R. E. Moore. Interval Analysis. Prentice-Hall, 1966.

[62] L. Moser and M. Wyman. Stirling numbers of the second kind. Duke Mathematical Journal, 25:29-43, 1958.

[63] B. Pittel, J. Spencer, and N. Wormald. Sudden emergence of a giant $k$-core in a random graph. Journal of Combinatorial Theory, Series B, 67(1):111$151,1996$. 
[64] O. Riordan. The k-core and branching processes. arXiv math/0511093v2 [math.CO], 2007.

[65] The international SAT competitions web page. http://www.satcompetition.org/

[66] SAT07 competition. web page: http://www.cril.univ-artois.fr/SAT07/, 2007. The UE-CSP instances are labeled as connm-ue-csp-sat.

[67] SAT09 competition. web page: http://www.cril.univ-artois.fr/SAT09/, 2009. The UE-CSP instances are labeled as connm-ue-csp-sat.

[68] T. J. Schaefer. The complexity of satisfiability problems. In Proceedings of the Tenth Annual ACM Symposium on Theory of Computing, pages 216226, 1978.

[69] B. Selman, D. G. Mitchell, and H. J. Levesque. Generating hard satisfiability problems. Artificial Intelligence, 81(1-2):17-29, 1996.

[70] B. M. Smith. Constructing an asymptotic phase transition in random binary constraint satisfaction problems. Theoretical Computer Science, 265(1-2):265-283, August 2001.

[71] N. M. Temme. Asymptotic estimates of stirling numbers. Studies in Applied Mathematics, 89:233-243, 1993.

[72] K. Xu and W. Li. Exact phase transitions in random constraint satisfaction problems. Journal of Artificial Intelligence Research, 12:93-103, 2000.

[73] K. Xu and W. Li. Many hard examples in exact phase transitions. Theoretical Computer Science, 355(3):291-302, 2006.

\section{A The Proof that $f$ has a unique global maxi- mum.}

In this appendix, we present the computer-aided proof of Lemma 18, which we restate below. Let

$$
\begin{aligned}
f(\alpha, r, t)= & \ln 4-c \ln 4+(1-\alpha) \ln 3-c(2+t-3 r) \ln 3 \\
& +c(1-3 r+2 t) \ln 2-\alpha \ln \alpha-(1-\alpha) \ln (1-\alpha) \\
& -c(1-3 r+2 t) \ln (1-3 r+2 t)-c(3 r-3 t) \ln (r-t)-c t \ln t \\
& +r 3 c \ln r+(1-r) 3 c \ln (1-r)+\alpha \ln \left(\mathrm{e}^{z}-1-z\right)-r 3 c \ln z \\
& +(1-\alpha) \ln \left(\mathrm{e}^{y}-1-y\right)-(1-r) 3 c \ln y-\ln \left(\mathrm{e}^{x}-1-x\right) \\
& +3 c \ln x,
\end{aligned}
$$

where $x, y, z>0$ are defined as

$$
\frac{\mathrm{e}^{x}-1-x}{\mathrm{e}^{x}-1}-\frac{x}{3 c}=\frac{\mathrm{e}^{y}-1-y}{\mathrm{e}^{y}-1}-\frac{y(1-\alpha)}{3 c(1-r)}=\frac{\mathrm{e}^{z}-1-z}{\mathrm{e}^{z}-1}-\frac{z \alpha}{3 c r}=0 .
$$


Lemma 18 For all $\frac{2}{3} \leq c<1$, the unique global maximum of $f(\alpha, r, t)$ in the domain $0<\alpha<1, \frac{2 \alpha}{3 c}<r<1-\frac{2(1-\alpha)}{3 c}$, and $\max \left\{0, \frac{3 r-1}{2}\right\}<t<r$ occurs at $f\left(\frac{1}{4}, \frac{1}{4}, \frac{1}{16}\right)=2(1-c) \ln 4$. Furthermore, for all .67 $\leq c \leq 1$, there exists a constant $b=b(c)$ such that at every extremal $(\alpha, r, t), f$ approaches a limit that is less than $2(1-c) \ln 4-b$.

Proof Recall from Observation 17 that $f$ is continuous and differentiable throughout the domain $0<\alpha<1, \frac{2 \alpha}{3 c}<r<1-\frac{2(1-\alpha)}{3 c}, \max \left\{0, \frac{3 r-1}{2}\right\}<$ $t<r$. In Section 5, we proved that (i) $f$ has one local maximum when $z=y$, (ii) the point $\alpha=\frac{1}{4}, r=\frac{1}{4}, t=\frac{1}{16}$ is this local maximum, and (iii)

$$
f\left(\frac{1}{4}, \frac{1}{4}, \frac{1}{16}\right)=2(1-c) \ln 4 .
$$

Lemma 25 in Section A.1 gives an equation in terms of $z$ and $y$ for all points where the partial first derivatives of $f$ are 0. Lemma 26 of Section A.3 proves that at each point in the domain of $f$ which satisfies both the equation of Lemma 25 and $z \neq y$, the value of $f$ at that point is smaller than 2(1c) $\ln 4$. To complete the proof, Lemma 32 of Section A.4 proves that at every point $\left(\alpha_{1}, r_{1}, t_{1}\right)$ on the boundary of the domain of $f$ (i.e. for every extremal $\left.\left(\alpha_{1}, r_{1}, t_{1}\right)\right), \lim _{(\alpha, r, t) \rightarrow\left(\alpha_{1}, r_{1}, t_{1}\right)} f(\alpha, r, t)$ exists and is less than $2(1-c) \ln 4-b$ where $b>0$ is a constant independent of $\alpha_{1}, r_{1}$, and $t_{1}$, but it may be dependent of $c$.

\section{A.1 An Equation for All Stationary Points of $f$}

We start by relating $z$ and $y$ at all stationary points.

Lemma 25 Every stationary point of the function $f$ of Lemma 18 satisfies the equation

$$
\frac{z}{y}=\frac{3\left(\mathrm{e}^{y}-1\right)^{2}+\left(\mathrm{e}^{z}-1\right)^{2}}{2\left(\mathrm{e}^{y}-1\right)^{2}+2\left(\mathrm{e}^{y}-1\right)\left(\mathrm{e}^{z}-1\right)} .
$$

Furthermore, for each $y>0$, there are at exactly two values for $z$ that satisfy 24), one when $z=y$ and one when $z>y$.

Proof To find all stationary points for $f$ in the interior of the domain, we use the partial derivatives of $f$, calculated in Section [5, and from these we know that any stationary point must satisfy all of the following equations.

$$
\begin{aligned}
\frac{1-\alpha}{\alpha} & =3 \frac{\mathrm{e}^{y}-1-y}{\mathrm{e}^{z}-1-z} \\
\frac{1-r}{r} & =\frac{y}{z} \cdot \frac{3}{2} \cdot \frac{(1-3 r+2 t)}{(r-t)} \\
\frac{(r-t)^{3}}{(1-3 r+2 t)^{2}} & =\frac{3 t}{4}
\end{aligned}
$$


From (22), we have

$$
\frac{\mathrm{e}^{y}-1-y}{\mathrm{e}^{z}-1-z}=\frac{y}{z} \cdot \frac{(1-\alpha)}{\alpha} \cdot \frac{r}{(1-r)} \cdot \frac{\left(\mathrm{e}^{y}-1\right)}{\left(\mathrm{e}^{z}-1\right)} .
$$

Plugging in (25), gives

$$
3 \frac{y}{z} \cdot \frac{r}{(1-r)}=\frac{\mathrm{e}^{z}-1}{\mathrm{e}^{y}-1},
$$

and combining (28) with (26) gives

$$
\frac{2(r-t)}{1-3 r+2 t}=\frac{\mathrm{e}^{z}-1}{\mathrm{e}^{y}-1}
$$

Combining (27) with (29) gives

$$
\left(\frac{\mathrm{e}^{z}-1}{\mathrm{e}^{y}-1}\right)^{2}(r-t)=3 t .
$$

Solving (26) for $t$ yields

$$
t=\frac{2 z r(1-r)-3 y r+9 y r^{2}}{6 y r+2 z(1-r)},
$$

and plugging this value for $t$ into (30) gives

$$
\left(\frac{\mathrm{e}^{z}-1}{\mathrm{e}^{y}-1}\right)^{2} y(1-r)=2 z(1-r)-3 y+9 y r .
$$

Solving (28) for $r$ gives

$$
r=\frac{\left(\mathrm{e}^{z}-1\right) z}{3\left(\mathrm{e}^{y}-1\right) y+\left(\mathrm{e}^{z}-1\right) z},
$$

and substituting (32) for $r$ in (31) gives

$$
\frac{z}{y}=\frac{3\left(\mathrm{e}^{y}-1\right)^{2}+\left(\mathrm{e}^{z}-1\right)^{2}}{2\left(\mathrm{e}^{y}-1\right)^{2}+2\left(\mathrm{e}^{y}-1\right)\left(\mathrm{e}^{z}-1\right)} .
$$

This establishes (24). To prove the rest of the lemma, we will look at cases.

First, we can rule out the case when $0<z<y$. If $0<z<y$ then the left hand side of (24) is smaller than 1, but it is straightforward to see that the right hand side is equal to $1+\frac{\left(\mathrm{e}^{y}-\mathrm{e}^{z}\right)^{2}}{2\left(\mathrm{e}^{y}-1\right)^{2}+2\left(\mathrm{e}^{y}-1\right)\left(\mathrm{e}^{z}-1\right)}$ and so is always at least 1 , with equality when $z=y$.

Now we prove that for each $y>0$, there is exactly one solution with $z>y$. We will do this by fixing $y$ and determining how each side of equation (24) changes as $z$ increases. We will calculate the first and second derivatives, with respect to $z$, of the right hand side of (24), and we will note that the first derivative is 0 when $z=y$, both derivatives are positive when $z>y$, and the 
first derivative grows unbounded as $z$ tends to infinity and $y$ is fixed. That implies that, for any fixed $y$, the right hand side of (24) will cross $\frac{z}{y}$ exactly once when $z>y$.

The first derivative of the RHS of (24) with respect to $z$ is

$$
\frac{2 \mathrm{e}^{z}\left(\mathrm{e}^{y}-1\right)\left(\left(2\left(\mathrm{e}^{y}-1\right)+2\left(\mathrm{e}^{z}-1\right)\right)\left(\mathrm{e}^{z}-1\right)-\left(3\left(\mathrm{e}^{y}-1\right)^{2}+\left(\mathrm{e}^{z}-1\right)^{2}\right)\right)}{\left(2\left(\mathrm{e}^{y}-1\right)^{2}+2\left(\mathrm{e}^{y}-1\right)\left(\mathrm{e}^{z}-1\right)\right)^{2}}
$$

which can be rewritten as

$$
\frac{\mathrm{e}^{z}\left(\mathrm{e}^{z}-\mathrm{e}^{y}\right)\left(\mathrm{e}^{z}-1+3\left(\mathrm{e}^{y}-1\right)\right)}{2\left(\mathrm{e}^{y}-1\right)\left(\mathrm{e}^{z}+\mathrm{e}^{y}-2\right)^{2}} .
$$

It is straightforward to see that (33) is negative when $0<z<y$, is positive when $z>y>0$, and grows unbounded as $z>0$ increases.

The second derivative of the RHS of (24) with respect to $z$ is

$$
\begin{array}{r}
\frac{\mathrm{e}^{z}}{2\left(\mathrm{e}^{y}-1\right)\left(\mathrm{e}^{z}+\mathrm{e}^{y}-2\right)^{3}}\left(( \mathrm { e } ^ { z } + \mathrm { e } ^ { y } - 2 ) \left[\left(\mathrm{e}^{z}-\mathrm{e}^{y}\right)\left(\mathrm{e}^{z}-1+3\left(\mathrm{e}^{y}-1\right)\right)\right.\right. \\
\left.+\mathrm{e}^{z}\left(\mathrm{e}^{z}-1+3\left(\mathrm{e}^{y}-1\right)\right)+\mathrm{e}^{z}\left(\mathrm{e}^{z}-\mathrm{e}^{y}\right)\right] \\
\left.-2 \mathrm{e}^{z}\left(\mathrm{e}^{z}-\mathrm{e}^{y}\right)\left(\mathrm{e}^{z}-1+3\left(\mathrm{e}^{y}-1\right)\right)\right)
\end{array}
$$

which simplifies to

$$
\frac{\mathrm{e}^{z}\left(\left(\mathrm{e}^{z}+\mathrm{e}^{y}-2\right)\left(\mathrm{e}^{z}-\mathrm{e}^{y}\right)\left(\mathrm{e}^{z}-1+3\left(\mathrm{e}^{y}-1\right)\right)+8 \mathrm{e}^{z}\left(\mathrm{e}^{y}-1\right)^{2}\right)}{2\left(\mathrm{e}^{y}-1\right)\left(\mathrm{e}^{z}+\mathrm{e}^{y}-2\right)} .
$$

It is straightforward to verify that (34) is positive when $z>y>0$.

\section{A.2 Interval Analysis}

The technique of interval analysis 61, 38 is a method to rigorously bound the range of values that a function takes over an interval. If the endpoints of the interval are also rigorously determined, then we have a proven upper and lower bound on that range. Interval analysis is very useful with numerical algorithms because we can include all errors of floating point approximation into the interval bounds. Following standard interval analysis notation, we denote an interval with a capital letter; eg., $X=[\underline{x}, \bar{x}]$ is an interval where $\underline{x}$ and $\bar{x}$ are real numbers and denote the endpoints of the interval.

If we let $A=[\underline{\alpha}, \bar{\alpha}], R=[\underline{r}, \bar{r}]$, and $T=[\underline{t}, \bar{t}]$ be intervals, we can use interval analysis techniques to compute the interval $\Phi=[\underline{\phi}, \bar{\phi}]$ where $\Phi \supseteq\{\phi=$ $f(\alpha, r, t) \mid \alpha \in A, r \in R, t \in T\}$. If we can then prove that $\bar{\phi}<2(1-c) \ln 4$, then we have a proof that $f(\alpha, r, t)<2(1-c) \ln 4$ for all $\alpha \in A, r \in R$, and $t \in T$, as required.

In Section A.3 we prove that there are no global maxima in the interior of the domain, except for the point $\alpha=\frac{1}{4}, r=\frac{1}{4}, t=\frac{1}{16}$, and in Section A.4 we prove that there are no global maxima on the boundary of the domain. In both cases, the proof relies on an interval analysis program to rule out the various cases. The specifications of the program are listed in Section A.5 


\section{A.3 The Interior of the Domain}

The goal of this section is to rule out all possible stationary points, as defined by (24), with $z>y>0$, from being global maxima.

Lemma 26 For all $\frac{2}{3} \leq c<1$, consider any stationary point of the function $f$ of Lemma 18, in the domain $0<\alpha<1, \frac{2 \alpha}{3 c}<r<1-\frac{2(1-\alpha)}{3 c}$, $\max \left\{0, \frac{3 r-1}{2}\right\}<$ $t<4 r$, and with $z>y$. The maximum value that $f$ can take at this point is smaller than $2(1-c) \ln 4-0.1$.

The proof of Lemma 26 relies on an interval analysis program, and the analysis will be on a slightly different function. We define

$$
\begin{aligned}
F(c, \alpha, r, t, x, y, z)= & \ln 4-c \ln 4+(1-\alpha) \ln 3-c(2+t-3 r) \ln 3 \\
& +c(1-3 r+2 t) \ln 2-\alpha \ln \alpha-(1-\alpha) \ln (1-\alpha) \\
& -c(1-3 r+2 t) \ln (1-3 r+2 t)-c(3 r-3 t) \ln (r-t) \\
& -c t \ln t+r 3 c \ln r+(1-r) 3 c \ln (1-r) \\
& +\Upsilon(x)-\alpha \Upsilon(z)-(1-\alpha) \Upsilon(y)
\end{aligned}
$$

where

$$
\Upsilon(w)=\frac{\left(\mathrm{e}^{w}-1\right) w}{\mathrm{e}^{w}-1-w} \ln w-\ln \left(\mathrm{e}^{w}-1-w\right) .
$$

Note that from (22),

$$
\begin{aligned}
\Upsilon(x) & =3 c \ln x-\ln \left(\mathrm{e}^{x}-1-x\right) \\
(1-\alpha) \Upsilon(y) & =(1-r) 3 c \ln y-(1-\alpha) \ln \left(\mathrm{e}^{y}-1-y\right) \\
\alpha \Upsilon(z) & =r 3 c \ln z-\alpha \ln \left(\mathrm{e}^{z}-1-z\right) .
\end{aligned}
$$

$F$ is defined exactly the same as $f$ except that $x, y$, and $z$ are parameters of $F$ instead of being defined by $\alpha$ and $r$, and $c$ is an explicit parameter of $F$. Given intervals for each of the parameters of $F$, we will determine an interval

$$
\begin{array}{r}
{[\phi, \bar{\phi}] \supseteq\{\phi \mid \phi=F(c, \alpha, r, t, x, y, z) \text { for } c \in[\underline{c}, \bar{c}], \alpha \in[\underline{\alpha}, \bar{\alpha}], r \in[\underline{r}, \bar{r}],} \\
t \in[\underline{t}, \bar{t}], x \in[\underline{x}, \bar{x}], y \in[\underline{y}, \bar{y}], \text { and } z \in[\underline{z}, \bar{z}]\} .
\end{array}
$$

Note that if $[\underline{x}, \bar{x}],[\underline{y}, \bar{y}]$, and $[\underline{z}, \bar{z}]$ contain all values of $x, y, z>0$ that satisfy (22) for each $c \in[\underline{c}, \overline{\bar{c}}], \alpha \in[\underline{\alpha}, \bar{\alpha}]$ and $r \in[\underline{r}, \bar{r}]$ then

$$
\begin{gathered}
{[\underline{\phi}, \bar{\phi}] \supseteq\{\phi \mid \phi=f(\alpha, r, t) \text { for } \alpha \in[\underline{\alpha}, \bar{\alpha}], r \in[\underline{r}, \bar{r}], \text { and } t \in[\underline{t}, \bar{t}]} \\
\text { and where } \underline{c} \leq c \leq \bar{c}\} .
\end{gathered}
$$

For a certain constant $b$, we verify for every such interval, we have $2(1-\underline{c}) \ln 4-$ $\bar{\phi} \geq b$. This proves that $f(\alpha, r, t)<2(1-c) \ln 4-b$ for all $\alpha \in[\underline{\alpha}, \bar{\alpha}], r \in[\underline{r}, \bar{r}]$, $t \in[\underline{t}, \bar{t}]$ and where $\underline{c} \leq c \leq \bar{c}$. Our interval analysis program proves that such a $b$ exists and its value is between 0.010 and 0.055 .

The interval analysis is as follows. First, we will place an upper bound on the value of $y$ at any stationary point with $z>y$ and with $\frac{2}{3} \leq c<1$. We define: 
Definition $27 x^{*}$ is the largest solution to $\frac{x\left(\mathrm{e}^{x}-1\right)}{\mathrm{e}^{\mathrm{x}}-1-\mathrm{x}}=3$.

From (22), it is straightforward to show that if $z>y$, then $z>x>y$, and if $c<1$ then $x$, and by extension $y$, cannot exceed $x^{*}$.

While $y$ is defined to be larger than 0 , we note that, from Observation 16. the value of $f$ at the domain boundary $r=\frac{2 \alpha}{3 c}$ is the limit of $f$ as $y$ approaches 0 , and from Observation 17, the limit of $f$ at the border exists. As a result, we have to rule out the case that the value of $f$ exceeds $2(1-c) \ln 4$ at any point where $y$ is arbitrarily close to 0 . To do so, we will extend the interval $\left(0, x^{*}\right)$ of possible values for $y$ to $\left[0, x^{*}\right]$. We can rewrite (24) as

$$
2 z=\frac{3\left(\mathrm{e}^{y}-1\right)^{2}+\left(\mathrm{e}^{z}-1\right)^{2}}{\mathrm{e}^{y}-1+\mathrm{e}^{z}-1} \cdot \frac{y}{\mathrm{e}^{y}-1}
$$

and we note that as $y$ tends to $0, z$ tends to the largest solution of

$$
2 z=\mathrm{e}^{z}-1
$$

We will cover the interval $\left[0, x^{*}\right]$ of possible values for $y$ with overlapping subintervals. For each subinterval $Y=[\underline{y}, \bar{y}]$, we will compute intervals that contain all possible values that $z, x, c, \alpha, r$, and $t$ can take at an stationary point of $f$ with $y \in Y$ and $y<z$. We will then compute the maximum value that $F$ can take on these intervals and verify that this value is smaller than $2(1-c) \ln 4$.

Given $Y=[y, \bar{y}]$, we first compute an interval that contains all $z$ such that: $Y$ contains at least one $y<z$ where $(z, y)$ satisfies (24) of Lemma 25. Let the function $z(y)$ be defined as the largest solution to (24) when $y>0$ and as the largest solution to (36) when $y=0$. Given the interval $Y=[\underline{y}, \bar{y}]$, we find an interval $Z=[\underline{z}, \bar{z}]$ such that

$$
[\underline{z}, \bar{z}] \supseteq\{z \mid z=z(y) \text { for } y \in[\underline{y}, \bar{y}]\}
$$

Because $z$ is only defined implicitly in (24) and (36), we do the following to compute $[\underline{z}, \bar{z}]$.

Let $z_{1}=z(\underline{y})$, and let $z_{2}=z(\bar{y})$. Using the bisection method, we find intervals $Z_{1}=\left[\underline{z_{1}}, \overline{z_{1}}\right]$ and $Z_{2}=\left[\underline{z_{2}}, \overline{z_{2}}\right]$ that contain $z_{1}$ and $z_{2}$, respectively. We do not use $\left[\underline{z}_{1}, \overline{z_{2}}\right]$ as the interval $Z$ because to do so, we would need to know that $\frac{d z}{d y}>0$. A proof of this inequality appears to be challenging, so instead we will find a $\delta$ and $\delta^{\prime}$ such that $Z=\left[\underline{z_{1}}-\delta^{\prime}, \overline{z_{2}}+\delta\right]$ satisfies (37).

From (24), Let

$$
\lambda(y, z)=y\left(\frac{3\left(\mathrm{e}^{y}-1\right)^{2}+\left(\mathrm{e}^{z}-1\right)^{2}}{2\left(\mathrm{e}^{y}-1\right)^{2}+2\left(\mathrm{e}^{y}-1\right)\left(\mathrm{e}^{z}-1\right)}\right),
$$

and so for $y>0, z(y)$ is the largest solution to $z=\lambda(y, z)$. Let

$$
\lambda_{I}(Y, Z)=\{\lambda(y, z) \mid y \in Y, z \in Z\} .
$$


From our computation of $Z_{1}$ and $Z_{2}$, we know that there is a $z \in\left[\underline{z_{1}}, \overline{z_{2}}\right]$ such that $z=\lambda(y, z)$ for some $y \in Y$ (eg. $z=\underline{z_{1}}$ and $\left.z=\overline{z_{2}}\right)$. This implies $z \in \lambda_{I}\left(Y,\left[\underline{z_{1}}, \overline{z_{2}}\right]\right)$, and

$$
\left[\underline{z_{1}}, \overline{z_{2}}\right] \cap \lambda_{I}\left(Y,\left[\underline{z_{1}}, \overline{z_{2}}\right]\right) \neq \emptyset \text {. }
$$

To compute bounds for $Z$, we will find $\delta, \delta^{\prime}, \epsilon>0$ such that

$$
\begin{aligned}
{\left[\underline{z_{1}}-\delta^{\prime}-\epsilon, \underline{z_{1}}-\delta^{\prime}\right] \cap \lambda_{I}\left(Y,\left[\underline{z_{1}}-\delta^{\prime}-\epsilon, \underline{z_{1}}-\delta^{\prime}\right]\right) } & =\emptyset \\
{\left[\overline{z_{2}}+\delta, \overline{z_{2}}+\delta+\epsilon\right] \cap \lambda_{I}\left(Y,\left[\overline{z_{2}}+\delta, \overline{z_{2}}+\delta+\epsilon\right]\right) } & =\emptyset .
\end{aligned}
$$

We will prove that for every $y \in Y, \overline{z_{2}}+\delta$ is an upper bound on the largest $z$ such that $z=\lambda(y, z)$. Suppose that for some $y^{\prime} \in Y$, there is a $z^{\prime}>\overline{z_{2}}+\delta$ such that $z^{\prime}=\lambda\left(y^{\prime}, z^{\prime}\right)$. From the definition of $\lambda$, we have $z\left(y^{\prime}\right)=z^{\prime}$. Because $z(\cdot)$ is continuous, the intermediate value theorem states that for every $z_{1}<z^{*}<z^{\prime}$ there is some $y^{*} \in\left[\underline{y}, y^{\prime}\right] \subset Y$ with $z\left(y^{*}\right)=z^{*}$, and so $z^{*}=\lambda\left(y^{*}, z^{*}\right)$. Picking $z_{2}+\delta<z^{*}<\min \left(z_{2}+\delta+\epsilon, z^{\prime}\right)$ yields a contradiction to the statement

$$
\left[\overline{z_{2}}+\delta, \overline{z_{2}}+\delta+\epsilon\right] \cap \lambda_{I}\left(Y,\left[\overline{z_{2}}+\delta, \overline{z_{2}}+\delta+\epsilon\right]\right)=\emptyset
$$

A similar argument shows that for every $y \in Y, \underline{z_{1}}-\delta^{\prime}$ is a lower bound on the smallest $z$ such that $z=\lambda(y, z)$.

Therefore, we can set $Z=\left[z_{1}-\delta^{\prime}, \overline{z_{2}}+\delta\right]$. To find appropriate $\delta$ and $\delta^{\prime}$ values, we set $\epsilon=2.22 \times 10^{-16}$, and we use binary search to find small $\delta$ and $\delta^{\prime}$ that satisfy the above properties.

Next, we combine (22) with the equations (25) and (26) that must hold at any stationary point of $f$ to define the following function,

$$
c(y, z)=\frac{3\left(\mathrm{e}^{y}-1\right) y+\left(\mathrm{e}^{z}-1\right) z}{3 \times\left(3\left(\mathrm{e}^{y}-1-y\right)+\mathrm{e}^{z}-1-z\right)},
$$

and given the intervals $[\underline{y}, \bar{y}]$ and $[\underline{z}, \bar{z}]$, we use standard interval operations to compute

$$
[\underline{c}, \bar{c}] \supseteq\{c \mid c=c(y, z) \text { for } y \in[\underline{y}, \bar{y}] \text { and } z \in[\underline{z}, \bar{z}]\} .
$$

From (22), we define the function $x(c)$ as the largest solution to

$$
3 c=\frac{x\left(\mathrm{e}^{x}-1\right)}{\mathrm{e}^{x}-1-x} .
$$

and we use $x(c)$ to find an interval $[\underline{x}, \bar{x}]$ given the interval $[\underline{c}, \bar{c}]$. Let

$$
[\underline{x}, \bar{x}] \supseteq\{x \mid x=x(c) \text { for } c \in[\underline{c}, \bar{c}]\} .
$$

Because $x$ is only defined implicitly in (38), we use the following procedure to compute $X=[\underline{x}, \bar{x}]$.

It is straightforward to verify the $\frac{x\left(\mathrm{e}^{x}-1\right)}{\mathrm{e}^{x}-1-x}$ is an increasing function when $x>0$. As a result, (38) will have at most one positive solution. Given $[\underline{c}, \bar{c}]$, we use Lemma 28 below to give positive upper and lower bounds for both $x(\underline{c})$ 
and $x(\bar{c})$, and then we can use binary search, starting from these bounds, to find the intervals $X_{1}=\left[\underline{x_{1}}, \overline{x_{1}}\right]$ and $X_{2}=\left[\underline{x_{2}}, \overline{x_{2}}\right]$ that contain $x(\underline{c})$ and $x(\bar{c})$, respectively. From (38), it is straightforward to see that $\frac{d x}{d c}>0$, and so we can set $X=\left[\underline{x_{1}}, \overline{x_{2}}\right]$.

Lemma 28 Let $3 c=\frac{x\left(\mathrm{e}^{x}-1\right)}{\mathrm{e}^{x}-1-x}$ and $c \geq \frac{2}{3}$. If $x>0$ then $3 c-2<x<3 c$.

Proof First note that $\lim _{x \rightarrow 0} \frac{x\left(\mathrm{e}^{x}-1\right)}{\mathrm{e}^{x}-1-x}=2$. So when $x$ approaches $0, c$ approaches $\frac{2}{3}$, and we have, in the limit, $x+2=3 c$. Next note that $x<x\left(\frac{\mathrm{e}^{x}-1}{\mathrm{e}^{x}-1-x}\right)=3 c$. To complete the proof, we show that if $x>0$, the derivative of $\frac{x\left(\mathrm{e}^{x}-1\right)}{\mathrm{e}^{x}-1-x}$ is less than 1 . This implies that for $x>0,3 c$ is sandwiched between $x$ and $x+2$.

The derivative of $\frac{x\left(\mathrm{e}^{x}-1\right)}{\mathrm{e}^{x}-1-x}$ is $\frac{\left(\mathrm{e}^{x}-1\right)^{2}-x^{2} \mathrm{e}^{x}}{\left(\mathrm{e}^{x}-1-x\right)^{2}}$. To show that this derivative is less than 1 , we show

$$
\begin{aligned}
\left(\mathrm{e}^{x}-1-x\right)^{2}-\left(\mathrm{e}^{x}-1\right)^{2}+x^{2} \mathrm{e}^{x} & =-2 x\left(\mathrm{e}^{x}-1\right)+x^{2}+x^{2} \mathrm{e}^{x} \\
& =x\left(-2\left(\mathrm{e}^{x}-1\right)+x+x \mathrm{e}^{x}\right) \\
& \geq 0
\end{aligned}
$$

The last inequality follows by noting that the first and second derivatives of $-2\left(\mathrm{e}^{x}-1\right)+x+x \mathrm{e}^{x}$ are $x \mathrm{e}^{x}-\mathrm{e}^{x}+1$ and $x \mathrm{e}^{x}$, respectively.

It is now straightforward to use equations that must hold at any stationary point of $f$ to define intervals for the remaining parameters of $F$ given the intervals $[\underline{y}, \bar{y}]$ and $[\underline{z}, \bar{z}]$. We use $(25)$ to define the function

$$
\alpha(y, z)=\frac{\mathrm{e}^{z}-1-z}{3\left(\mathrm{e}^{y}-1-y\right)+\mathrm{e}^{z}-1-z},
$$

and given the intervals $[\underline{y}, \bar{y}]$ and $[\underline{z}, \bar{z}]$, we use standard interval operations to compute the interval

$$
[\underline{\alpha}, \bar{\alpha}] \supseteq\{\alpha \mid \alpha=\alpha(y, z) \text { for } y \in[\underline{y}, \bar{y}] \text { and } z \in[\underline{z}, \bar{z}]\} .
$$

We use (32) to define the function

$$
r(y, z)=\frac{\left(\mathrm{e}^{z}-1\right) z}{3\left(\mathrm{e}^{y}-1\right) y+\left(\mathrm{e}^{z}-1\right) z},
$$

and given the intervals $[\underline{y}, \bar{y}]$ and $[\underline{z}, \bar{z}]$, we use standard interval operations to compute the interval

$$
[\underline{r}, \bar{r}] \supseteq\{r \mid r=r(y, z) \text { for } y \in[\underline{y}, \bar{y}] \text { and } z \in[\underline{z}, \bar{z}]\} .
$$

We use (30) to define the function

$$
t(r, y, z)=\frac{\left(\mathrm{e}^{z}-1\right)^{2} r}{3\left(\mathrm{e}^{y}-1\right)^{2}+\left(\mathrm{e}^{z}-1\right)^{2}},
$$


and given the intervals $[\underline{r}, \bar{r}],[\underline{y}, \bar{y}]$ and $[\underline{z}, \bar{z}]$, we use standard interval operations to compute the interval

$$
[\underline{t}, \bar{t}] \supseteq\{t \mid t=t(r, y, z) \text { for } r \in[\underline{r}, \bar{r}], y \in[\underline{y}, \bar{y}] \text { and } z \in[\underline{z}, \bar{z}]\} .
$$

Finally, we use standard interval operations to compute the interval

$$
\begin{array}{r}
{[\underline{\phi}, \bar{\phi}]=\{\phi \mid \phi=F(c, \alpha, r, t, x, y, z) \text { for } c \in[\underline{c}, \bar{c}], \alpha \in[\underline{\alpha}, \bar{\alpha}], r \in[\underline{r}, \bar{r}],} \\
t \in[\underline{t}, \bar{t}], x \in[\underline{x}, \bar{x}], y \in[\underline{y}, \bar{y}], \text { and } z \in[\underline{z}, \bar{z}]\} .
\end{array}
$$

We then verify that $\bar{\phi}<2(1-\bar{c}) \ln 4$, and this verifies that at every point in the given intervals the value of $F$, and therefore $f$, is smaller than $2(1-c) \ln 4$. Repeating this process for every overlapping subinterval $[y, \bar{y}] \subset\left[0, x^{*}\right]$, the program proves that there exists a constant $b$ with $0.010<\bar{b}<0.055$ such that that the value for $f$ on each interval is smaller than $2(1-c) \ln 4-b$, and this proves Lemma 26.

These computations are all performed by an interval analysis program specified in Section A.5. The computation of the interval $[\phi, \bar{\phi}]$ uses standard interval analysis techniques plus the following observations.

Observation 29 The function $\Upsilon(w)$ has the following form. When $w \rightarrow 0$, the function approaches $\ln 2$, the function has one minimum at $w=1$ with value $-\ln (e-2)$, and the function grows unbounded as $w \rightarrow \infty$.

In the cases that $z$ becomes large, we can use the following approximations for $\Upsilon(z)$. These approximations have reduced dependency errors and will, for large $z$, result in a tighter bound than a computation of $\Upsilon(z)$.

\section{Observation 30}

$$
\Upsilon(w)=\frac{\left(\mathrm{e}^{w}-1\right) w}{\mathrm{e}^{w}-1-w} \ln w-\ln \left(e^{w}-1-w\right) \geq w(\ln w-1) .
$$

Observation 31 For $w>2.5$,

$$
\Upsilon(w)=\frac{\left(\mathrm{e}^{w}-1\right) w}{\mathrm{e}^{w}-1-w} \ln w-\ln \left(\mathrm{e}^{w}-1-w\right) \leq w(\ln w-1)+1 .
$$

\section{A.4 The Boundary of the Domain}

The final step required for the proof of Lemma 18 is to prove that at every point on the boundary of the domain, $f$ approaches a limit that is less than $2(1-c) \ln 4$.

Lemma 32 Let $.67 \leq c<1$ and let $b=b(c)$ be a positive constant that depends on $c$ but is independent of $\alpha, r$, and $t$. For every point $\left(\alpha_{1}, r_{1}, t_{1}\right)$ on the boundary of the domain of $f, \lim _{(\alpha, r, t) \rightarrow\left(\alpha_{1}, r_{1}, t_{1}\right)} f(\alpha, r, t)$ exists and is less than $2(1-c) \ln 4-b$. 
The proof of Lemma 32 is the subject of the remainder of this appendix. From Observation 17 the limit exists. The boundary of $f$ consists of 7 faces, the faces intersect at 13 line segments, and the line segments intersect at 8 points.

For each of the 8 points, we take the limit of $f$ as its parameters approach that point and prove that the limit is smaller than $2(1-c) \ln 4$. For each of the line segments, we determine a function on the line segment which is either the limit of $f$ as its parameters approach each point of that boundary or an upper bound on the limit. For the present discussion, we use $f_{B}$ to denote that function. $f_{B}$ will not have a discontinuity, and so any maximum value of $f_{B}$ will occur either at the endpoints of the line segment or at a point where the derivative of $f_{B}$ is 0 . As we have already proven that the endpoints are not global maxima, we can restrict the analysis to the places where the derivative is 0 . Similarly, for each of the 7 faces, we define a similar function $f_{B}$ on the face and evaluate the points where both the partial first derivatives of $f_{B}$ are 0 .

The proof of Lemma 32 will also rely on an interval analysis program. For each boundary, the interval analysis will be performed on the function $F_{B}(c, \alpha, r, t, x, y, z)$ where $F_{B}$ is defined the same as $f_{B}$ except that $x, y$, and $z$ are parameters to $F_{B}$ instead of being defined in terms of $\alpha$ and $r$, and $c$ is an explicit parameter of $F_{B}$. The interval analysis program will verify that for each $c \in[.67,1)$, for each $\alpha, r$, and $t$ in the domain of $f_{B}$, and for each $x, y$, and $z$ such that $x, y, z>0$ and (22) is satisfied, the maximum value for $F_{B}(c, \alpha, r, t, x, y, z)$ is smaller than $2(1-c) \ln 4$, and the program will report the smallest difference between $2(1-c) \ln 4$ and the maximum value for an end point or stationary point on the boundary. Note that when $r=\frac{2 \alpha}{3 c}$, we replace any term of $F_{B}$ involving $z$ with its limit as $z$ tends to 0 . Likewise, when $1-r=\frac{2(1-\alpha)}{3 c}$, we replace any term involving $y$ with its limit as $y$ tends to 0 . From Observation 16 and Observation 17, the limit for $f$ exists at those points. (While we can evaluate $F_{B}$ when $c=\frac{2}{3}$ by replacing any term involving $x$ with its limit as $x$ tends to 0 , this approach will run into problem with Cases 24 and 25 below. Specifically, when $c=\frac{2}{3}$, then the entire domain including the global maximum lies on the boundary $r=\frac{2 \alpha}{3 c}=1-\frac{2(1-\alpha)}{3 c}$.)

The program will cover the interval $[.67,1]$ with overlapping subintervals. Likewise, for each parameter, $\alpha, r$, and $t$, not restricted by the boundary to a single value, the program will cover the legal values of each parameter with overlapping subintervals. Given the intervals $[\underline{c}, \bar{c}],[\underline{\alpha}, \bar{\alpha}]$, and $[\underline{r}, \bar{r}]$, we compute intervals containing the possible values for $x, y$, and $z$, if needed. As is done in Section A.3. we define the function $x(c)$ as the largest solution to

$$
3 c=\frac{x\left(\mathrm{e}^{x}-1\right)}{\mathrm{e}^{x}-1-x},
$$

we define the function $y(c, \alpha, r)$ as the largest solution to

$$
\frac{3 c(1-r)}{1-\alpha}=\frac{y\left(\mathrm{e}^{y}-1\right)}{\mathrm{e}^{y}-1-y},
$$


and we define the function $z(c, \alpha, r)$ as the largest solution to

$$
\frac{3 c r}{\alpha}=\frac{z\left(\mathrm{e}^{z}-1\right)}{\mathrm{e}^{z}-1-z} .
$$

Let

$$
\begin{array}{ll}
{[\underline{x}, \bar{x}]} & \supseteq\{x \mid x=x(c) \text { for } c \in[\underline{c}, \bar{c}]\}, \\
{[\underline{y}, \bar{y}]} & \supseteq\{y \mid y=y(c, \alpha, r) \text { for } c \in[\underline{c}, \bar{c}], \alpha \in[\underline{\alpha}, \bar{\alpha}], \text { and } r \in[\underline{r}, \bar{r}]\}, \text { and } \\
{[\underline{z}, \bar{z}]} & \supseteq\{z \mid z=z(c, \alpha, r) \text { for } c \in[\underline{c}, \bar{c}], \alpha \in[\underline{\alpha}, \bar{\alpha}], \text { and } r \in[\underline{r}, \bar{r}]\} .
\end{array}
$$

Because $x, y$, and $z$ are defined implicitly, we use binary search to separately find upper and lower bounds for the intervals. The technique used to compute $[\underline{x}, \bar{x}]$ is described in Section A.3. and the same technique is used to compute $[\underline{y}, \bar{y}]$ and $[\underline{z}, \bar{z}]$. Lemma 28 gives the initial bounds for $\underline{x}$ and $\bar{x}$, and from the same reasoning we get the following lemmas that give the initial bounds for $\underline{y}$, $\bar{y}, \underline{z}$ and $\bar{z}$.

Lemma 33 Let $\frac{3 c(1-r)}{1-\alpha}=\frac{y\left(\mathrm{e}^{y}-1\right)}{\mathrm{e}^{y}-1-y}, c \geq \frac{2}{3}$, and $\alpha<1$. If $y>0$ then $\frac{3 c(1-r)}{1-\alpha}-$ $2<y<\frac{3 c(1-r)}{1-\alpha}$.

Lemma 34 Let $\frac{3 c r}{\alpha}=\frac{z\left(\mathrm{e}^{z}-1\right)}{\mathrm{e}^{z}-1-z}, c \geq \frac{2}{3}$, and $\alpha>0$. If $z>0$ then $\frac{3 c r}{\alpha}-2<z<$ $\frac{3 c r}{\alpha}$.

Given $[\underline{c}, \bar{c}],[\underline{\alpha}, \bar{\alpha}],[\underline{r}, \bar{r}],[\underline{t}, \bar{t}],[\underline{x}, \bar{x}],[\underline{y}, \bar{y}]$, and $[\underline{z}, \bar{z}]$, if this is a subinterval of a line or face boundary, the program will compute compute all possible values for the partial first derivatives on these intervals and verify that these possible values contain 0 . The program will then compute an interval that contains all possible values for $F_{B}$ on these intervals, it will verify that the upper bound on this interval is smaller than $2(1-\bar{c}) \ln 4$, and it will record the difference between the maximum possible value for $F_{B}$ and $2(1-\bar{c}) \ln 4$. As a result, we know that $f(\alpha, r, t)<2(1-c) \ln 4-b$ for all $c, \alpha, r$, and $t$ in these intervals. Repeating this process for all subintervals of $\left[\frac{2}{3}, 1\right]$ completes the proof of Lemma 32 . The specifics of the program are listed in Section A.5.

The rest of this section gives the specific computations of $f_{B}$ for each boundary.

\section{A.4.1 The Boundary of the Domain of $f$.}

The domain for $f$ is the region bounded by $\alpha \in(0,1), r \in\left(\frac{2 \alpha}{3 c}, 1-\frac{2(1-\alpha)}{3 c}\right)$, and $t \in\left(\min \left\{0, \frac{3 r-1}{2}\right\}, r\right)$. The boundary of the domain for $f$ has 7 faces:

$$
\begin{aligned}
& \alpha=0 ; \quad \alpha=1 ; \quad r=\frac{2 \alpha}{3 c} ; \quad r=1-\frac{2(1-\alpha)}{3 c} ; \\
& t=0 ; \quad t=\frac{3 r-1}{2} ; \quad t=r
\end{aligned}
$$


the faces intersect at 13 line segments:

$$
\begin{array}{lll}
\alpha=0, t=0 ; & \alpha=0, t=r ; & \alpha=0, r=1-\frac{2(1-\alpha)}{3 c} ; \\
\alpha=1, t=\frac{3 r-1}{2} ; & \alpha=1, t=r ; & \alpha=1, r=\frac{2 \alpha}{3 c} ; \\
r=\frac{2 \alpha}{3 c}, t=r ; & r=\frac{2 \alpha}{3 c}, t=\frac{3 r-1}{2} ; & r=\frac{2 \alpha}{3 c}, t=0 ; \\
r=1-\frac{2(1-\alpha)}{3 c}, t=r ; & r=1-\frac{2(1-\alpha)}{3 c}, t=\frac{3 r-1}{2} ; & r=1-\frac{2(1-\alpha)}{3 c}, t=0 ; \\
t=0=\frac{3 r-1}{2}, & &
\end{array}
$$

and the line segments intersect at 8 points:

$$
\begin{array}{ll}
\alpha=0, r=0, t=0 ; & \alpha=0, r=1-\frac{2}{3 c}, t=0 ; \\
\alpha=0, r=1-\frac{2}{3 c}, t=1-\frac{2}{3 c} ; & \alpha=1, r=1, t=1 ; \\
\alpha=1, r=\frac{2}{3 c}, t=\frac{2-c}{2 c} ; & \alpha=1, r=\frac{2}{3 c}, t=\frac{2}{3 c} ; \\
\alpha=\frac{c}{2}, r=\frac{1}{3}, t=0 ; & \alpha=1-c, r=\frac{1}{3}, t=0 .
\end{array}
$$

As we did in Section A.3, we will define $\Upsilon$ with (35) and use it to rewrite $f$ as

$$
\begin{aligned}
f(\alpha, r, t)= & \ln 4-c \ln 4+(1-\alpha) \ln 3-c(2+t-3 r) \ln 3 \\
& +c(1-3 r+2 t) \ln 2-\alpha \ln \alpha-(1-\alpha) \ln (1-\alpha) \\
& -c(1-3 r+2 t) \ln (1-3 r+2 t)-c(3 r-3 t) \ln (r-t) \\
& -c t \ln t+r 3 c \ln r+(1-r) 3 c \ln (1-r) \\
& +\Upsilon(x)-\alpha \Upsilon(z)-(1-\alpha) \Upsilon(y)
\end{aligned}
$$

where $x, y, z>0$ and (22) holds. The following additional observations are used to both compute the limit of $f$ as its parameters approach a boundary and to give an upper bound on that limit.

From (22), the maximum value $x$ can have in $f$ is the largest solution to

$$
3=\frac{x\left(\mathrm{e}^{x}-1\right)}{\mathrm{e}^{x}-1-x}
$$

or $x=2.1491 \ldots$ As a result, the largest value of $\Upsilon(x)$ with $x>1$ is $.60355 \ldots$. The following observation follows from this fact plus Observation 29.

\section{Observation 35}

$$
\Upsilon(x) \leq \ln 2 .
$$

As discussed in Lemma 28, we have $\lim _{x \rightarrow 0} \frac{x\left(\mathrm{e}^{x}-1\right)}{\mathrm{e}^{x}-1-x}=2$, and $\frac{x\left(\mathrm{e}^{x}-1\right)}{\mathrm{e}^{x}-1-x}$ is an increasing function of $x$. We also have $\lim _{x \rightarrow \infty} \frac{x\left(\mathrm{e}^{x}-1\right)}{\mathrm{e}^{x}-1-x}=\infty$. As a result, from (22), we have that as $\alpha \rightarrow 0, z \rightarrow \infty$, as $\alpha \rightarrow 1, y \rightarrow \infty$, as $r \rightarrow \frac{2 \alpha}{3 c}, z \rightarrow 0$, and as $r \rightarrow 1-\frac{2(1-\alpha)}{3 c}, y \rightarrow 0$. From these facts and Observation 29] we have the following observation. 


\section{Observation 36}

$$
\begin{aligned}
\lim _{\alpha \rightarrow 0} \alpha \Upsilon(z) & \geq 0 \\
\lim _{r \rightarrow \frac{2 \alpha}{3 c}} \alpha \Upsilon(z) & =\alpha \ln 2 \\
\lim _{\alpha \rightarrow 1, r \rightarrow 1} \alpha \Upsilon(z) & =\Upsilon(x) \\
\lim _{\alpha \rightarrow 1}(1-\alpha) \Upsilon(y) & \geq 0 \\
\lim _{r \rightarrow 1-\frac{2(1-\alpha)}{3 c}}(1-\alpha) \Upsilon(y) & =(1-\alpha) \ln 2 \\
\lim _{\alpha \rightarrow 0, r \rightarrow 0}(1-\alpha) \Upsilon(y) & =\Upsilon(x)
\end{aligned}
$$

\section{A.4.2 The Boundary Cases That Are Points}

In each case, we take the limit of $f$ as its parameters approach the boundary point, and we prove that this limit is smaller than $2(1-c) \ln 4$. For the first two cases, the proof is straightforward. For cases $3-6$, we bound this limit by a function of a single variable $c$. These cases can be verified without interval analysis. But because we are using an interval analysis program for the later cases, we will shorten the presentation here by verifying these cases with the program as well. For cases 7 and 8, the bound on the limit of $f$ includes limits of $\Upsilon(x), \Upsilon(y)$ and $\Upsilon(z)$ not covered in Observation 36, In these cases, the interval analysis program will compute bounds on these limits of $\Upsilon$ and use them to verify that the limit of $f$ is always smaller than $2(1-c) \ln 4$.

Case 1: the boundary with $\alpha=0, r=0, t=0$.

Applying (39) and (44), we get the following.

$$
\begin{aligned}
\lim _{\substack{\alpha \rightarrow 0, r \rightarrow 0 \\
t \rightarrow 0}} f(\alpha, r, t)= & \ln 4-c \ln 4+\ln 3-2 c \ln 3+c \ln 2 \\
& +\lim _{\alpha \rightarrow 0, r \rightarrow 0}(\Upsilon(x)-\alpha \Upsilon(z)-(1-\alpha) \Upsilon(y)) \\
\leq & \ln 12-c \ln 18 \\
< & 2(1-c) \ln 4-\ln \frac{4}{3}
\end{aligned}
$$

for $c \in[2 / 3,1)$.

Case 2: the boundary with $\alpha=1, r=1, t=1$.

Applying (42) and (41), we get the following.

$$
\begin{aligned}
\lim _{\substack{\alpha \rightarrow 1, r \rightarrow 1 \\
t \rightarrow 1}} f(\alpha, r, t) & =\ln 4-c \ln 4+\lim _{\alpha \rightarrow 1, r \rightarrow 1}(\Upsilon(x)-\alpha \Upsilon(z)-(1-\alpha) \Upsilon(y)) \\
& \leq \ln 4-c \ln 4 \\
& <2(1-c) \ln 4-b
\end{aligned}
$$

for $c \in[2 / 3,1)$ and where $b=b(c)>0$. 
Case 3: the boundary with $\alpha=0, r=1-\frac{2}{3 c}, t=0$.

Applying (39), (43), and Observation 35, we get the following.

$$
\begin{aligned}
\lim _{\substack{\alpha \rightarrow 0, r \rightarrow 1-\frac{2}{3 c} \\
t \rightarrow 0}} f(\alpha, r, t)= & \ln 4-c \ln 4+\ln 3-(-c+2) \ln 3+(-2 c+2) \ln 2 \\
& -(-2 c+2) \ln \left(-2+\frac{2}{c}\right)-(3 c-2) \ln \left(1-\frac{2}{3 c}\right) \\
& +(3 c-2) \ln \left(1-\frac{2}{3 c}\right)+2 \ln \left(\frac{2}{3 c}\right) \\
& +\lim _{\substack{\alpha \rightarrow 0 \\
r \rightarrow 1-\frac{2}{3 c}}}(\Upsilon(x)-\alpha \Upsilon(z)-(1-\alpha) \Upsilon(y)) \\
\leq & \ln \frac{16}{27}-c \ln \frac{4}{3}-2 c \ln c-2(1-c) \ln (1-c) .
\end{aligned}
$$

The interval analysis program verifies that (45) is no larger than $2(1-c) \ln 4-$ 0.005 , for all $c \in[.67,1)$.

Case 4: the boundary with $\alpha=0, r=1-\frac{2}{3 c}, t=1-\frac{2}{3 c}$.

Applying (39), (43), and Observation 35, we get the following.

$$
\begin{aligned}
\lim _{\substack{\alpha \rightarrow 0, r \rightarrow 1-\frac{2}{3 c} \\
t \rightarrow 1-\frac{2}{3 c}}} f(\alpha, r, t)= & \ln 4-c \ln 4+\ln 3-\frac{4}{3} \ln 3+\frac{2}{3} \ln 2-\frac{2}{3} \ln \frac{2}{3 c} \\
& -\left(c-\frac{2}{3}\right) \ln \left(1-\frac{2}{3 c}\right)+(3 c-2) \ln \left(1-\frac{2}{3 c}\right) \\
& +2 \ln \frac{2}{3 c}+\lim _{\substack{\alpha \rightarrow 0 \\
r \rightarrow 1-\frac{2}{3 c}}}(\Upsilon(x)-\alpha \Upsilon(z)-(1-\alpha) \Upsilon(y)) \\
< & \ln 16-\frac{1}{3} \ln 3-c \ln 36+\frac{2}{3}(3 c-2) \ln (3 c-2) \\
& -2 c \ln c .
\end{aligned}
$$

The interval analysis program verifies that (46) is no larger than $2(1-c) \ln 4-$ 0.046 , for all $c \in[.67,1)$. 
Case 5: the boundary with $\alpha=1, r=\frac{2}{3 c}, t=\frac{2}{3 c}$.

Applying (42), (40), and Observation 35, we get the following.

$$
\begin{aligned}
\lim _{\substack{c \rightarrow 1, r \rightarrow \frac{2}{3 c} \\
t \rightarrow \frac{2}{3 c}}} f(\alpha, r, t)= & \ln 4-c \ln 4-\left(2 c-\frac{4}{3}\right) \ln 3+\left(c-\frac{2}{3}\right) \ln 2 \\
& -\left(c-\frac{2}{3}\right) \ln \left(1-\frac{2}{3 c}\right)-\frac{2}{3} \ln \frac{2}{3 c}+\frac{2}{3} \ln \frac{2}{3} \\
& +(3 c-2) \ln \left(1-\frac{2}{3 c}\right) \\
& +\lim _{\substack{\alpha \rightarrow \frac{1}{3 c} \\
r \rightarrow \frac{2}{3}}}(x(x)-\alpha \Upsilon(z)-(1-\alpha) \Upsilon(y)) \\
\leq & \frac{4}{3} \ln 12-c \ln 162+\frac{2}{3}(3 c-2) \ln (3 c-2) \\
& -2 c \ln c .
\end{aligned}
$$

The interval analysis program verifies that (47) is no larger than $2(1-c) \ln 4-$ 0.147 , for all $c \in[.67,1)$.

Case 6: the boundary with $\alpha=1, r=\frac{2}{3 c}, t=\frac{2-c}{2 c}$.

Applying (42), (40), and Observation 35, we get

$$
\begin{aligned}
\lim _{\substack{\alpha \rightarrow 1, r \rightarrow \frac{2}{3 c} \\
t \rightarrow \frac{2-c}{2 c}}} f(\alpha, r, t)= & \ln 4-c \ln 4-\left(2 c+\frac{2-c}{2}-2\right) \ln 3 \\
& +(c-2+2-c) \ln 2 \\
& -(c-2+2-c) \ln \left(1-\frac{2}{c}+\frac{2-c}{c}\right) \\
& -(2-3(2-c)) \ln \left(\frac{2}{3 c}-\frac{2-c}{2 c}\right) \\
& -\frac{2-c}{2} \ln \frac{2-c}{2 c}+2 \ln \frac{2}{3 c}+(3 c-2) \ln \left(1-\frac{2}{3 c}\right) \\
& +\lim _{\alpha \rightarrow \frac{1}{3 c}}(\Upsilon(x)-\alpha \Upsilon(z)-(1-\alpha) \Upsilon(y)) \\
< & \ln 16-c \ln 54+\frac{1}{2}(3 c-2) \ln (3 c-2) \\
& -\frac{1}{2}(2-c) \ln (2-c)-2 c \ln c .
\end{aligned}
$$

The interval analysis program verifies that (48) is no larger than $2(1-c) \ln 4-$ 0.071 , for all $c \in[.67,1)$. 
Case 7: the boundary with $\alpha=\frac{c}{2}, r=\frac{1}{3}, t=0$.

$$
\begin{aligned}
\lim _{\substack{\alpha \rightarrow \frac{c}{2}, r \rightarrow \frac{1}{3} \\
t \rightarrow 0}} f(\alpha, r, t)= & \ln 4-c \ln 4+\left(1-\frac{c}{2}\right) \ln 3-c \ln 3 \\
& -\frac{c}{2} \ln \frac{c}{2}-\left(1-\frac{c}{2}\right) \ln \left(1-\frac{c}{2}\right) \\
& -c \ln \frac{1}{3}+c \ln \frac{1}{3}+2 c \ln \frac{2}{3} \\
& +\lim _{\alpha \rightarrow \frac{c}{2}, r \rightarrow \frac{1}{3}}(\Upsilon(x)-\alpha \Upsilon(z)-(1-\alpha) \Upsilon(y)) \\
= & \ln 24-c \ln 27-\frac{c}{2} \ln 3-\frac{1}{2}(2-c) \ln (2-c)-\frac{c}{2} \ln c \\
& +\lim _{\alpha \rightarrow \frac{c}{2}, r \rightarrow \frac{1}{3}}(\Upsilon(x)-\alpha \Upsilon(z)-(1-\alpha) \Upsilon(y)) .
\end{aligned}
$$

The interval analysis program verifies that (49) is no larger than $2(1-c) \ln 4-$ 0.265 , for all $c \in[.67,1)$.

Case 8: the boundary with $\alpha=1-c, r=\frac{1}{3}, t=0$.

$$
\begin{aligned}
\lim _{\substack{\alpha \rightarrow 1-c, r \rightarrow \frac{1}{3} \\
t \rightarrow 0}} f(\alpha, r, t)= & \ln 4-c \ln 4+c \ln 3-c \ln 3-(1-c) \ln (1-c) \\
& -c \ln c-c \ln \frac{1}{3}+c \ln \frac{1}{3}+2 c \ln \frac{2}{3} \\
& +\lim _{\substack{\alpha \rightarrow 1-c, r \rightarrow \frac{1}{3} \\
=}}(\Upsilon(x)-\alpha \Upsilon(z)-(1-\alpha) \Upsilon(y)) \\
& \ln 4-c \ln 9-(1-c) \ln (1-c)-c \ln c \\
& +\lim _{\substack{\alpha \rightarrow 1-c \\
r \rightarrow \frac{1}{3}}}(\Upsilon(x)-\alpha \Upsilon(z)-(1-\alpha) \Upsilon(y)) .
\end{aligned}
$$

The interval analysis program verifies that (50) is no larger than $2(1-c) \ln 4-$ 0.292 , for all $c \in[.67,1)$.

\section{A.4.3 The Boundary Cases That Are Line Segments}

In each case, we rule out the existence of a point on the boundary where $f$ exceeds $2(1-c) \ln 4$. We will compute a function that is either the limit of $f$ as its parameters approach the boundary or an upper bound on this limit. This new function will be continuous, and any maximum of this function will occur either where its first derivative is 0 or at an endpoint of the line segment. Cases 1-8 above evaluate the endpoints, and the analysis that follows will focus on the points where the first derivative is 0 . 
Case 9: the boundary with $\alpha=0$, and $t=0$.

Using (39), we get

$$
\begin{aligned}
\lim _{\substack{\alpha \rightarrow 0 \\
t \rightarrow 0}} f(\alpha, r, t)= & \ln 4-c \ln 4+\ln 3-c(2-3 r) \ln 3+c(1-3 r) \ln 2 \\
& -c(1-3 r) \ln (1-3 r)-c 3 r \ln r+r 3 c \ln r \\
& +(1-r) 3 c \ln (1-r)+\lim _{\alpha \rightarrow 0}(\Upsilon(x)-\alpha \Upsilon(z)-(1-\alpha) \Upsilon(y)) \\
\leq & \ln 12-c \ln 18+c r \ln 27-c r \ln 8-c(1-3 r) \ln (1-3 r) \\
& +3 c(1-r) \ln (1-r)+\Upsilon(x)-\Upsilon(y)
\end{aligned}
$$

where $r$ is in the range $\left[0,1-\frac{2}{3 c}\right]$.

The derivative of (51) with respect to $r$ is

$$
3 c \ln 3-3 c \ln 2+3 c \ln (1-3 r)-3 c \ln (1-r)+3 c \ln y,
$$

and setting the derivative to 0 gives the equation

$$
y=\frac{2(1-r)}{3(1-3 r)} .
$$

The interval analysis program verifies that (51) is no larger than $2(1-c) \ln 4-$ .007 , at each point of $c \in[.67,1)$ and $r \in\left(0,1-\frac{2}{3 c}\right)$ where (52) holds. This result plus the results of Case 1 and Case 3 proves that for $c \in[.67,1)$, each point on this boundary is smaller that $2(1-c) \ln 4-0.005$.

Case 10: the boundary with $\alpha=0$, and $t=r$.

Using (39), we get

$$
\begin{aligned}
\lim _{\substack{\alpha \rightarrow 0 \\
t \rightarrow r}} f(\alpha, r, t)= & \ln 4-c \ln 4+\ln 3-c(2-2 r) \ln 3+c(1-r) \ln 2 \\
& -c(1-r) \ln (1-r)-c r \ln r+r 3 c \ln r+(1-r) 3 c \ln (1-r) \\
& +\lim _{\alpha \rightarrow 0}(\Upsilon(x)-\alpha \Upsilon(z)-(1-\alpha) \Upsilon(y)) \\
\leq & \ln 12-c \ln 18+c r \ln 9-c r \ln 2+2 c(1-r) \ln (1-r) \\
& +2 c r \ln r+\Upsilon(x)-\Upsilon(y)
\end{aligned}
$$

where $r$ is in the range $\left[0,1-\frac{2}{3 c}\right]$.

The derivative of (53) with respect to $r$ is

$$
c \ln 9-c \ln 2-2 c \ln (1-r)+2 c \ln r+3 c \ln y,
$$

and setting the derivative to 0 gives the equation

$$
y^{3}=\frac{2(1-r)^{2}}{9 r^{2}} .
$$

The interval analysis program verifies that (53) is no larger than $2(1-c) \ln 4-$ 0.423 , at each point of $c \in[.67,1)$ and $r \in\left(0,1-\frac{2}{3 c}\right)$ where (54) holds. This result plus the results of Case 1 and Case 4 proves that for $c \in[.67,1)$, each point on this boundary is smaller that $2(1-c) \ln 4-0.046$. 
Case 11: the boundary with $\alpha=0$, and $r=1-\frac{2(1-\alpha)}{3 c}$.

Applying (39), (43), and Observation 35, we get the following.

$$
\begin{aligned}
\lim _{\substack{\alpha \rightarrow 0 \\
r \rightarrow 1-\frac{2}{3 c}}} f(\alpha, r, t)= & \ln 4-c \ln 4+\ln 3-(-c+t c+2) \ln 3 \\
& +(-2 c+2+2 t c) \ln 2 \\
& -(-2 c+2+2 t c) \ln \left(-2+\frac{2}{c}+2 t\right) \\
& -(3 c-2-3 c t) \ln \left(1-\frac{2}{3 c}-t\right)-c t \ln t \\
& +(3 c-2) \ln \left(1-\frac{2}{3 c}\right) \\
& +2 \ln \frac{2}{3 c}+\lim _{\substack{\alpha \rightarrow 0 \\
(\Upsilon)}}(\Upsilon(x)-\alpha \Upsilon(z)-(1-\alpha) \Upsilon(y)) \\
\leq & 2 \ln 4-3 \ln 3-c \ln 4+c \ln 3-4 c t \ln 3 \\
& +(3 c-2) \ln (3 c-2)-2(1-c+t c) \ln (1-c+t c) \\
& -(3 c-2-3 t c) \ln (3 c-2-3 t c) \\
& -c t \ln t-(2+t) c \ln c,
\end{aligned}
$$

where $t$ is in the range $\left[0,1-\frac{2}{3 c}\right]$.

The derivative of (55) with respect to $t$ is

$$
-4 c \ln 3-2 c \ln (1-c+t c)+3 c \ln (3 c-2-3 t c)-c \ln t-c \ln c,
$$

and setting the derivative to 0 gives the equation

$$
81 t c(1-c+t c)^{2}=(3 c-2-3 t c)^{3} .
$$

The interval analysis program verifies that (55) is no larger than $2(1-c) \ln 4-$ 0.015 , at each point of $c \in[.67,1)$ and $t \in\left(0,1-\frac{2}{3 c}\right)$ where (56) holds. This result plus the results of Case 3 and Case 4 proves that for $c \in[.67,1)$, each point on this boundary is smaller that $2(1-c) \ln 4-0.005$. 
Case 12: the boundary with $\alpha=1$, and $t=\frac{3 r-1}{2}$.

From (42),

$$
\begin{aligned}
\lim _{\substack{\alpha \rightarrow 1 \\
t \rightarrow \frac{3 r-1}{2}}} f(\alpha, r, t)= & \ln 4-c \ln 4-c\left(2+\frac{3 r-1}{2}-3 r\right) \ln 3 \\
& -3 c\left(r-\frac{3 r-1}{2}\right) \ln \left(r-\frac{3 r-1}{2}\right)-c \frac{3 r-1}{2} \ln \frac{3 r-1}{2} \\
& +r 3 c \ln r+(1-r) 3 c \ln (1-r) \\
& +\lim _{\alpha \rightarrow 1}(\Upsilon(x)-\alpha \Upsilon(z)-(1-\alpha) \Upsilon(y)) \\
\leq & \ln 4-c \ln 2-\frac{3 c}{2}(1-r) \ln 3+\frac{3 c}{2}(1-r) \ln (1-r) \\
& +3 c r \ln r-\frac{c}{2}(3 r-1) \ln (3 r-1)+\Upsilon(x)-\Upsilon(z)
\end{aligned}
$$

where $r$ is in the range $\left[\frac{2}{3 c}, 1\right]$.

The derivative of (57) with respect to $r$ is

$$
\frac{3 c}{2} \ln 3-\frac{3 c}{2} \ln (1-r)+3 c \ln r-\frac{3 c}{2} \ln (3 r-1)-3 c \ln z,
$$

and setting the derivative to 0 gives the equation

$$
3 r^{2}=z^{2}(1-r)(3 r-1) .
$$

The interval analysis program verifies that (57) is no larger than $2(1-c) \ln 4-$ 0.015 , at each point of $c \in[.67,1)$ and $r \in\left(\frac{2}{3 c}, 1\right)$ where (58) holds. This result plus the results of Case 2 and Case 6 proves that for each $c \in[.67,1)$ there exists a positive constant $b=b(c)$ such that each point on this boundary is smaller that $2(1-c) \ln 4-b(c)$.

Case 13: the boundary with $\alpha=1$, and $t=r$.

From (42),

$$
\begin{aligned}
\lim _{\substack{\alpha \rightarrow 1 \\
t \rightarrow r}} f(\alpha, r, t)= & \ln 4-c \ln 4+-c(2-2 r) \ln 3+c(1-r) \ln 2 \\
& -c(1-r) \ln (1-r)-c r \ln r+r 3 c \ln r+(1-r) 3 c \ln (1-r) \\
& +\lim _{\alpha \rightarrow 1}(\Upsilon(x)-\alpha \Upsilon(z)-(1-\alpha) \Upsilon(y)) \\
\leq & \ln 4-c \ln 2-c r \ln 2-c(1-r) \ln 9+2 c(1-r) \ln (1-r) \\
& +2 c r \ln r+\Upsilon(x)-\Upsilon(z)
\end{aligned}
$$

where $r$ is in the range $\left[\frac{2}{3 c}, 1\right]$.

The derivative of (59) with respect to $r$ is

$$
-c \ln 2+c \ln 9-2 c \ln (1-r)+2 c \ln r-3 c \ln z,
$$


and setting the derivative to 0 gives the equation

$$
9 r^{2}=2 z^{3}(1-r)^{2} .
$$

The interval analysis program verifies that (59) is no larger than $2(1-c) \ln 4-$ 0.015 , at each point of $c \in[.67,1)$ and $r \in\left(\frac{2}{3 c}, 1\right)$ where (60) holds. This result plus the results of Case 2 and Case 5 proves that for each $c \in[.67,1)$ there exists a positive constant $b=b(c)$ such that each point on this boundary is smaller that $2(1-c) \ln 4-b(c)$.

Case 14: the boundary with $\alpha=1$, and $r=\frac{2 \alpha}{3 c}$.

Applying (42), (40), and Observation 35, we get the following.

$$
\begin{aligned}
\lim _{\substack{\alpha \rightarrow 0 \\
r \rightarrow \frac{2}{3 c}}} f(\alpha, r, t)= & \ln 4-c \ln 4-(2 c+t c-2) \ln 3+(c-2+2 t c) \ln 2 \\
& -(c-2+2 t c) \ln \left(1-\frac{2}{c}+2 t\right)-(2-3 t c) \ln \left(\frac{2}{3 c}-t\right) \\
& -c t \ln t+2 \ln \frac{2}{3 c}+(3 c-2) \ln \left(1-\frac{2}{3 c}\right) \\
& +\lim _{\substack{\alpha \rightarrow 1 \\
r \rightarrow \frac{2}{3 c}}}(\Upsilon(x)-\alpha \Upsilon(z)-(1-\alpha) \Upsilon(y)) \\
\leq & \ln 4+4 \ln 3-c \ln 2-5 c \ln 3+c t \ln 4-2 c t \ln 9 \\
& +(3 c-2) \ln (3 c-2)-(c-2+2 t c) \ln (c-2+2 t c) \\
& -(2-3 t c) \ln (2-3 t c)-c t \ln t-(2+t) c \ln c
\end{aligned}
$$

where $t$ is in the range $\left[\frac{2-c}{2 c}, \frac{2}{3 c}\right]$.

The derivative of (61) with respect to $t$ is

$$
c \ln 4-2 c \ln 9-2 c \ln (c-2+2 t c)+3 c \ln (2-3 t c)-c \ln t-c \ln c,
$$

and setting the derivative to 0 gives the equation

$$
4(2-3 t c)^{3}=81 t c(c-2+2 t c)^{2} .
$$

The interval analysis program verifies that (61) is no larger than $2(1-c) \ln 4-$ 0.220 , at each point of $c \in[.67,1)$ and $t \in\left(\frac{2-c}{2 c}, \frac{2}{3 c}\right)$ where (62) holds. This result plus the results of Case 5 and Case 6 proves that for $c \in[.67,1)$, each point on this boundary is smaller that $2(1-c) \ln 4-0.071$. 
Case 15: the boundary with $r=\frac{2 \alpha}{3 c}$, and $t=r$.

Applying (40), we get the following.

$$
\begin{aligned}
\lim _{\substack{r \rightarrow \frac{2 \alpha}{3 c} \\
t \rightarrow r}} f(\alpha, r, t)= & \ln 4-c \ln 4+(1-\alpha) \ln 3-\left(2 c-\frac{4 \alpha}{3}\right) \ln 3 \\
& +\left(c-\frac{2 \alpha}{3}\right) \ln 2-\alpha \ln \alpha-(1-\alpha) \ln (1-\alpha) \\
& -\left(c-\frac{2 \alpha}{3}\right) \ln \left(1-\frac{2 \alpha}{3 c}\right)-\frac{2 \alpha}{3} \ln \frac{2 \alpha}{3 c}+2 \alpha \ln \frac{2 \alpha}{3 c} \\
& +(3 c-2 \alpha) \ln \left(1-\frac{2 \alpha}{3 c}\right) \\
& +\lim _{r \rightarrow \frac{2 \alpha}{3 c}}(\Upsilon(x)-\alpha \Upsilon(z)-(1-\alpha) \Upsilon(y)) \\
= & \ln 12-c \ln 162+\frac{\alpha}{3} \ln 12+\frac{a}{3} \ln \alpha-(1-\alpha) \ln (1-\alpha) \\
& +\frac{2}{3}(3 c-2 \alpha) \ln (3 c-2 \alpha)-2 c \ln c \\
& +\Upsilon(x)-(1-\alpha) \Upsilon(y)-\alpha \ln 2
\end{aligned}
$$

where $\alpha$ is in the range $[0,1]$.

The derivative of (63) with respect to $\alpha$ is

$$
\frac{1}{3} \ln 12+\frac{1}{3} \ln \alpha+\ln (1-\alpha)-\frac{4}{3} \ln (3 c-2 \alpha)+2 \ln y-\ln \left(\mathrm{e}^{y}-1-y\right)-\ln 2,
$$

and setting the derivative to 0 gives the equation

$$
3 \alpha(1-\alpha)^{3}=2(3 c-2 \alpha)^{4}\left(\frac{\mathrm{e}^{y}-1-y}{y^{2}}\right)^{3} .
$$

The interval analysis program verifies that (63) is no larger than $2(1-c) \ln 4-$ $7 \times 10^{-5}$, at each point of $c \in[.67,1)$ and $\alpha \in(0,1)$ where (64) holds. This result plus the results of Case 1 and Case 5 proves that for $c \in[.67,1)$, each point on this boundary is smaller that $2(1-c) \ln 4-7 \times 10^{-5}$. 
Case 16: the boundary with $r=\frac{2 \alpha}{3 c}$, and $t=\frac{3 r-1}{2}$.

Applying (40), we get

$$
\begin{aligned}
\lim _{\substack{r \rightarrow \frac{2 \alpha}{3 c} \\
t \rightarrow \frac{3 r-1}{2}}} f(\alpha, r, t)= & \ln 4-c \ln 4+(1-\alpha) \ln 3-\left(2 c+\frac{2 \alpha-c}{2}-2 \alpha\right) \ln 3 \\
& -\alpha \ln \alpha-(1-\alpha) \ln (1-\alpha) \\
& -3\left(\frac{2 \alpha}{3}-\frac{2 \alpha-c}{2}\right) \ln \left(\frac{2 \alpha}{3 c}-\frac{2 \alpha-c}{2 c}\right) \\
& -\frac{2 \alpha-c}{2} \ln \frac{2 \alpha-c}{2 c}+2 \alpha \ln \frac{2 \alpha}{3 c}+(3 c-2 \alpha) \ln \left(1-\frac{2 \alpha}{3 c}\right) \\
& +\lim _{r \rightarrow \frac{2 \alpha}{3 c}}(\Upsilon(x)-\alpha \Upsilon(z)-(1-\alpha) \Upsilon(y)) \\
= & \ln 12-c \ln 2-3 c \ln 3-\alpha \ln 3+2 \alpha \ln 2+\alpha \ln \alpha \\
& -(1-\alpha) \ln (1-\alpha)+\frac{3 c-2 \alpha}{2} \ln (3 c-2 \alpha) \\
& -\frac{2 \alpha-c}{2} \ln (2 \alpha-c) \\
& -2 c \ln c+\Upsilon(x)-(1-\alpha) \Upsilon(y)-\alpha \ln 2
\end{aligned}
$$

where $\alpha$ is in the range $\left[\frac{c}{2}, 1\right]$.

The derivative of (65) with respect to $\alpha$ is

$-\ln 3+\ln 4+\ln (1-\alpha)+\ln \alpha-\ln (3 c-2 \alpha)-\ln (2 \alpha-c)+2 \ln y-\ln \left(\mathrm{e}^{y}-1-y\right)-\ln 2$, and setting the derivative to 0 gives the equation

$$
2 \alpha(1-\alpha)=3(3 c-2 \alpha)(2 \alpha-c)\left(\frac{\mathrm{e}^{y}-1-y}{y^{2}}\right) .
$$

The interval analysis program verifies that (65) is no larger than $2(1-c) \ln 4-$ 0.012 , at each point of $c \in[.67,1)$ and $\alpha \in\left(\frac{c}{2}, 1\right)$ where (66) holds. This result plus the results of Case 6 and Case 7 proves that for $c \in[.67,1)$, each point on this boundary is smaller that $2(1-c) \ln 4-0.012$. 
Case 17: the boundary with $r=\frac{2 \alpha}{3 c}$, and $t=0$.

Applying (40), we get

$$
\begin{aligned}
\lim _{\substack{r \rightarrow \frac{2 \alpha}{3 c} \\
t \rightarrow 0}} f(\alpha, r, t)= & \ln 4-c \ln 4+(1-\alpha) \ln 3-(2 c-2 \alpha) \ln 3+(c-2 \alpha) \ln 2 \\
& -\alpha \ln \alpha-(1-\alpha) \ln (1-\alpha)-(c-2 \alpha) \ln \left(1-\frac{2 \alpha}{c}\right) \\
& -2 \alpha \ln \frac{2 \alpha}{3 c}+2 \alpha \ln \frac{2 \alpha}{3 c}+(3 c-2 \alpha) \ln \left(1-\frac{2 \alpha}{3 c}\right) \\
& +\lim _{r \rightarrow \frac{2 \alpha}{3 c}}(\Upsilon(x)-\alpha \Upsilon(z)-(1-\alpha) \Upsilon(y)) \\
= & \ln 12-c \ln 2-5 c \ln 3+3 \alpha \ln 3-\alpha \ln 4-(1-\alpha) \ln (1-\alpha) \\
& -\alpha \ln \alpha-(c-2 \alpha) \ln (c-2 \alpha)+(3 c-2 \alpha) \ln (3 c-2 \alpha) \\
& -2 c \ln c+\Upsilon(x)-(1-\alpha) \Upsilon(y)-\alpha \ln 2
\end{aligned}
$$

where $\alpha$ is in the range $\left[0, \frac{c}{2}\right]$.

The derivative of (67) with respect to $\alpha$ is

$3 \ln 3-\ln 4+\ln (1-\alpha)-\ln \alpha+2 \ln (c-2 \alpha)-2 \ln (3 c-2 \alpha)+2 \ln y-\ln \left(\mathrm{e}^{y}-1-y\right)-\ln 2$, and setting the derivative to 0 gives the equation

$$
27(1-\alpha)(c-2 \alpha)^{2}=8 \alpha(3 c-2 \alpha)^{2}\left(\frac{\mathrm{e}^{y}-1-y}{y^{2}}\right) .
$$

The interval analysis program verifies that (67) is no larger than $2(1-c) \ln 4-$ $2 \times 10^{-4}$, at each point of $c \in[.67,1)$ and $\alpha \in\left(0, \frac{c}{2}\right)$ where (68) holds. This result plus the results of Case 1 and Case 7 proves that for $c \in[.67,1)$, each point on this boundary is smaller that $2(1-c) \ln 4-2 \times 10^{-4}$. 
Case 18: the boundary with $r=1-\frac{2(1-\alpha)}{3 c}$, and $t=r$.

From (43), we have

$$
\begin{aligned}
\lim _{\substack{r \rightarrow 1-\frac{2 \alpha}{3 c} \\
t \rightarrow r}} f(\alpha, r, t)= & \ln 4-c \ln 4+(1-\alpha) \ln 3-\frac{4(1-\alpha)}{3} \ln 3+\frac{2(1-\alpha)}{3} \ln 2 \\
& -\alpha \ln \alpha-(1-\alpha) \ln (1-\alpha)-\frac{2(1-\alpha)}{3} \ln \frac{2(1-\alpha)}{3 c} \\
& -\left(c-\frac{2(1-\alpha)}{3}\right) \ln \left(1-\frac{2(1-\alpha)}{3 c}\right) \\
& +(3 c-2(1-\alpha)) 3 c \ln \left(1-\frac{2(1-\alpha)}{3 c}\right) \\
& +2(1-\alpha) \ln \frac{2(1-\alpha)}{3 c} \\
& +\lim _{r \rightarrow 1-\frac{2 \alpha}{3 c}}(\Upsilon(x)-\alpha \Upsilon(z)-(1-\alpha) \Upsilon(y)) \\
= & \ln 4-c \ln 36+2(1-\alpha) \ln 2-\frac{1-\alpha}{3} \ln 3+\frac{1-\alpha}{3} \ln (1-\alpha) \\
& -\alpha \ln \alpha+\frac{2}{3}(2 \alpha+3 c-2) \ln (2 \alpha+3 c-2)-2 c \ln c \\
& +\Upsilon(x)-\alpha \Upsilon(z)-(1-\alpha) \ln 2
\end{aligned}
$$

where $\alpha$ is in the range $[0,1]$.

The derivative of (69), with respect to $\alpha$ is

$-\ln 4+\frac{1}{3} \ln 3-\frac{1}{3} \ln (1-\alpha)-\ln \alpha+\frac{4}{3} \ln (2 \alpha+3 c-2)-2 \ln z+\ln \left(\mathrm{e}^{z}-1-z\right)+\ln 2$,

and setting the derivative to 0 gives the equation

$$
8(1-\alpha) \alpha^{3}=3(2 \alpha+3 c-2)^{4}\left(\frac{\mathrm{e}^{z}-1-z}{z^{2}}\right)^{3} .
$$

The interval analysis program verifies that (69) is no larger than $2(1-c) \ln 4-$ $3 \times 10^{-6}$, at each point of $c \in[.67,1)$ and $\alpha \in(0,1)$ where (70) holds. This result plus the results of Case 2 and Case 4 proves that for each $c \in[.67,1)$ there exists a positive constant $b=b(c)$ such that each point on this boundary is smaller that $2(1-c) \ln 4-b(c)$. 
Case 19: the boundary with $r=1-\frac{2(1-\alpha)}{3 c}$, and $t=\frac{3 r-1}{2}$.

From (43), we have

$$
\begin{aligned}
\lim _{\substack{r \rightarrow 1-\frac{2 \alpha}{3 c} \\
t \rightarrow \frac{3 r-1}{2}}} f(\alpha, r, t)= & \ln 4-c \ln 4+(1-\alpha) \ln 3-(1-\alpha) \ln 3-\alpha \ln \alpha \\
& -(1-\alpha) \ln (1-\alpha)-(1-\alpha) \ln \frac{1-\alpha}{3 c} \\
& -(c-(1-\alpha)) \ln \left(1-\frac{(1-\alpha)}{c}\right) \\
& +(3 c-2(1-\alpha)) \ln \left(1-\frac{2(1-\alpha)}{3 c}\right) \\
& +2(1-\alpha) \ln \frac{2(1-\alpha)}{3 c} \\
& +\lim _{r \rightarrow 1-\frac{2 \alpha}{3 c}}(\Upsilon(x)-\alpha \Upsilon(z)-(1-\alpha) \Upsilon(y)) \\
= & \ln 4-2(\alpha+c-1) \ln 2-(\alpha+3 c-1) \ln 3-\alpha \ln \alpha \\
& -(c+\alpha-1) \ln (c+\alpha-1) \\
& +(3 c-2+2 \alpha) \ln (3 c-2+2 \alpha)-2 c \ln c \\
& +\Upsilon(x)-\alpha \Upsilon(z)-(1-\alpha) \ln 2
\end{aligned}
$$

where $\alpha$ is in the range $[1-c, 1]$.

The derivative of (72) with respect to $\alpha$ is

$-\ln 3-2 \ln 2-\ln \alpha-\ln (c+\alpha-1)+2 \ln (3 c-2+2 \alpha)-2 \ln z+\ln \left(\mathrm{e}^{z}-1-z\right)+\ln 2$,

and setting the derivative to 0 gives the equation

$$
6 \alpha(c+\alpha-1)=(3 c-2+2 \alpha)^{2}\left(\frac{\mathrm{e}^{z}-1-z}{z^{2}}\right) .
$$

The interval analysis program verifies that (72) is no larger than $2(1-c) \ln 4-$ 0.034 , at each point of $c \in[.67,1)$ and $\alpha \in(1-c, 1)$ where (73) holds. This result plus the results of Case 2 and Case 8 proves that for each $c \in[.67,1)$ there exists a positive constant $b=b(c)$ such that each point on this boundary is smaller that $2(1-c) \ln 4-b(c)$. 
Case 20: the boundary with $r=1-\frac{2(1-\alpha)}{3 c}$, and $t=0$.

From (43), we have

$$
\begin{aligned}
\lim _{\substack{r \rightarrow 1-\frac{2 \alpha}{3 c} \\
t \rightarrow 0}} f(\alpha, r, t)= & \ln 4-c \ln 4+(1-\alpha) \ln 3-(-c+2(1-\alpha)) \ln 3 \\
& +(-2 c+2(1-\alpha)) \ln 2-\alpha \ln \alpha-(1-\alpha) \ln (1-\alpha) \\
& -(-2 c+2(1-\alpha)) \ln \left(-2+\frac{2(1-\alpha)}{c}\right) \\
& -(3 c-2(1-\alpha)) \ln \left(1-\frac{2(1-\alpha)}{3 c}\right) \\
& +(3 c-2(1-\alpha)) \ln \left(1-\frac{2(1-\alpha)}{3 c}\right) \\
& +2(1-\alpha) \ln \frac{2(1-\alpha)}{3 c} \\
& +\lim _{r \rightarrow 1-\frac{2 \alpha}{3 c}}(\Upsilon(x)-\alpha \Upsilon(z)-(1-\alpha) \Upsilon(y)) \\
= & \ln 16-\ln 27-c \ln 4+c \ln 3-\alpha \ln 4+\alpha \ln 27-\alpha \ln \alpha \\
& +(1-\alpha) \ln (1-\alpha)-2(1-\alpha-c) \ln (1-\alpha-c)-2 c \ln c \\
& +\Upsilon(x)-\alpha \Upsilon(z)-(1-\alpha) \ln 2
\end{aligned}
$$

where $\alpha$ is in the range $[0,1-c]$.

The derivative of (74) with respect to $\alpha$ is

$-\ln 4+\ln 27-\ln \alpha-\ln (1-\alpha)+2 \ln (1-\alpha-c)-2 \ln z+\ln \left(\mathrm{e}^{z}-1-z\right)+\ln 2$, and setting the derivative to 0 gives the equation

$$
2 \alpha(1-\alpha)=27(1-\alpha-c)^{2}\left(\frac{\mathrm{e}^{z}-1-z}{z^{2}}\right) .
$$

The interval analysis program verifies that (74) is no larger than $2(1-c) \ln 4-$ $9 \times 10^{-4}$, at each point of $c \in[.67,1)$ and $\alpha \in(0,1-c)$ where (75) holds. This result plus the results of Case 3 and Case 8 proves that for $c \in[.67,1)$, each point on this boundary is smaller that $2(1-c) \ln 4-9 \times 10^{-4}$.

Case 21: the boundary with $t=0=\frac{3 r-1}{2}$.

$$
\begin{aligned}
\lim _{\substack{r \rightarrow \frac{1}{3} \\
t \rightarrow 0}} f(\alpha, r, t)= & \ln 4-c \ln 4+(1-\alpha) \ln 3-c \ln 3-\alpha \ln \alpha \\
& -(1-\alpha) \ln (1-\alpha)-c \ln \frac{1}{3}+c \ln \frac{1}{3}+2 c \ln \frac{2}{3} \\
& +\lim _{r \rightarrow \frac{1}{3}}(\Upsilon(x)-\alpha \Upsilon(z)-(1-\alpha) \Upsilon(y)) \\
= & \ln 12-c \ln 27-\alpha \ln 3-\alpha \ln \alpha-(1-\alpha) \ln (1-\alpha) \\
& +\lim _{r \rightarrow \frac{1}{3}}(\Upsilon(x)-\alpha \Upsilon(z)-(1-\alpha) \Upsilon(y))
\end{aligned}
$$


where $\alpha$ is in the range $\left[1-c, \frac{c}{2}\right]$.

The derivative of (176) with respect to $\alpha$ is

$$
-\ln 3-\ln \alpha+\ln (1-\alpha)+\ln \left(\mathrm{e}^{z}-1-z\right)-\ln \left(\mathrm{e}^{y}-1-y\right),
$$

and setting the derivative to 0 gives the equation

$$
\frac{1-\alpha}{\alpha}=3 \frac{\mathrm{e}^{y}-1-y}{\mathrm{e}^{z}-1-z} .
$$

The interval analysis program verifies that (76) is no larger than $2(1-c) \ln 4-$ 0.130 , at each point of $c \in[.67,1)$ and $\alpha \in\left(1-c, \frac{c}{2}\right)$ where (77) holds. This result plus the results of Case 7 and Case 8 proves that for $c \in[.67,1)$, each point on this boundary is smaller that $2(1-c) \ln 4-0.130$.

\section{A.4.4 The Boundary Cases That Are Faces}

In each case, we rule out the existence of a point on the boundary where $f$ exceeds $2(1-c) \ln 4$. We will compute a function that is either the limit of $f$ as some parameter approaches the boundary or an upper bound on this limit. This new function will be continuous, and any maximum of this function will occur either where both its partial first derivatives are 0 or at an edge of the

face. Cases 9-21 above evaluate the line segments that form the boundaries of each face, and the analysis that follows will focus on the points on each face where both partial first derivatives are 0 .

Case 22: the boundary with $\alpha=0$.

Applying (39), we get

$$
\begin{aligned}
\lim _{\alpha \rightarrow 0} f(\alpha, r, t)= & \ln 4-c \ln 4+\ln 3-c(2+t-3 r) \ln 3+c(1-3 r+2 t) \ln 2 \\
& -c(1-3 r+2 t) \ln (1-3 r+2 t)-c(3 r-3 t) \ln (r-t) \\
& -c t \ln t+r 3 c \ln r+(1-r) 3 c \ln (1-r) \\
& +\lim _{\alpha \rightarrow 0}(\Upsilon(x)-\alpha \Upsilon(z)-(1-\alpha) \Upsilon(y)) \\
\leq & \ln 4-c \ln 4+\ln 3-c(2+t-3 r) \ln 3+c(1-3 r+2 t) \ln 2 \\
& -c(1-3 r+2 t) \ln (1-3 r+2 t)-c(3 r-3 t) \ln (r-t) \\
& -c t \ln t+r 3 c \ln r+(1-r) 3 c \ln (1-r) \\
& +\Upsilon(x)-\Upsilon(y)
\end{aligned}
$$

where $r$ is in the range $\left[0,1-\frac{2}{3 c}\right]$, and $t$ is in the range $[0, r]$. The partial derivative of (78) with respect to $r$ is

$3 c \ln 3-3 c \ln 2+3 c \ln (1-3 r+2 t)-3 c \ln (r-t)+3 c \ln r-3 c \ln (1-r)+3 c \ln y$, and the partial derivative with respect to $t$ is

$$
-c \ln 3+2 c \ln 2-2 c \ln (1-3 r+2 t)+3 c \ln (r-t)-c \ln t .
$$


Setting the derivatives to 0 give the equations

$$
\begin{aligned}
2(1-r)(r-t) & =3 y r(1-3 r+2 t) \\
3 t(1-3 r+2 t)^{2} & =4(r-t)^{3}
\end{aligned}
$$

which must hold for any assignment that maximizes (78). In addition, solving (79) for $t$ gives

$$
t=\frac{2(1-r) r-3 y r(1-3 r)}{2(1-r)+6 y r},
$$

and plugging this value into (800) and simplifying gives the equation

$$
9 r^{2} y^{3}=(2(1-r)-3 y(1-3 r))(1-r)
$$

which must also hold at any maximum of (78).

The interval analysis program verifies that (78) is no larger than $2(1-c) \ln 4-$ $5 \times 10^{-4}$, at each point of $c \in[.67,1), r \in\left(0,1-\frac{2}{3 c}\right)$, and $t \in(0, r)$ where (79), (80), and (81) all hold. This result plus the results of Cases 1, 3, 4, 9, 10, and 11 proves that for $c \in[.67,1)$, each point on this boundary is smaller that $2(1-c) \ln 4-5 \times 10^{-4}$.

Case 23: the boundary with $\alpha=1$.

Applying (42), we get

$$
\begin{aligned}
\lim _{\alpha \rightarrow 1} f(\alpha, r, t)= & \ln 4-c \ln 4-c(2+t-3 r) \ln 3+c(1-3 r+2 t) \ln 2 \\
& -c(1-3 r+2 t) \ln (1-3 r+2 t)-c(3 r-3 t) \ln (r-t) \\
& -c t \ln t+r 3 c \ln r+(1-r) 3 c \ln (1-r) \\
& +\lim _{\alpha \rightarrow 1}(\Upsilon(x)-\alpha \Upsilon(z)-(1-\alpha) \Upsilon(y)) \\
\leq & \ln 4-c \ln 4-c(2+t-3 r) \ln 3+c(1-3 r+2 t) \ln 2 \\
& -c(1-3 r+2 t) \ln (1-3 r+2 t)-c(3 r-3 t) \ln (r-t) \\
& -c t \ln t+r 3 c \ln r+(1-r) 3 c \ln (1-r) \\
& +\Upsilon(x)-\Upsilon(z)
\end{aligned}
$$

where $r$ is in the range $\left[\frac{2}{3 c}, 1\right]$, and $t$ is in the range $\left[\frac{3 r-1}{2}, r\right]$. The partial derivative of (82) with respect to $r$ is

$3 c \ln 3-3 c \ln 2+3 c \ln (1-3 r+2 t)-3 c \ln (r-t)+3 c \ln r-3 c \ln (1-r)-3 c \ln z$, and the partial derivative with respect to $t$ is

$$
-c \ln 3+2 c \ln 2-2 c \ln (1-3 r+2 t)+3 c \ln (r-t)-c \ln t .
$$

Setting the derivatives to 0 give the equations

$$
\begin{aligned}
& (1-r) 2 z(r-t)=3 r(1-3 r+2 t) \\
& 3 t(1-3 r+2 t)^{2}=4(r-t)^{3} .
\end{aligned}
$$


which must hold for any assignment that maximizes (82). In addition, solving (83) for $t$ gives

$$
t=\frac{2(1-r) r z-3 r(1-3 r)}{2(1-r) z+6 r},
$$

and plugging this value into (84) and simplifying gives the equation

$$
9 r^{2}=(2(1-r) z-3(1-3 r))(1-r) z^{2}
$$

which must also hold at any maximum of (82).

The interval analysis program verifies that (82) is no larger than $2(1-c) \ln 4-$ $5 \times 10^{-4}$, at each point of $c \in[.67,1), r \in\left(\frac{2}{3 c}, 1\right)$, and $t \in\left(\frac{3 r-1}{2}, r\right)$ where [83), (84), and (85) all hold. This result plus the results of Cases 2, 5, 6, 12, 13, and 14 proves that for each $c \in[.67,1)$ there exists a positive constant $b=b(c)$ such that each point on this boundary is smaller that $2(1-c) \ln 4-b(c)$.

\section{Case 24: the boundary with $r=\frac{2 \alpha}{3 c}$.}

Applying (40), we get

$$
\begin{aligned}
\lim _{r \rightarrow \frac{2 \alpha}{3 c}} f(\alpha, r, t)= & \ln 4-c \ln 4+(1-\alpha) \ln 3-(2 c+t c-2 \alpha) \ln 3 \\
& +(c-2 \alpha+2 t c) \ln 2-\alpha \ln \alpha-(1-\alpha) \ln (1-\alpha) \\
& -(c-2 \alpha+2 t c) \ln \left(1-\frac{2 \alpha}{c}+2 t\right) \\
& -(2 \alpha-3 t c) \ln \left(\frac{2 \alpha}{3 c}-t\right) \\
& -c t \ln t+2 \alpha \ln \frac{2 \alpha}{3 c}+(3 c-2 \alpha) \ln \left(1-\frac{2 \alpha}{3 c}\right) \\
& +\lim _{r \rightarrow \frac{2 \alpha}{3 c}}(\Upsilon(x)-\alpha \Upsilon(z)-(1-\alpha) \Upsilon(y)) \\
= & \ln 12-c \ln 18-c \ln 27+\alpha \ln 27+c t \ln 4-2 c t \ln 9 \\
& +\alpha \ln \alpha-(1-\alpha) \ln (1-\alpha)+(3 c-2 \alpha) \ln (3 c-2 \alpha) \\
& -(2 \alpha-3 t c) \ln (2 \alpha-3 t c)-t c \ln t \\
& -(c-2 \alpha+2 t c) \ln (c-2 \alpha+2 t c)-2 c \ln c-c t \ln c \\
& +\Upsilon(x)-\alpha \ln 2-(1-\alpha) \Upsilon(y)
\end{aligned}
$$

where $\alpha$ is in the range $[0,1]$, and $t$ is in the range $\left[\max \left\{0, \frac{2 \alpha-c}{2 c}\right\}, \frac{2 \alpha}{3 c}\right]$. The partial derivative of (86) with respect to $\alpha$ is

$$
\begin{aligned}
\ln 27+\ln \alpha+\ln (1-\alpha) & -2 \ln (3 c-2 \alpha)-2 \ln (2 \alpha-3 t c) \\
+ & 2 \ln (c-2 \alpha+2 t c)+2 \ln y-\ln \left(\mathrm{e}^{y}-1-y\right)-\ln 2,
\end{aligned}
$$

and the partial derivative with respect to $t$ is

$$
c \ln 4-2 c \ln 9+3 c \ln (2 \alpha-3 t c)-c \ln t-2 c \ln (c-2 \alpha+2 t c)-c \ln c .
$$


Setting the derivatives to 0 gives the equations

$$
\begin{aligned}
27 \alpha(1-\alpha)(c-2 \alpha+2 t c)^{2} & =2(3 c-2 \alpha)^{2}(2 \alpha-3 t c)^{2}\left(\frac{\mathrm{e}^{y}-1-y}{y^{2}}\right) \\
4(2 \alpha-3 t c)^{3} & =81 t c(c-2 \alpha+2 t c)^{2}
\end{aligned}
$$

which must hold at any maximum of (86).

The interval analysis program verifies that (86) is no larger than $2(1-c) \ln 4-$ $1 \times 10^{-9}$, at each point of $c \in[.67,1), \alpha \in(0,1)$, and $t \in\left(\max \left\{0, \frac{2 \alpha-c}{2 c}\right\}, \frac{2 \alpha}{3 c}\right)$ where (87) and (88) both hold. This result plus the results of Cases 1, 5, 6, 7, $14,15,16$, and 17 proves that for $c \in[.67,1)$, each point on this boundary is smaller that $2(1-c) \ln 4-1 \times 10^{-9}$.

Case 25: the boundary with $r=1-\frac{2(1-\alpha)}{3 c}$.

Applying (43), we get

$$
\begin{aligned}
\lim _{r \rightarrow 1-\frac{2(1-\alpha)}{3 c} f(\alpha, r, t)=} & \ln 4-c \ln 4+(1-\alpha) \ln 3-(-c+t c+2(1-\alpha)) \ln 3 \\
& +(-2 c+2(1-\alpha)+2 t c) \ln 2-\alpha \ln \alpha \\
& -(1-\alpha) \ln (1-\alpha) \\
& -(-2 c+2(1-\alpha)+2 t c) \ln \left(-2+\frac{2(1-\alpha)}{c}+2 t\right) \\
& -(3 c-2(1-\alpha)-3 t c) \ln \left(1-\frac{2(1-\alpha)}{3 c}-t\right) \\
& -c t \ln t+(3 c-2(1-\alpha)) \ln \left(1-\frac{2(1-\alpha)}{3 c}\right) \\
& +2(1-\alpha) \ln \frac{2(1-\alpha)}{3 c} \\
& +\lim (\Upsilon(x)-\alpha \Upsilon(z)-(1-\alpha) \Upsilon(y)) \\
& \ln 4-c \ln 108+(1-\alpha) \ln \frac{4}{27}+2 c(1-t) \ln 9-\alpha \ln \alpha \\
& +(1-\alpha) \ln (1-\alpha)+(3 c-2+2 \alpha) \ln (3 c-2+2 \alpha) \\
& -(3 c(1-t)-2+2 \alpha) \ln (3 c(1-t)-2+2 \alpha)-c t \ln t \\
& -2(1-\alpha-c(1-t)) \ln (1-\alpha-c(1-t)) \\
& -(2+t) c \ln c+\Upsilon(x)-\alpha \Upsilon(z)-(1-\alpha) \ln 2 \quad(89)
\end{aligned}
$$

where $\alpha$ is in the range $[0,1]$, and $t$ is in the range $\left[\min \left\{0, \frac{\alpha+c-1}{c}\right\}, 1-\frac{2(1-\alpha)}{3 c}\right]$. The partial derivative of (89) with respect to $\alpha$ is

$$
\begin{array}{r}
-\ln \frac{4}{27}-\ln \alpha-\ln (1-\alpha)+2 \ln (3 c-2+2 \alpha)-2 \ln (3 c(1-t)-2+2 \alpha) \\
+2 \ln (1-\alpha-c(1-t))-2 \ln z+\ln \left(\mathrm{e}^{z}-1-z\right)+\ln 2,
\end{array}
$$


and the partial derivative with respect to $t$ is

$$
-2 c \ln 9+3 c \ln (3 c(1-t)-2+2 \alpha)-c \ln t-2 c \ln (1-\alpha-c(1-t))-c \ln c .
$$

Setting the derivatives to 0 gives the equations

$$
\begin{aligned}
27(3 c-2+2 \alpha)^{2}(1-\alpha-c(1-t))^{2} & \left(\frac{\mathrm{e}^{z}-1-z}{z^{2}}\right) \\
& =2 \alpha(1-\alpha)(3 c(1-t)-2+2 \alpha)^{2} \\
(3 c(1-t)-2+2 \alpha)^{3} & =81 t c(1-\alpha-c(1-t))^{2}
\end{aligned}
$$

which must hold at any maximum of (89).

The interval analysis program verifies that (89) is no larger than $2(1-c) \ln 4-$ $7 \times 10^{-6}$, at each point of $c \in[.67,1), \alpha \in(0,1)$, and $t \in\left(\min \left\{0, \frac{\alpha+c-1}{c}\right\}, 1-\frac{2(1-\alpha)}{3 c}\right)$ where (91) and (92) both hold. This result plus the results of Cases 2, 3, 4, 8, $11,18,19$, and 20 proves that for each $c \in[.67,1)$ there exists a positive constant $b=b(c)$ such that each point on this boundary is smaller that $2(1-c) \ln 4-b(c)$.

Case 26: the boundary with $t=0$.

$$
\begin{aligned}
\lim _{t \rightarrow 0} f(\alpha, r, t)= & \ln 4-c \ln 4+(1-\alpha) \ln 3-c(2-3 r) \ln 3+c(1-3 r) \ln 2 \\
& -\alpha \ln \alpha-(1-\alpha) \ln (1-\alpha)-c(1-3 r) \ln (1-3 r) \\
& -3 c r \ln r+r 3 c \ln r+(1-r) 3 c \ln (1-r) \\
& +\Upsilon(x)-\alpha \Upsilon(z)-(1-\alpha) \Upsilon(y) \\
= & \ln 4-c \ln 18+(1-\alpha) \ln 3+3 r c \ln \frac{3}{2}-\alpha \ln \alpha \\
& -(1-\alpha) \ln (1-\alpha)-c(1-3 r) \ln (1-3 r) \\
& +(1-r) 3 c \ln (1-r)+\Upsilon(x)-\alpha \Upsilon(z)-(1-\alpha) \Upsilon(y)
\end{aligned}
$$

where $\alpha$ is in the range $\left[0, \frac{c}{2}\right]$ and $r$ is in the range $\left[\frac{2 \alpha}{3 c}, \min \left\{1-\frac{2(1-\alpha)}{3 c}, \frac{1}{3}\right\}\right]$. The partial derivative of (93) with respect to $\alpha$ is

$$
-\ln 3-\ln \alpha+\ln (1-\alpha)+\ln \left(\mathrm{e}^{z}-1-z\right)-\ln \left(\mathrm{e}^{y}-1-y\right),
$$

and with respect to $r$ is

$$
3 c \ln \frac{3}{2}+3 c \ln (1-3 r)-3 c \ln (1-r)-3 c \ln z+3 c \ln y .
$$

Setting the partial derivatives to 0 gives the equations

$$
\begin{aligned}
3 \alpha\left(\mathrm{e}^{y}-1-y\right) & =(1-\alpha)\left(\mathrm{e}^{z}-1-z\right) \\
2(1-r) z & =3(1-3 r) y
\end{aligned}
$$

which must hold at any maximum of (93). 
From (22),

$$
\frac{\mathrm{e}^{y}-1-y}{\mathrm{e}^{z}-1-z} \cdot \frac{1-r}{r}=\frac{1-\alpha}{a} \cdot \frac{y}{z} \cdot \frac{\mathrm{e}^{y}-1}{\mathrm{e}^{z}-1},
$$

and plugging in (94) gives

$$
\frac{1-r}{r}=3 \frac{y}{z} \cdot \frac{\mathrm{e}^{y}-1}{\mathrm{e}^{z}-1}
$$

Solving for $r$ in (95) gives

$$
r=\frac{3 y-2 z}{9 y-3 z}
$$

and thus

$$
\frac{1-r}{r}=\frac{6 y}{3 y-2 z}
$$

Combining (97) with (96) gives the equation

$$
2 z\left(\mathrm{e}^{z}-1\right)=(3 y-2 z)\left(\mathrm{e}^{z}-1\right)
$$

that we can use to further restrict the possible values $z$ and $y$ can have at a maximum for (93).

The interval analysis program verifies that (93) is no larger than $2(1-c) \ln 4-$ $7 \times 10^{-10}$, at each point of $c \in[.67,1), \alpha \in\left(0, \frac{c}{2}\right)$, and $r \in\left(\frac{2 \alpha}{3 c}, \min \left\{1-\frac{2(1-\alpha)}{3 c}, \frac{1}{3}\right\}\right)$ where (94), (95), and (98) all hold. This result plus the results of Cases 1, 3, 7, $8,9,17,20$, and 21 proves that for $c \in[.67,1)$, each point on this boundary is smaller that $2(1-c) \ln 4-7 \times 10^{-10}$.

Case 27: the boundary with $t=\frac{3 r-1}{2}$.

$$
\begin{aligned}
\lim _{t \rightarrow \frac{3 r-1}{2}} f(\alpha, r, t)= & \ln 4-c \ln 4+(1-\alpha) \ln 3-c\left(2+\frac{3 r-1}{2}-3 r\right) \ln 3 \\
& -\alpha \ln \alpha-(1-\alpha) \ln (1-\alpha) \\
& -3 c\left(r-\frac{3 r-1}{2}\right) \ln \left(r-\frac{3 r-1}{2}\right)-c \frac{3 r-1}{2} \ln \frac{3 r-1}{2} \\
& +r 3 c \ln r+(1-r) 3 c \ln (1-r) \\
& +\Upsilon(x)-\alpha \Upsilon(z)-(1-\alpha) \Upsilon(y) \\
= & \ln 4-c \ln 2+(1-\alpha) \ln 3-\frac{3}{2}(1-r) c \ln 3 \\
& -\alpha \ln \alpha-(1-\alpha) \ln (1-\alpha)-\frac{c}{2}(3 r-1) \ln (3 r-1) \\
& +3 c r \ln r+\frac{3}{2} c(1-r) \ln (1-r) \\
& +\Upsilon(x)-\alpha \Upsilon(z)-(1-\alpha) \Upsilon(y)
\end{aligned}
$$


where $\alpha$ is in the range $[1-c, 1]$ and $r$ is in the range $\left[\max \left\{\frac{2 \alpha}{3 c}, \frac{1}{3}\right\}, 1-\frac{2(1-\alpha)}{3 c}\right]$. The partial derivative of (99) with respect to $\alpha$ is

$$
-\ln 3-\ln \alpha+\ln (1-\alpha)+\ln \left(\mathrm{e}^{z}-1-z\right)-\ln \left(\mathrm{e}^{y}-1-y\right),
$$

and with respect to $r$ is

$$
\frac{3}{2} c \ln 3-\frac{3 c}{2} \ln (3 r-1)+3 c \ln r-\frac{3 c}{2} \ln (1-r)-3 c \ln z+3 c \ln y .
$$

Setting the partial derivatives to 0 gives the equations

$$
\begin{aligned}
3 \alpha\left(\mathrm{e}^{y}-1-y\right) & =(1-\alpha)\left(\mathrm{e}^{z}-1-z\right) \\
3 r^{2} y^{2} & =(3 r-1)(1-r) z^{2}
\end{aligned}
$$

which must hold at any maximum of (99).

From (22),

$$
\frac{\mathrm{e}^{y}-1-y}{\mathrm{e}^{z}-1-z} \cdot \frac{1-r}{r}=\frac{1-\alpha}{a} \cdot \frac{y}{z} \cdot \frac{\mathrm{e}^{y}-1}{\mathrm{e}^{z}-1},
$$

and plugging in (100) gives

$$
\frac{1-r}{r}=3 \frac{y}{z} \cdot \frac{\mathrm{e}^{y}-1}{\mathrm{e}^{z}-1}
$$

Solving for $r$ in (101) gives

$$
r=\frac{2 z^{2} \pm z \sqrt{z^{2}-3 y^{2}}}{3 z^{2}+3 y^{2}}
$$

and plugging this value into (102) gives two equations for the stationary points of (99):

$$
\begin{aligned}
& 3 \frac{y}{z} \cdot \frac{\mathrm{e}^{y}-1}{\mathrm{e}^{z}-1}=\frac{3 y^{2}+z^{2}-z \sqrt{z^{2}-3 y^{2}}}{2 z^{2}+z \sqrt{z^{2}-3 y^{2}}} \\
& 3 \frac{y}{z} \cdot \frac{\mathrm{e}^{y}-1}{\mathrm{e}^{z}-1}=\frac{3 y^{2}+z^{2}+z \sqrt{z^{2}-3 y^{2}}}{2 z^{2}-z \sqrt{z^{2}-3 y^{2}}} .
\end{aligned}
$$

We will use (103) and (104) in addition to the fact that $z^{2} \geq 3 y^{2}$ to restrict the values $y$ and $z$ can have at a maximum for (99).

However, note that if $y=0$, then any value of $z$ will satisfy (103). As a result, we need additional analysis to rule out cases where $y \in\left(0, y_{2}\right]$ and $z \in\left[z_{1}, z_{2}\right]$. Consider the two sides of (103).

$$
\begin{gathered}
\frac{3 y\left(\mathrm{e}^{y}-1\right)}{z\left(\mathrm{e}^{z}-1\right)} \\
\frac{3 y^{2}+z^{2}-z \sqrt{z^{2}-3 y^{2}}}{2 z^{2}+z \sqrt{z^{2}-3 y^{2}}} .
\end{gathered}
$$


The first derivatives of the two sides with respect to $y$ are

$$
\begin{gathered}
\frac{3\left(\mathrm{e}^{y}-1+y \mathrm{e}^{y}\right)}{z\left(\mathrm{e}^{z}-1\right)} \\
\frac{3 y}{z \sqrt{z^{2}-3 y^{2}}},
\end{gathered}
$$

and the second derivatives are

$$
\begin{gathered}
\frac{3 \mathrm{e}^{y}(y+2)}{z\left(\mathrm{e}^{z}-1\right)} \\
\frac{3 z}{\left(z^{2}-3 y^{2}\right)^{3 / 2}} .
\end{gathered}
$$

Note that both sides of (103) and their first derivatives are 0 when $y=0$, and both second derivatives are positive. As a result, if there is no $y \in\left(0, y_{1}\right]$ and $z \in\left[z_{1}, z_{2}\right]$ such that the two second derivatives are equal, then there is no $y \in\left(0, y_{1}\right]$ and $z \in\left[z_{1}, z_{3}\right]$ that satisfy (103).

The interval analysis program verifies that (99) is no larger than $2(1-c) \ln 4-$ $1 \times 10^{-7}$, at each point of $c \in[.67,1), \alpha \in(1-c, 1)$, and $r \in\left(\max \left\{\frac{2 \alpha}{3 c}, \frac{1}{3}\right\}, 1-\frac{2(1-\alpha)}{3 c}\right)$ where (100), (101), and $z^{2} \geq 3 y^{2}$ all hold and either (103) or (104) holds. In addition, if we are considering an interval for $y$ that has the lower bound equal to 0 , we use the additional test that (105) must equal (106) for some $y$ in that interval. This result plus the results of Cases $2,6,7,8,12,16,19$, and 21 proves that for each $c \in[.67,1)$ there exists a positive constant $b=b(c)$ such that each point on this boundary is smaller that $2(1-c) \ln 4-b(c)$.

Case 28: the boundary with $t=r$.

$$
\begin{aligned}
\lim _{t \rightarrow r} f(\alpha, r, t)= & \ln 4-c \ln 4+(1-\alpha) \ln 3-c(2-2 r) \ln 3 \\
& +c(1-r) \ln 2-\alpha \ln \alpha-(1-\alpha) \ln (1-\alpha) \\
& -c(1-r) \ln (1-r)-c r \ln r+r 3 c \ln r \\
& +(1-r) 3 c \ln (1-r) \\
& +\Upsilon(x)-\alpha \Upsilon(z)-(1-\alpha) \Upsilon(y) \\
= & \ln 4-c \ln 4+(1-\alpha) \ln 3-c(1-r) \ln \frac{9}{2} \\
& -\alpha \ln \alpha-(1-\alpha) \ln (1-\alpha) \\
& +2 c r \ln r+2 c(1-r) \ln (1-r) \\
& +\Upsilon(x)-\alpha \Upsilon(z)-(1-\alpha) \Upsilon(y)
\end{aligned}
$$

where $\alpha$ is in the range $[0,1]$ and $r$ is in the range $\left[\frac{2 \alpha}{3 c}, 1-\frac{2(1-\alpha)}{3 c}\right]$. The partial derivative of (99) with respect to $\alpha$ is

$$
-\ln 3-\ln \alpha+\ln (1-\alpha)+\ln \left(\mathrm{e}^{z}-1-z\right)-\ln \left(\mathrm{e}^{y}-1-y\right),
$$


and with respect to $r$ is

$$
c \ln \frac{9}{2}+2 c \ln r-2 c \ln (1-r)-3 c \ln z+3 c \ln y .
$$

Setting the partial derivatives to 0 gives the equations

$$
\begin{aligned}
3 \alpha\left(\mathrm{e}^{y}-1-y\right) & =(1-\alpha)\left(\mathrm{e}^{z}-1-z\right) \\
9 r^{2} y^{3} & =2(1-r)^{2} z^{3}
\end{aligned}
$$

which must hold at any maximum of (107).

From (22),

$$
\frac{\mathrm{e}^{y}-1-y}{\mathrm{e}^{z}-1-z} \cdot \frac{1-r}{r}=\frac{1-\alpha}{a} \cdot \frac{y}{z} \cdot \frac{\mathrm{e}^{y}-1}{\mathrm{e}^{z}-1},
$$

and plugging in (108) gives

$$
\frac{1-r}{r}=3 \frac{y}{z} \cdot \frac{\mathrm{e}^{y}-1}{\mathrm{e}^{z}-1}
$$

Rearranging (109) gives

$$
\frac{1-r}{r}=\sqrt{\frac{9 y^{3}}{2 z^{3}}}
$$

Combining (111) with (110) gives the equation

$$
\frac{\mathrm{e}^{z}-1}{\mathrm{e}^{y}-1}=\sqrt{\frac{2 z}{y}}
$$

that we can use to further restrict the possible values $z$ and $y$ can have at a maximum for (107).

In addition, we can see that (112) has exactly two non-negative solutions, one when $z=0$, and one when $z$ is between $\frac{y}{2}$ and $y$. As a result, we know that the positive solution has $z<y$. Applying $z<y$ to (22) gives $r<\alpha$, and applying $z<y$ to (108) gives $\alpha<\frac{1}{4}$. We can use this fact to further reduce the possible values that must hold at a maximum of (107).

The interval analysis program verifies that (107) is no larger than 2(1 -

c) $\ln 4-1 \times 10^{-4}$, at each point of $c \in[.67,1), \alpha \in(0,1)$, and $r \in\left(\frac{2 \alpha}{3 c}, 1-\frac{2(1-\alpha)}{3 c}\right)$ where (108), (109), (112), and $\alpha<\frac{1}{4}$ all hold. This result plus the results of Cases $1,2,4,5,10,13,15$, and 18 proves that for each $c \in[.67,1)$ there exists a positive constant $b=b(c)$ such that each point on this boundary is smaller that $2(1-c) \ln 4-b(c)$.

\section{A.5 The Interval Analysis Program}

The source code for the program is included with this paper on the arXiv.org site. The program uses and should be compiled with the Profil/BIAS [48] libraries for interval algorithms. The Profil/BIAS libraries are available at 
www.ti3.tu-harburg. de/Software/PROFILEnglisch.html.

For the program execution used to verify the cases of Lemma18, the program was compiled with version 2.0.8 of the Profil/BIAS libraries. The program was compiled and executed on an AMD Opteron 2350 processor running the Linux operating system, kernel version 2.6.27.56, and the program was compiled with the gcc compiler, version 4.3. 